\title{
Enantioselective Diels-Alder Reaction of Simple $\alpha, \beta$-Unsaturated Ketones with a Cinchona Alkaloid Catalyst
}

\author{
Ravi P. Singh, Keith Bartelson, Yi Wang, Heng Su, Xiaojie Lu and \\ Li Deng*
}

Department of Chemistry

Brandeis University

Waltham, Massachusetts 02454-9110

\section{Supporting Information}

General Information. ${ }^{1} \mathrm{H}$ and ${ }^{13} \mathrm{C}$ NMR spectra were recorded on a Varian instrument (400 $\mathrm{MHz}$ and $100 \mathrm{MHz}$, respectively) and internally referenced to tetramethylsilane signal or residual protio solvent signals. Data for ${ }^{1} \mathrm{H}$ NMR are recorded as follows: chemical shift ( $\delta$, ppm), multiplicity (s, singlet; d, doublet; t, triplet; $q$, quartet; quin, quintet; sext, sextet; m, multiplet), integration, coupling constant $(\mathrm{Hz})$. Data for ${ }^{13} \mathrm{C}$ NMR are reported in terms of chemical shift $(\delta, \mathrm{ppm})$. Infrared spectra were recorded on a Perkin Elmer FT-IR Spectrometer and are reported in frequency of absorption. Low resolution and high resolution mass spectra were recorded on either a Micromass 70-VSE-B instrument (EI, CI), Micromass Q-Tof instrument (ESI) or a Bruker Hct ultra Ptm Discovery System ${ }^{\mathrm{TM}}$. Specific rotations were measured on a Jasco Digital Polarimeter.

High performance liquid chromatography (HPLC) analysis was performed on a HewlettPackard 1100 Series instrument equipped with a quaternary pump, using a Daicel Chiralcel OJ, OD Column ( $250 \times 4.6 \mathrm{~mm})$ or Chiralpak AD, AS Column ( $250 \times 4.6 \mathrm{~mm})$. UV absorption was monitored at $215 \mathrm{~nm}$ or $220 \mathrm{~nm}$. 
Materials: 2-pyrone $5 a^{1}, 5 b^{2}, 5 c^{2}, 5 d^{3}$ and dienophile $6 B^{4}, 6 C^{4}, 6 D^{4}, 6 E^{4}, 6 F^{4}, 6 G^{4}$, $6 \mathbf{I}^{4}, 6 \mathbf{O}^{5}, 6 \mathrm{P}^{6}, 6 \mathrm{Q}^{7}$ were prepared according to literature methods. The cyclopentadiene, was purchased from Aldrich and used after cracking the dimmer. 1,3-cyclohexadiene and Dienophile 6A, 6H, 6N were purchased from Aldrich, $6 \mathbf{J}$ was purchased from ACROS, 6K, 6L, 6M were purchased from Alfa Aesar ${ }^{\circledR}$ and used without further purification.<smiles>O=c1occcc1O</smiles>

$5 a$<smiles>C=CC(=O)CC</smiles>

6J<smiles>O=c1occc(-c2ccccc2)c1O</smiles>

$5 b$<smiles>C=CC(=O)CCC</smiles>

$6 \mathrm{~K}$<smiles>Cc1ccoc(=O)c1O</smiles>

$5 \mathrm{c}$<smiles>O=c1occc(Cl)c1O</smiles>

5d<smiles>CCCCCC=CC(C)=O</smiles><smiles>C=CC(=O)CCCCC</smiles><smiles>CC=CC(C)=O</smiles>

$6 \mathrm{M}$

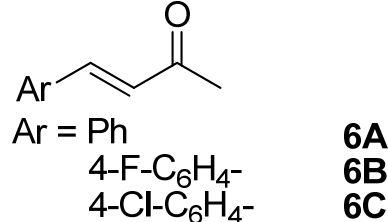

$4-\mathrm{Br}-\mathrm{C}_{6} \mathrm{H}_{4-}^{-} \quad$ 6D

4- $\mathrm{CF}_{3}-\mathrm{C}_{6} \mathrm{H}_{4-} \quad$ 6E 4-Me- $\mathrm{C}_{6} \mathrm{H}_{4-} \quad 6 \mathrm{~F}$ 4- $\mathrm{MeO}-\mathrm{C}_{6} \mathrm{H}_{4-}$ 6G 2-Thiophene- $\quad 6 \mathrm{H}$ 2-furyl-

$6 \mathrm{I}$<smiles>CC(=O)/C=C/CCCCl</smiles>

S-Figure 1. The Structure of 2-Pyrones 5 and Dienophiles 6

(For structure of Cinchona alkaloid catalysts, see S-Figure 2). Quinine and quinidine were purchased from Aldrich Inc. and used without further purification. Q-1 was prepared according to our reported method. ${ }^{8}$ Q-2, QD-4, Q-4 and QD-3 were prepared from commercially available quinine and quinidine following literature procedures. ${ }^{9,10}$<smiles>[R]OC(c1ccnc2ccc(O)cc12)C1C2CCN1C(C=C)C2</smiles>

Q-1 $\quad \mathrm{R}=\mathrm{PHN}$

( $\mathrm{PHN}: 5$ )<smiles>C=CC1C2CCN1[C@@H]([C@H](O)c1ccnc3ccc(OC)cc13)C2</smiles>

Quinine<smiles>C=CC1C2CCN1C([C@H](NC(=S)NC(Cl)Cl)c1ccnc3ccc(OC)cc13)C2</smiles>

Q-2

$$
\mathrm{Ar}=3,5-\text { bis } \mathrm{CF}_{3} \mathrm{Ph}-
$$<smiles>C=CC1CN2CCC1C2[C@H](N)c1ccnc2ccc(OC)cc12</smiles>

QD-4

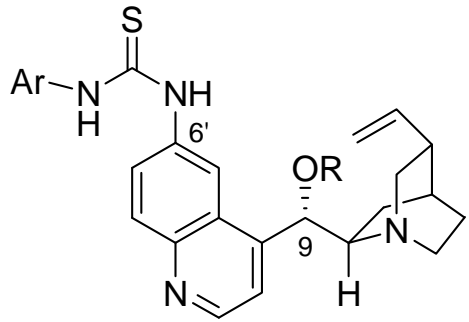

QD-3 $\mathrm{R}=\mathrm{PHN}$ $\mathrm{Ar}=3,5-$ bis $\mathrm{CF}_{3} \mathrm{Ph}-$<smiles>C=CC1CC2CCN1C2[C@H](N)c1ccnc2ccc(OC)cc12</smiles>

S-Figure 2. The Structures of Cinchona Alkaloids 


\section{Preparation of racemic Diels-Alder reaction adducts}

At room temperature to a solution of $\mathbf{3}(0.15 \mathrm{mmol})$ in methylene chloride $(0.30 \mathrm{~mL})$ was added 4 (2-4 equi.), and $\mathrm{DABCO}$ or $\mathrm{Et}_{3} \mathrm{~N}$ ( $20 \mathrm{~mol} \%$ ). After the reaction went to completion, the crude reaction mixture was purified by silica gel column chromatography and subsequently subjected to HPLC analysis.

\section{General reaction procedure for asymmetric Diels-Alder reaction}

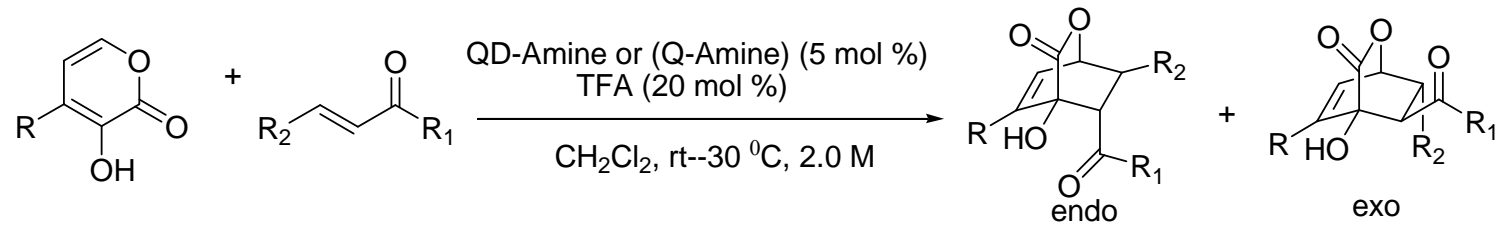

At room temperature, to a solution of 2-pyrones $5(0.25 \mathrm{mmol})$, catalyst QD-4 or Q-4 $(0.012 \mathrm{mmol}, 5 \mathrm{~mol} \%)$ in methylene chloride $(125 \mu \mathrm{L})$ and trifluroacetic acid $(3.8 \mu \mathrm{L}$, $0.05 \mathrm{mmol}, 20 \mathrm{~mol} \%)$ was added dienophiles 6 ( $0.5 \mathrm{mmol}, 2$ equiv.). The reaction mixture was kept at certain temperature for $72-120 \mathrm{~h}$ as specified in the following experimental section. The crude reaction mixture was passed through a short plug of silica gel for the removal of the catalyst, and the silica gel plug was washed with ethyl acetate (3.0-4.0 mL). The eluent was concentrated in vacuo, and the residue was subjected to silica gel flash chromatography.

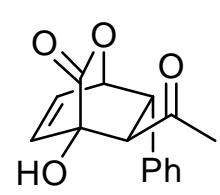

exo-7A

\section{8-acetyl-4-hydroxy-7-phenyl-2-oxa-bicyclo[2.2.2] oct-5-en-3-one (7A)}

exo-7A (major diastereomer) QD-4 catalyzed (4.0 mg, $0.012 \mathrm{mmol}, 5 \mathrm{~mol}$ $\%)$ reaction of $5 \mathbf{a}(28 \mathrm{mg}, 0.25 \mathrm{mmol})$ and $\mathbf{6 A}(73 \mathrm{mg}, 0.50 \mathrm{mmol}, 2.0$ equiv.) in the presence of trifluoroacetic acid $(3.8 \mu \mathrm{L}, 0.05 \mathrm{mmol}, 20 \mathrm{~mol}$ $\%)$ in methylene chloride $(125 \mu \mathrm{L})$ was run at $0{ }^{\circ} \mathrm{C}$ for $96 \mathrm{~h}$ to furnish the crude product \{exo:endo $=80: 20$, determined by integration of one set of ${ }^{1} \mathrm{H}$ NMR signals $\left[\delta_{\text {major }}=5.10\right.$ ppm (s), $\left.\left.\delta_{\text {minor }}=5.22 \mathrm{ppm}(\mathrm{d})\right]\right\}$. The crude product was purified by flash chromatography (silica gel, hexanes/ethyl acetate $=4 / 1$ ) to give pure exo-7A (major diastereomer) as a colorless solid ( $43.0 \mathrm{mg}, 69 \%$ yield) in $98 \%$ ee [determined by HPLC, Chiralcel OD, hexanes:isopropanol $=70: 30,1.0 \mathrm{~mL} / \mathrm{min}, \lambda=220 \mathrm{~nm}, \mathrm{t}$ (major) $=13.3$ $\min , \mathrm{t}($ minor $)=11.7 \mathrm{~min}][\alpha]^{\mathrm{D}}{ }_{25}=+120.4^{\circ}\left(c 1.03, \mathrm{CHCl}_{3}\right) ;{ }^{1} \mathrm{H} \mathrm{NMR}\left(400 \mathrm{MHz}, \mathrm{CDCl}_{3}\right)$ $\delta 2.20(\mathrm{~s}, 3 \mathrm{H}), 3.44(\mathrm{~d}, J=6.8 \mathrm{~Hz}, 1 \mathrm{H}), 3.73(\mathrm{dd}, J=3.6,6.8 \mathrm{~Hz}, 1 \mathrm{H}), 4.06(\mathrm{bs}, 1 \mathrm{H}), 5.10$ 
(s, 1H), $6.41(\mathrm{dd}, J=5.2,8.0 \mathrm{~Hz}, 1 \mathrm{H}), 6.68(\mathrm{~d}, J=8.0 \mathrm{~Hz}, 1 \mathrm{H}), 7.22(\mathrm{~d}, J=7.2 \mathrm{~Hz} 2 \mathrm{H})$, 7.27-7.37 (m, 3H); ${ }^{13} \mathrm{C}$ NMR (100 MHz, $\left.\mathrm{CDCl}_{3}\right) \delta 33.3,49.5,58.7,76.9,128.2,128.4$, 128.6, 129.3, 129.5, 137.9, 138.2, 173.5, 208.1; IR (neat) v 3691, 3054, 1764, 1717, 1422, 1263; HRMS (ESI/[M+Na] $]^{+}$) Calcd. for: $\mathrm{C}_{15} \mathrm{H}_{14} \mathrm{O}_{4} \mathrm{Na}: 281.0814$. Found: 281.0791.

Q-4 catalyzed reaction under the same conditions for $96 \mathrm{~h}$ furnished crude ent-7A (exo:endo $=82: 18$ ). After chromatographic purification, ent-exo-7A was obtained in 74\% yield $\{45.7 \mathrm{mg}, 96 \%$ ee, [determined by HPLC, Chiralcel OD, 70:30 Hexanes/Isopropanol, $1.0 \mathrm{~mL} / \mathrm{min}, \lambda=220 \mathrm{~nm}, \mathrm{t}$ (major) $=11.2 \mathrm{~min}, \mathrm{t}$ (minor) $=13.5$ $\left.\min ][\alpha]_{25}^{\mathrm{D}}=-133.4^{\circ}\left(c 1.01, \mathrm{CHCl}_{3}\right)\right\}$.

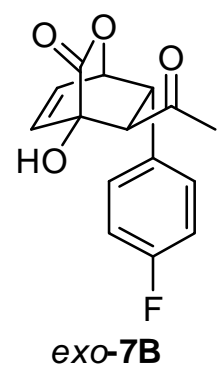

exo-7B

\section{8-acetyl-7-(4-fluorophenyl)-4-hydroxy-2-oxa-bicyclo[2.2.2]oct-5-en-3-} one (7B) exo-7B (major diastereomer)-QD-4 catalyzed (4.0 mg, 0.012 mmol, $5 \mathrm{~mol} \%)$ reaction of $5 \mathbf{a}(28 \mathrm{mg}, 0.25 \mathrm{mmol})$ and $\mathbf{6 B}(82 \mathrm{mg}, 0.50$ mmol, 2.0 equiv.) in the presence of trifluoroacetic acid (3.8 $\mu \mathrm{L}, 0.05$ mmol, $20 \mathrm{~mol} \%)$ in methylene chloride $(125 \mu \mathrm{L})$ was run at $0{ }^{\circ} \mathrm{C}$ for $96 \mathrm{~h}$ to furnish the crude product (exo:endo $=81: 19$, determined by integration

of one set of ${ }^{1} \mathrm{H}$ NMR signals $\left.\left[\delta_{\text {major }}=5.03 \mathrm{ppm}(\mathrm{s}), \delta_{\text {minor }}=5.16 \mathrm{ppm}(\mathrm{d})\right]\right\}$. The crude product was purified by flash chromatography ( silica gel, hexanes/ethyl acetate $=5 / 1$ ) to give pure exo-7B (major diastereomer) as a colorless solid (51.5 mg, 74\% yield) in 98\% ee [determined by HPLC, Chiralpak AD, hexanes:isopropanol $=70: 30,0.75 \mathrm{~mL} / \mathrm{min}, \lambda=$ $220 \mathrm{~nm}, \mathrm{t}$ (major) $=15.5 \mathrm{~min}, \mathrm{t}($ minor $)=12.1 \mathrm{~min}],[\alpha]^{\mathrm{D}}{ }_{25}=+121.5^{\circ}\left(c 1.3, \mathrm{CHCl}_{3}\right) ;{ }^{1} \mathrm{H}$ NMR (400 MHz, $\left.\mathrm{CDCl}_{3}\right) \delta 2.17(\mathrm{~s}, 3 \mathrm{H}), 3.33(\mathrm{~d}, J=6.8 \mathrm{~Hz}, 1 \mathrm{H}), 3.69$ (dd, $J=2.0,5.6$ Hz, 1H), 3.91 (bs, 1H), 5.03 (s, 1H), 6.37 (dd, $J=5.2,8.0 \mathrm{~Hz}, 1 \mathrm{H}), 6.65$ (d, $J=8.0 \mathrm{~Hz}$, $1 \mathrm{H}), 7.00(\mathrm{t}, J=8.4 \mathrm{~Hz}, 2 \mathrm{H}), 7.16(\mathrm{~m}, 2 \mathrm{H}) ;{ }^{13} \mathrm{C} \mathrm{NMR}\left(100 \mathrm{MHz}, \mathrm{CDCl}_{3}\right) \delta 33.3,48.7$, 58.9, 76.7, 76.8, 116.2, 116.4, 129.4, 129.7, 129.8, 138.5, 173.3, 207.7; IR (neat) v 3417, 1768, 1717, 1513, 1138; HRMS (ESI/[M+Na] ${ }^{+}$) Calcd. for: $\mathrm{C}_{15} \mathrm{H}_{13} \mathrm{FO}_{4} \mathrm{Na}: 299.0720$. Found: 299.0692.

\section{8-acetyl-7-(4-chlorophenyl)-4-hydroxy-2-oxa-bicyclo[2.2.2]oct-5-en-3-one (7C) exo-} 7C (major diastereomer)-QD-4 catalyzed (4.0 $\mathrm{mg}, 0.012 \mathrm{mmol}, 5 \mathrm{~mol} \%$ ) reaction of $5 \mathrm{a}$ (28 $\mathrm{mg}, 0.25 \mathrm{mmol})$ and $\mathbf{6 C}(90 \mathrm{mg}, 0.50 \mathrm{mmol}, 2.0$ equiv.) in the presence of trifluoroacetic acid $(3.8 \mu \mathrm{L}, 0.05 \mathrm{mmol}, 20 \mathrm{~mol} \%)$ in methylene chloride $(125 \mu \mathrm{L})$ was 


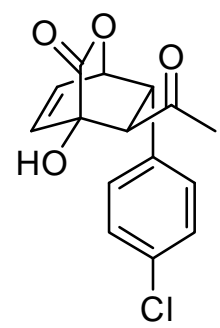

run at $0{ }^{\circ} \mathrm{C}$ for $96 \mathrm{~h}$ to furnish the crude product \{exo:endo $=79: 21$, determined by integration of one set of ${ }^{1} \mathrm{H}$ NMR signals $\left[\delta_{\text {major }}=5.04 \mathrm{ppm}\right.$ $\left.\left.(\mathrm{s}), \delta_{\text {minor }}=5.17 \mathrm{ppm}(\mathrm{d})\right]\right\}$. The crude product was purified by flash chromatography (silica gel, hexanes/ethyl acetate $=5 / 1$ ) to give pure exo7C (major diastereomer) as a colorless semisolid ( $50.4 \mathrm{mg}, 69 \%$ yield) in exo-7C 98\% ee [determined by HPLC, Chiralpak AD, hexanes:isopropanol $=$ $85: 15,1.0 \mathrm{~mL} / \mathrm{min}, \lambda=220 \mathrm{~nm}, \mathrm{t}$ (major) $=22.6 \mathrm{~min}, \mathrm{t}($ minor $)=17.8 \mathrm{~min}],[\alpha]_{25}^{\mathrm{D}}=$ $+112.3^{\circ}\left(\mathrm{c} 0.84, \mathrm{CHCl}_{3}\right) ;{ }^{1} \mathrm{H}$ NMR $\left(400 \mathrm{MHz}, \mathrm{CDCl}_{3}\right) \delta 2.18$ (s, 3H), 3.33 (d, J=6.8 Hz, $1 \mathrm{H}), 3.69(\mathrm{dd}, J=2.8,6.8 \mathrm{~Hz}, 1 \mathrm{H}), 3.86(\mathrm{bs}, 1 \mathrm{H}), 5.04(\mathrm{~d}, J=2.8 \mathrm{~Hz} 1 \mathrm{H}), 6.37(\mathrm{dd}, J=$ $4.8,8.0 \mathrm{~Hz}, 1 \mathrm{H}), 6.65(\mathrm{~d}, J=8.0 \mathrm{~Hz}, 1 \mathrm{H}), 7.12(\mathrm{~d}, J=8.8 \mathrm{~Hz}, 2 \mathrm{H}), 7.30$ (d, $J=8.0 \mathrm{~Hz}$, $2 \mathrm{H}) ;{ }^{13} \mathrm{C}$ NMR $\left(100 \mathrm{MHz}, \mathrm{CDCl}_{3}\right) \delta 33.4,48.8,58.7,76.6,76.7,129.3,129.4,129.5$, 134.5, 136.5 138.6, 173.2, 207.5; IR (neat) v 3412, 1762, 1712, 1494, 1140; HRMS $\left(\mathrm{ESI} /[\mathrm{M}+\mathrm{Na}]^{\dagger}\right.$ ) Calcd. for: $\mathrm{C}_{15} \mathrm{H}_{13} \mathrm{ClO}_{4} \mathrm{Na}: 315.0424$. Found: 315.0398.

Q-4 catalyzed reaction under the same conditions for $96 \mathrm{~h}$ furnished crude ent-7C (exo:endo $=79: 21)$. After chromatographic purification, ent-exo-7C was obtained in $74 \%$ yield $\{54 \mathrm{mg}, 96 \%$ ee, [determined by HPLC, Chiralpak AD, hexanes:isopropanol $=$ $85: 15,1.0 \mathrm{~mL} / \mathrm{min}, \lambda=220 \mathrm{~nm}, \mathrm{t}$ (major) $=17.9 \mathrm{~min}, \mathrm{t}($ minor $)=24.0 \mathrm{~min}][\alpha]_{25}^{\mathrm{D}}=-$ $142.1^{\circ}\left(\right.$ c $\left.\left.0.47, \mathrm{CHCl}_{3}\right)\right\}$.

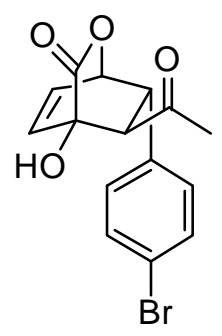

8-acetyl-7-(4-bromophenyl)-4-hydroxy-2-oxa-bicyclo[2.2.2]oct-5-en-3one (7D) exo-7D (major diastereomer)-QD-4 catalyzed $(4.0 \mathrm{mg}, 0.012$ mmol, $5 \mathrm{~mol} \%)$ reaction of $5 \mathbf{a}(28 \mathrm{mg}, 0.25 \mathrm{mmol})$ and $6 \mathbf{D}(112 \mathrm{mg}, 0.50$ mmol, 2.0 equiv.) in the presence of trifluoroacetic acid (3.8 $\mu \mathrm{L}, 0.05$ mmol, $20 \mathrm{~mol} \%)$ in methylene chloride $(125 \mu \mathrm{L})$ was run at $0{ }^{\circ} \mathrm{C}$ for $72 \mathrm{~h}$ exo-7D to furnish the crude product exo:endo $=81: 19$, determined by integration of one set of ${ }^{1} \mathrm{H}$ NMR signals $\left.\left[\delta_{\text {major }}=5.04 \mathrm{ppm}(\mathrm{s}), \delta_{\text {minor }}=5.17 \mathrm{ppm}(\mathrm{d})\right]\right\}$. The crude product was purified by flash chromatography (silica gel, hexanes/ethyl acetate $=5 / 1$ ) to give pure exo-7D (major diastereomer) as a colorless solid (64 mg, 76\% yield) in 97\% ee [determined by HPLC, Chiralpak AD, hexanes:isopropanol $=85: 15,1 \mathrm{~mL} / \mathrm{min}, \lambda=220$ $\mathrm{nm}, \mathrm{t}$ (major) $=16.9 \mathrm{~min}, \mathrm{t}$ (minor) $=13.8 \mathrm{~min}],[\alpha]^{\mathrm{D}}{ }_{25}=+132.3^{\circ}\left(\right.$ c $\left.1.25, \mathrm{CHCl}_{3}\right) ;{ }^{1} \mathrm{H}$ NMR (400 MHz, $\left.\mathrm{CDCl}_{3}\right) \delta 2.18(\mathrm{~s}, 3 \mathrm{H}), 3.33(\mathrm{~d}, J=6.8 \mathrm{~Hz}, 1 \mathrm{H}), 3.67$ (dd, $J=3.2,6.8$ 
$\mathrm{Hz}, 1 \mathrm{H}$ ), 3.90 (bs, 1H), 5.04 (quin, $J=2.4 \mathrm{~Hz} 1 \mathrm{H}$ ), 6.37 (dd, $J=5.2,8.0 \mathrm{~Hz}, 1 \mathrm{H}$ ), 6.65 $(\mathrm{dd}, J=2.0,8.0 \mathrm{~Hz}, 1 \mathrm{H}), 7.06(\mathrm{~d}, J=8.0 \mathrm{~Hz}, 2 \mathrm{H}), 7.45(\mathrm{~d}, J=8.8 \mathrm{~Hz}, 2 \mathrm{H}) ;{ }^{13} \mathrm{C} \mathrm{NMR}$ $\left(100 \mathrm{MHz}, \mathrm{CDCl}_{3}\right) \delta 33.4,48.9,58.6,76.5,76.7,122.5,129.3,129.8,132.5,137.0$, 138.6, 173.2, 207.6; IR (neat) $v$ 3412, 1762, 1712, 1494, 1140; HRMS (ESI/[M+Na] $\left.]^{+}\right)$ Calcd. for: $\mathrm{C}_{15} \mathrm{H}_{13} \mathrm{BrO}_{4} \mathrm{Na}: 358.9919$. Found: 358.9904 .

Q-4 catalyzed reaction under the same conditions for $72 \mathrm{~h}$ furnished crude ent-7D (exo:endo $=81: 19$ ). After chromatographic purification, ent-exo-7D was obtained in $74 \%$ yield $\{53.5 \mathrm{mg}, 97 \%$ ee, [determined by HPLC, Chiralpak AD, hexanes:isopropanol $=$ $85: 15,1.0 \mathrm{~mL} / \mathrm{min}, \lambda=220 \mathrm{~nm}, \mathrm{t}$ (major) $=13.8 \mathrm{~min}, \mathrm{t}($ minor $)=17.7 \mathrm{~min}][\alpha]_{25}^{\mathrm{D}}=-$ $139.4^{\circ}\left(\right.$ c $\left.\left.0.97, \mathrm{CHCl}_{3}\right)\right\}$.

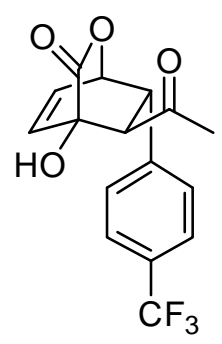

8-acetyl-4-hydroxy-7-(4-(trifluoromethyl)phenyl)-2-oxa-bicyclo[2.2.2] oct-5-en-3-one (7E) - exo-7E (major diastereomer)- QD-4 catalyzed (4.0 $\mathrm{mg}, 0.012 \mathrm{mmol}, 5 \mathrm{~mol} \%)$ reaction of $5 \mathrm{a}(28 \mathrm{mg}, 0.25 \mathrm{mmol})$ and $\mathbf{6 E}(107$ $\mathrm{mg}, 0.50 \mathrm{mmol}, 2.0$ equiv.) in the presence of trifluoroacetic acid $(3.8 \mu \mathrm{L}$, $0.05 \mathrm{mmol}, 20 \mathrm{~mol} \%)$ in methylene chloride $(125 \mu \mathrm{L})$ was run at $-20{ }^{\circ} \mathrm{C}$ exo-7E

for $96 \mathrm{~h}$ to furnish the crude product \{exo:endo $=84: 16$, determined by integration of one set of ${ }^{1} \mathrm{H}$ NMR signals $\left.\left[\delta_{\text {major }}=5.09 \mathrm{ppm}(\mathrm{d}), \delta_{\text {minor }}=5.22 \mathrm{ppm}(\mathrm{d})\right]\right\}$. The crude product was purified by flash chromatography (silica gel, hexanes/ethyl acetate $=5 / 1)$ to give pure exo-7E (major diastereomer) as a white solid $(66.0 \mathrm{mg}, 81 \%$ yield) in 97\% ee [determined by HPLC, Chiralpak AD, hexanes:isopropanol $=90: 10,1.0 \mathrm{~mL} / \mathrm{min}$, $\lambda=220 \mathrm{~nm}, \mathrm{t}$ (major) $=30.4 \mathrm{~min}, \mathrm{t}($ minor $)=22.3 \mathrm{~min}],[\alpha]_{25}^{\mathrm{D}}=+120.2^{\circ}\left(\mathrm{c} 1.0, \mathrm{CHCl}_{3}\right)$; ${ }^{1} \mathrm{H}$ NMR (400 MHz, $\left.\mathrm{CDCl}_{3}\right) \delta 2.20(\mathrm{~s}, 3 \mathrm{H}), 3.39(\mathrm{~d}, J=6.8 \mathrm{~Hz}, 1 \mathrm{H}), 3.81(\mathrm{dd}, J=2.8$, $6.0 \mathrm{~Hz}, 1 \mathrm{H}$ ), 3.88 (bs, 1H), 5.09 (d, $J=1.2 \mathrm{~Hz}, 1 \mathrm{H}), 6.39$ (dd, $J=5.6,7.2 \mathrm{~Hz}, 1 \mathrm{H}), 6.69$ (d, $J=7.2 \mathrm{~Hz}, 1 \mathrm{H}), 7.32$ (d, $J=8.0 \mathrm{~Hz}, 2 \mathrm{H}), 7.59$ (d, $J=8.0 \mathrm{~Hz}, 2 \mathrm{H}) ;{ }^{13} \mathrm{C}$ NMR $(100$ $\left.\mathrm{MHz}, \mathrm{CDCl}_{3}\right) \delta 33.5,49.2,58.4,76.4,76.7,126.3,128.6,129.3,138.8,142.0,173.1$, 207.4; IR (neat) $\vee 3407,1752,1713,1621,1414,1328,1149$; HRMS (ESI/[M+Na] $\left.]^{+}\right)$ Calcd. for: $\mathrm{C}_{16} \mathrm{H}_{13} \mathrm{~F}_{3} \mathrm{O}_{4} \mathrm{Na}: 349.0664$. Found: 349.0648 .

8-acetyl-4-hydroxy-7-p-tolyl-2-oxa-bicyclo[2.2.2]oct-5-en-3-one (7F) exo-7F (major diastereomer)- QD-4 catalyzed (4.0 mg, $0.012 \mathrm{mmol}, 5 \mathrm{~mol} \%$ ) reaction of $5 \mathbf{a}(28 \mathrm{mg}$, $0.25 \mathrm{mmol}$ ) and $\mathbf{6 F}$ ( $80 \mathrm{mg}, 0.50 \mathrm{mmol}, 2.0$ equiv.) in the presence of trifluoroacetic acid 
(3.8 $\mu \mathrm{L}, 0.05 \mathrm{mmol}, 20 \mathrm{~mol} \%)$ in methylene chloride $(125 \mu \mathrm{L})$ was run at $0{ }^{\circ} \mathrm{C}$ for $96 \mathrm{~h}$

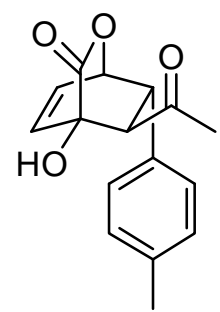

exo-7F to furnish the crude product $\{$ exo:endo $=80: 20$, determined by integration of one set of ${ }^{1} \mathrm{H}$ NMR signals $\left[\delta_{\text {major }}=5.03 \mathrm{ppm}(\mathrm{s}), \delta_{\text {minor }}=5.15 \mathrm{ppm}\right.$ (d)]\}. The crude product was purified by flash chromatography (silica gel, hexanes/ethyl acetate $=4 / 1$ ) to give pure exo-7F (major diastereomer) as a colorless semisolid (43 mg, 63\% yield) in 97\% ee [determined by HPLC, Chiralpak AD, hexanes:isopropanol $=80: 20,1.0 \mathrm{~mL} / \mathrm{min}, \lambda=220 \mathrm{~nm}, \mathrm{t}$ $($ major $)=15.4 \mathrm{~min}, \mathrm{t}($ minor $)=11.0 \mathrm{~min}],[\alpha]_{25}^{\mathrm{D}}=+143.5^{\circ}\left(\right.$ c $\left.1.20, \mathrm{CHCl}_{3}\right) ;{ }^{1} \mathrm{H} \mathrm{NMR}$ $\left(400 \mathrm{MHz}, \mathrm{CDCl}_{3}\right) \delta 2.15(\mathrm{~s}, 3 \mathrm{H}), 2.30(\mathrm{~s}, 3 \mathrm{H}), 3.36(\mathrm{~d}, J=3.33 \mathrm{~Hz}, 1 \mathrm{H}), 3.63$ (dd, $J=$ $3.2,6.8 \mathrm{~Hz}, 1 \mathrm{H}), 3.86(\mathrm{bs}, 1 \mathrm{H}), 5.03(\mathrm{~s}, 1 \mathrm{H}), 6.36(\mathrm{dd}, J=4.8,8.0 \mathrm{~Hz}, 1 \mathrm{H}), 6.62(\mathrm{~d}, J=$ $8.0 \mathrm{~Hz}, 1 \mathrm{H}), 7.05$ (d, $J=7.2 \mathrm{~Hz}, 2 \mathrm{H}), 7.12$ (d, $J=8.0 \mathrm{~Hz}, 2 \mathrm{H}) ;{ }^{13} \mathrm{C}$ NMR $(100 \mathrm{MHz}$, $\mathrm{CDCl} 3) \delta 21.2,33.2,49.2,58.8,76.7,77.1,128.0,128.5,129.5,129.9,134.9,138.1$, 138.3, 173.5, 208.0; IR (neat) $v$ 3444, 2922, 1763, 1713, 1359, 1139; HRMS (ESI/[M+Na $]^{+}$) Calcd. for: $\mathrm{C}_{16} \mathrm{H}_{16} \mathrm{O}_{4} \mathrm{Na}: 295.0970$. Found: 295.0948 .

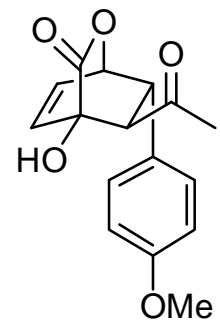

exo-7G

\section{8-acetyl-4-hydroxy-7-(4-methoxyphenyl)-2-oxa-bicyclo[2.2.2]oct-5-en-}

3-one (7G) exo-7G (major diastereomer)-QD-4 catalyzed (4.0 mg, 0.012 mmol, $5 \mathrm{~mol} \%)$ reaction of $5 \mathbf{a}(28 \mathrm{mg}, 0.25 \mathrm{mmol})$ and $\mathbf{6 G}(88 \mathrm{mg}, 0.50$ mmol, 2.0 equiv.) in the presence of trifluoroacetic acid $(3.8 \mu \mathrm{L}, 0.05$ mmol, $20 \mathrm{~mol} \%)$ in methylene chloride $(125 \mu \mathrm{L})$ was run at $0{ }^{\circ} \mathrm{C}$ for $96 \mathrm{~h}$ to furnish the crude product exo:endo $=84: 16$, determined by integration of one set of ${ }^{1} \mathrm{H}$ NMR signals $\left.\left[\delta_{\text {major }}=5.00 \mathrm{ppm}(\mathrm{s}), \delta_{\text {minor }}=5.13 \mathrm{ppm}(\mathrm{d})\right]\right\}$. The crude product was purified by flash chromatography (silica gel, hexanes/ethyl acetate $=5 / 1$ ) to give pure exo-7G (major diastereomer) as a colorless semisolid (41 mg, 57\% yield) in 97\% ee [determined by HPLC, Chiralpak AD, hexanes:isopropanol $=80: 20,0.75$ $\mathrm{mL} / \mathrm{min}, \lambda=220 \mathrm{~nm}, \mathrm{t}$ (major) $=27.4 \mathrm{~min}, \mathrm{t}$ (minor) $=19.7 \mathrm{~min}],[\alpha]^{\mathrm{D}}{ }_{25}=+150.7^{\circ}(\mathrm{c}$ 1.40, $\left.\mathrm{CHCl}_{3}\right) ;{ }^{1} \mathrm{H}$ NMR (400 MHz, $\left.\mathrm{CDCl}_{3}\right) \delta 2.14(\mathrm{~s}, 3 \mathrm{H}), 3.33(\mathrm{~d}, J=6.8 \mathrm{~Hz}, 1 \mathrm{H}), 3.61$ $(\mathrm{dd}, J=4.0,6.8 \mathrm{~Hz}, 1 \mathrm{H}), 3.76(\mathrm{~s}, 3 \mathrm{H}), 3.88(\mathrm{bs}, 1 \mathrm{H}), 5.00(\mathrm{~d}, J=2.0 \mathrm{~Hz}, 1 \mathrm{H}), 6.36(\mathrm{dd}$, $J=5.2,8.0 \mathrm{~Hz}, 1 \mathrm{H}), 6.62(\mathrm{~d}, J=7.6 \mathrm{~Hz}, 1 \mathrm{H}), 6.83(\mathrm{~d}, J=8.0 \mathrm{~Hz}, 2 \mathrm{H}), 7.09(\mathrm{~d}, J=8.0$ $\mathrm{Hz}, 2 \mathrm{H}) ;{ }^{13} \mathrm{C} \mathrm{NMR}\left(100 \mathrm{MHz}, \mathrm{CDCl}_{3}\right) \delta 33.2,48.8,55.5,59.0,76.7,77.2,114.6,129.2$, 129.5, 129.9, 138.2, 159.6, 173.5, 208.0; IR (neat) v 3446, 1762, 1710, 1514, 1252, 1140; HRMS (ESI/[M+Na] $]^{+}$) Calcd. for: $\mathrm{C}_{16} \mathrm{H}_{16} \mathrm{O}_{5} \mathrm{Na}: 311.0919$. Found: 311.0882. 


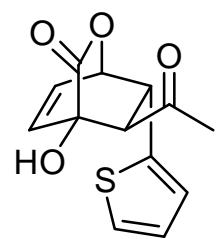

exo-7H

\section{8-acetyl-4-hydroxy-7-(thiophen-2-yl)-2-oxa-bicyclo[2.2.2]oct-5-en-3-}

one (7H)- exo-7H (major diasteromer)-QD-4 catalyzed (4.0 mg, 0.012 $\mathrm{mmol}, 5 \mathrm{~mol} \%)$ reaction of $\mathbf{5 a}(28 \mathrm{mg}, 0.25 \mathrm{mmol})$ and $\mathbf{6 H}(76 \mathrm{mg}, 0.50$ mmol, 2.0 equiv.) in the presence of trifluoroacetic acid $(3.8 \mu \mathrm{L}, 0.05$ mmol, $20 \mathrm{~mol} \%)$ in methylene chloride $(125 \mu \mathrm{L})$ was run at $0{ }^{\circ} \mathrm{C}$ for $96 \mathrm{~h}$

to furnish the crude product $\{$ exo:endo $=80: 20$, determined by integration of one set of

${ }^{1} \mathrm{H}$ NMR signals $\left.\left[\delta_{\text {major }}=5.14 \mathrm{ppm}(\mathrm{d}), \delta_{\text {minor }}=5.24 \mathrm{ppm}(\mathrm{d})\right]\right\}$. The crude product was purified by flash chromatography ( silica gel, hexanes/ethyl acetate $=4 / 1$ ) to give pure exo-7H (major diastereomer) as a white solid (45.0 $\mathrm{mg}, 68 \%$ yield) in $99 \%$ ee [determined by HPLC, Chiralpak AD + Chiralcel OJ, hexanes:isopropanol $=80: 20,1.0$ $\mathrm{mL} / \mathrm{min}, \lambda=220 \mathrm{~nm}, \mathrm{t}$ (major) $=55.8 \mathrm{~min}, \mathrm{t}($ minor $)=47.3 \mathrm{~min}],[\alpha]^{\mathrm{D}}{ }_{25}=+104.2^{\circ}(\mathrm{c}$ 0.95, $\left.\mathrm{CHCl}_{3}\right) ;{ }^{1} \mathrm{H} \mathrm{NMR}\left(400 \mathrm{MHz}, \mathrm{CDCl}_{3}\right) \delta 2.23(\mathrm{~d}, J=4.8 \mathrm{~Hz}, 3 \mathrm{H}), 3.30(\mathrm{t}, J=5.6 \mathrm{~Hz}$, $1 \mathrm{H}), 3.85$ (bs, 1H), 3.98 (d, $J=4.0 \mathrm{~Hz}, 1 \mathrm{H}), 5.12$ (d, $J=1.2 \mathrm{~Hz}, 1 \mathrm{H}), 6.46-6.42(\mathrm{~m}, 1 \mathrm{H})$, 6.63-6.61 (m, 1H), $6.86(\mathrm{~s}, 1 \mathrm{H}), 6.94-6.90(\mathrm{~m}, 1 \mathrm{H}), 7.23-7.18(\mathrm{~m}, 1 \mathrm{H}) ;{ }^{13} \mathrm{C}$ NMR $(100$ $\mathrm{MHz}, \mathrm{CDCl} 3) \delta 33.4,44.8,60.7,76.5,76.6,125.6,126.4,127.5,129.5,138.8,141.7$, 173.0, 207.5; IR (neat) $v$ 3452, 3105, 2924, 1764, 1713, 1624, 1358, 1140; HRMS $\left(\mathrm{ESI} /[\mathrm{M}+\mathrm{Na}]^{\dagger}\right.$ ) Calcd. for: $\mathrm{C}_{13} \mathrm{H}_{12} \mathrm{O}_{4} \mathrm{SNa}$ : 287.0378. Found: 287.0353.

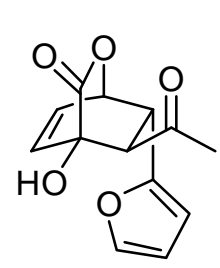

exo-7I

8-acetyl-7-(furan-2-yl)-4-hydroxy-2-oxa-bicyclo[2.2.2] oct-5-en-3-one (7I)-exo-7I (major diastereomer)-QD-4 catalyzed (4.0 mg, $0.012 \mathrm{mmol}, 5$ $\mathrm{mol} \%)$ reaction of $\mathbf{5 a}(28 \mathrm{mg}, 0.25 \mathrm{mmol})$ and $\mathbf{6 I}(68 \mathrm{mg}, 0.50 \mathrm{mmol}, 2.0$ equiv.) in the presence of trifluoroacetic acid $(3.8 \mu \mathrm{L}, 0.05 \mathrm{mmol}, 20 \mathrm{~mol}$ $\%)$ in methylene chloride $(125 \mu \mathrm{L})$ was run at $0{ }^{\circ} \mathrm{C}$ for $96 \mathrm{~h}$ to furnish the crude product $\left\{\right.$ exo:endo $=80: 20$, determined by integration of one set of ${ }^{1} \mathrm{H} \mathrm{NMR}$ signals $\left.\left[\delta_{\text {major }}=5.27 \mathrm{ppm}(\mathrm{s}), \delta_{\text {minor }}=5.34 \mathrm{ppm}(\mathrm{d})\right]\right\}$. The crude product was purified by flash chromatography ( silica gel, hexanes/ethyl acetate $=4 / 1$ ) to give pure exo-7I (major diastereomer) as a white solid (45.0 $\mathrm{mg}, 68 \%$ yield) in $99 \%$ ee [determined by HPLC, Chiralpak AS, hexanes:isopropanol $=95: 5,0.75 \mathrm{~mL} / \mathrm{min}, \lambda=220 \mathrm{~nm}, \mathrm{t}$ (major) $=41.8$ $\min , \mathrm{t}($ minor $)=37.6 \mathrm{~min}],[\alpha]_{25}^{\mathrm{D}}=+88.1^{\circ}\left(\mathrm{c} 1.0, \mathrm{CHCl}_{3}\right) ;{ }^{1} \mathrm{H} \mathrm{NMR}\left(400 \mathrm{MHz}, \mathrm{CDCl}_{3}\right)$ $\delta 2.28(\mathrm{~s}, 3 \mathrm{H}), 3.32(\mathrm{~d}, J=6.8 \mathrm{~Hz}, 1 \mathrm{H}), 3.79(\mathrm{dd}, J=2.8,6.4 \mathrm{~Hz}, 2 \mathrm{H}), 5.27(\mathrm{~s}, 1 \mathrm{H}), 6.08$ (bs, 1H), 6.30 (bd, $J=1.2 \mathrm{~Hz} 1 \mathrm{H}), 6.34$ (dd, $J=5.2,7.6 \mathrm{~Hz}, 1 \mathrm{H}), 6.53$ (d, J=7.2 Hz, 1H), $7.33(\mathrm{~s}, 1 \mathrm{H}) ;{ }^{13} \mathrm{C} \mathrm{NMR}\left(100 \mathrm{MHz}, \mathrm{CDCl}_{3}\right) \delta 33.2,42.9,56.1,74.9,76.4,107.3$, 
110.8, 129.5, 138.0, 142.9, 151.6, 173.2, 207.6; IR (neat) v 3420, 2924, 1766, 1713, 1361, 1140; HRMS (ESI/[M+Na] $]^{\dagger}$ ) Calcd. for: $\mathrm{C}_{13} \mathrm{H}_{12} \mathrm{O}_{5} \mathrm{Na}: 271.0582$. Found: 271.0573.

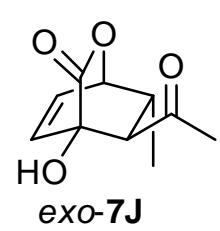

exo-7J 8-acetyl-4-hydroxy-7-methyl-2-oxa-bicyclo[2.2.2]oct-5-en-3-one (7J)exo-7J (major diastereomer)-QD-4 catalyzed ( $4.0 \mathrm{mg}, 0.012 \mathrm{mmol}, 5 \mathrm{~mol}$ \%) reaction of $5 \mathbf{a}(28 \mathrm{mg}, 0.25 \mathrm{mmol})$ and $\mathbf{6 J}(42 \mathrm{mg}, 0.50 \mathrm{mmol}, 2.0$ equiv) in the presence of trifluoroacetic acid $(3.8 \mu \mathrm{L}, 0.05 \mathrm{mmol}, 20 \mathrm{~mol}$ \{exo:endo $=84: 16$, determined by integration of one set of ${ }^{1} \mathrm{H}$ NMR signals $\left[\delta_{\text {major }}=6.37\right.$ ppm (dd), $\delta_{\text {minor }}=6.24 \mathrm{ppm}$ (d)]\}. The crude product was purified by flash chromatography (silica gel, hexanes/ethyl acetate $=4 / 1$ ) to give pure exo-7J (major diastereomer) as a white solid (37.5 mg, 77\% yield) in 99\% ee [determined by HPLC, Chiralcel OD, hexanes:isopropanol $=95: 5,0.25 \mathrm{~mL} / \mathrm{min}, \lambda=215 \mathrm{~nm}, \mathrm{t}$ (major) $=194.5$ $\min , \mathrm{t}($ minor $)=231.8 \mathrm{~min}],[\alpha]_{25}^{\mathrm{D}}=+4.6^{\circ}\left(\mathrm{c} 1.0, \mathrm{CHCl}_{3}\right) ;{ }^{1} \mathrm{H} \mathrm{NMR}\left(400 \mathrm{MHz}, \mathrm{CDCl}_{3}\right)$ $\delta 1.03(\mathrm{~d}, J=6.8 \mathrm{~Hz}, 3 \mathrm{H}), 2.27(\mathrm{~s}, 3 \mathrm{H}), 2.56-2.52(\mathrm{~m}, 1 \mathrm{H}), 2.61(\mathrm{~d}, J=6.0 \mathrm{~Hz}, 1 \mathrm{H}) 3.73$ (s, 1H), $4.93(\mathrm{~s}, 1 \mathrm{H}), 6.37$ (dd, $J=5.2,8.0 \mathrm{~Hz}, 1 \mathrm{H}), 6.47(\mathrm{~d}, J=8.0 \mathrm{~Hz}, 1 \mathrm{H}) ;{ }^{13} \mathrm{C} \mathrm{NMR}$ $\left(100 \mathrm{MHz}, \mathrm{CDCl}_{3}\right) \delta 19.5,33.2,38.7,58.7,76.4,77.4,129.0,138.0,173.5,208.2$; IR (neat) $v 3342,1744,1700,1373,1152 ; \mathrm{HRMS}\left(\mathrm{ESI} /[\mathrm{M}+\mathrm{Na}]^{+}\right.$) Calcd. for: $\mathrm{C}_{10} \mathrm{H}_{12} \mathrm{O}_{4} \mathrm{Na}$ : 219.0657. Found: 219.0637.

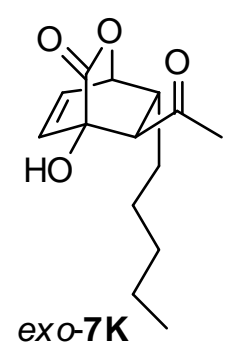

8-acetyl-4-hydroxy-7-pentyl-2-oxa-bicyclo[2.2.2]oct-5-en-3-one (7K)exo-7K (major diasteromer)-QD-4 catalyzed (4.0 mg, $0.012 \mathrm{mmol}, 5 \mathrm{~mol}$ \%) reaction of $5 \mathbf{a}(28 \mathrm{mg}, 0.25 \mathrm{mmol})$ and $\mathbf{6 K}(70 \mathrm{mg}, 0.50 \mathrm{mmol}, 2.0$ equiv) in the presence of trifluoroacetic acid $(3.8 \mu \mathrm{L}, 0.05 \mathrm{mmol}, 20 \mathrm{~mol}$ $\%)$ in methylene chloride $(125 \mu \mathrm{L})$ was run at $-30{ }^{\circ} \mathrm{C}$ for $96 \mathrm{~h}$ to furnish the crude product exo:endo $=75: 25$, determined by integration of one set of ${ }^{1} \mathrm{H}$ NMR signals $\left.\left[\delta_{\text {major }}=6.35 \mathrm{ppm}(\mathrm{dd}), \delta_{\text {minor }}=6.18 \mathrm{ppm}(\mathrm{d})\right]\right\}$. The crude product was purified by flash chromatography (silica gel, hexanes/ethyl acetate $=4 / 1$ ) to give pure exo-7K (major diastereomer) as a white solid ( $44.0 \mathrm{mg}, 70 \%$ yield) in $99 \%$ ee [determined by HPLC, Chiralpak AD, hexanes:isopropanol $=97.5: 2.5,1.0 \mathrm{~mL} / \mathrm{min}, \lambda=215 \mathrm{~nm}, \mathrm{t}$ $($ major $)=52.2 \mathrm{~min}, \mathrm{t}($ minor $)=40.4 \mathrm{~min}],[\alpha]_{25}^{\mathrm{D}}=+14.9^{\circ}\left(\mathrm{c} 1.0, \mathrm{CHCl}_{3}\right) ;{ }^{1} \mathrm{H}$ NMR $(400$ $\left.\mathrm{MHz}, \mathrm{CDCl}_{3}\right) \delta 0.83(\mathrm{t}, J=6.0 \mathrm{~Hz}, 3 \mathrm{H}), 1.32-1.10(\mathrm{~m}, 8 \mathrm{H}), 2.28(\mathrm{~s}, 3 \mathrm{H}), 2.48-2.45(\mathrm{~m}$, $1 \mathrm{H}), 2.67(\mathrm{~d}, J=5.6 \mathrm{~Hz}, 1 \mathrm{H}) 3.72(\mathrm{~s}, 1 \mathrm{H}), 5.02(\mathrm{~s}, 1 \mathrm{H}), 6.35(\mathrm{dd}, J=4.0,7.2 \mathrm{~Hz}, 1 \mathrm{H})$, 
$6.44(\mathrm{~d}, J=7.6 \mathrm{~Hz}, 1 \mathrm{H}) ;{ }^{13} \mathrm{C}$ NMR $\left(100 \mathrm{MHz}, \mathrm{CDCl}_{3}\right) \delta$ 14.1, 22.6, 26.9, 31.6, 33.2, 34.2, 44.4, 57.4, 76.4, 76.8, 129.3, 137.9, 173.8, 208.6; IR $\left(\mathrm{CH}_{2} \mathrm{Cl}_{2}\right)$ v 3345, 2929, 1742 , 1705, 1366, 1155; HRMS (ESI/[M+Na $]^{+}$) Calcd. for: $\mathrm{C}_{14} \mathrm{H}_{20} \mathrm{O}_{4} \mathrm{Na}: 275.1283$. Found: 275.1269 .

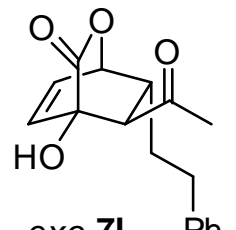

8-acetyl-4-hydroxy-7-phenethyl-2-oxa-bicyclo[2.2.2]oct-5-en-3-one (7L)- exo-7L (major diastereomer)-QD-4 catalyzed (4.0 mg, $0.012 \mathrm{mmol}$, $5 \mathrm{~mol} \%)$ reaction of $\mathbf{5 a}(28 \mathrm{mg}, 0.25 \mathrm{mmol})$ and $\mathbf{6 L}(87 \mathrm{mg}, 0.50 \mathrm{mmol}$, exo-7L Ph 2.0 equiv) in the presence of trifluoroacetic acid $(3.8 \mu \mathrm{L}, 0.05 \mathrm{mmol}, 20$ mol \%) in methylene chloride $(125 \mu \mathrm{L})$ was run at $-20{ }^{\circ} \mathrm{C}$ for $96 \mathrm{~h}$ to furnish the crude product $\left\{\right.$ exo:endo $=94: 6$, determined by integration of one set of ${ }^{1} \mathrm{H}$ NMR signals $\left[\delta_{\text {major }}\right.$ $\left.\left.=6.34 \mathrm{ppm}(\mathrm{dd}), \delta_{\text {minor }}=6.18 \mathrm{ppm}(\mathrm{d})\right]\right\}$. The crude product was purified by flash chromatography (silica gel, hexanes/ethyl acetate $=4 / 1$ ) to give pure exo-7L (major diastereomer) as a white solid (70 mg, $99.0 \%$ yield) in 99\% ee [determined by HPLC, Chiralpak AD, hexanes:isopropanol $=60: 40,0.5 \mathrm{~mL} / \mathrm{min}, \lambda=220 \mathrm{~nm}, \mathrm{t}$ (major) $=19.8$ $\min , \mathrm{t}($ minor $)=13.2 \mathrm{~min}],[\alpha]_{25}^{\mathrm{D}}=+37.8^{\circ}\left(\mathrm{c} 1.0, \mathrm{CHCl}_{3}\right) ;{ }^{1} \mathrm{H} \mathrm{NMR}\left(400 \mathrm{MHz}, \mathrm{CDCl}_{3}\right)$ $\delta 1.55-1.45(\mathrm{~m}, 1 \mathrm{H}), 1.68-1.61(\mathrm{~m}, 1 \mathrm{H}), 2.27(\mathrm{~s}, 3 \mathrm{H}), 2.61-2.56(\mathrm{~m}, 3 \mathrm{H}), 2.73(\mathrm{~d}, J=5.6$ Hz, 1H), $3.76(\mathrm{bs}, 1 \mathrm{H}), 5.04(\mathrm{~s}, 1 \mathrm{H}), 6.34(\mathrm{dd}, J=4.8,6.8 \mathrm{~Hz}, 1 \mathrm{H}), 6.47$ (d, $J=7.2 \mathrm{~Hz}$, $1 \mathrm{H}), 7.10(\mathrm{~d}, J=7.2 \mathrm{~Hz}, 2 \mathrm{H}), 7.33-7.17(\mathrm{~m}, 3 \mathrm{H}) ;{ }^{13} \mathrm{C} \mathrm{NMR}\left(100 \mathrm{MHz}, \mathrm{CDCl}_{3}\right) \delta 33.2$, 33.6, 36.2, 43.9, 57.6, 76.1, 76.7, 126.7, 128.3, 128.9, 129.3, 138.1, 140.5, 173.6, 208.3; IR $\left(\mathrm{CH}_{2} \mathrm{Cl}_{2}\right) \vee 3448,2925,1760,1711,1360,1145 ; \mathrm{HRMS}\left(\mathrm{ESI} /[\mathrm{M}+\mathrm{Na}]^{+}\right)$Calcd. for: $\mathrm{C}_{17} \mathrm{H}_{18} \mathrm{O}_{4} \mathrm{Na}: 309.1103$. Found: 309.1101.

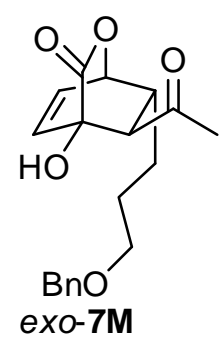

8-acetyl-7-(2-(benzyloxy)ethyl)-4-hydroxy-2-oxa-bicyclo[2.2.2]oct-5en-3-one (7M) exo-7M (major diastereomer) QD-4 catalyzed (2.0 mg, $0.00625 \mathrm{mmol}, 5 \mathrm{~mol} \%)$ reaction of $5 \mathbf{a}(14 \mathrm{mg}, 0.125 \mathrm{mmol})$ and $\mathbf{6 M}$ (55mg, $0.25 \mathrm{mmol}, 2.0$ equiv.) in the presence of TFA $(2.0 \mu \mathrm{L}, 2.85 \mathrm{mg}$, $0.025 \mathrm{mmol}, 20 \mathrm{~mol} \%)$ in methylene chloride $(65 \mu \mathrm{L})$ was run at $-20^{\circ} \mathrm{C}$ for $72 \mathrm{~h}$ to furnish the crude product $\{$ exo:endo $=76: 24$, determined by integration of one set of ${ }^{1} \mathrm{H}$ NMR signals $\left.\left[\delta_{\text {major }}=6.37 \mathrm{ppm}(\mathrm{dd}), \delta_{\text {minor }}=6.21 \mathrm{ppm}(\mathrm{d})\right]\right\}$. The crude product was purified by flash chromatography ( pure exo-7M (major diastereomer) as a colorless solid (29.0 mg, 70\% yield) in $99 \%$ ee [detrmined by HPLC, Chiralcel OD, hexanes:isopropanol $=90: 10,1.0 \mathrm{~mL} / \mathrm{min}, \lambda=220$ 
$\mathrm{nm}, \mathrm{t}($ major $)=50.2 \mathrm{~min}, \mathrm{t}($ minor $)=72.4 \mathrm{~min}] .[\alpha]^{\mathrm{D}}{ }_{25}=15.9^{\circ}\left(c \quad 1.0, \mathrm{CHCl}_{3}\right) ;{ }^{1} \mathrm{H} \mathrm{NMR}$ $\left(400 \mathrm{MHz}, \mathrm{CDCl}_{3}\right) \delta$ 1.22-1.34 (m, 1H), 1.39-1.50 (m, 1H), 1.52-1.66 (m, 2H), $2.30(\mathrm{~s}$, $3 \mathrm{H}), 2.50-2.57(\mathrm{~m}, 1 \mathrm{H}), 2.71(\mathrm{~d}, J=5.6 \mathrm{~Hz}, 1 \mathrm{H}), 3.43(\mathrm{t}, J=6.0 \mathrm{~Hz}, 2 \mathrm{H}), 3.78(\mathrm{~s}, 1 \mathrm{H})$, 4.47 (s, 2H), 5.05 (s, 1H), 6.37 (dd, $J=5.2,8.0 \mathrm{~Hz}, 1 \mathrm{H}), 6.47$ (d, $J=8.0 \mathrm{~Hz}, 1 \mathrm{H}), 7.25-$ $7.38(\mathrm{~m}, 5 \mathrm{H}) ;{ }^{13} \mathrm{C}$ NMR $\left(100 \mathrm{MHz}, \mathrm{CDCl}_{3}\right) \delta 27.48,31.11,33.24,44.09,57.29,69.53$, $73.29,76.25,76.77,127.90,127.93,128.63,129.30,137.92,138.28,173.74,208.50$; IR $\left(\mathrm{CH}_{2} \mathrm{Cl}_{2}\right) \vee 3360,2935,2859,1753,1706,1361,1150$; HRMS (ESI/[M+Na] $\left.{ }^{+}\right)$Calcd. for: $\mathrm{C}_{19} \mathrm{H}_{22} \mathrm{O}_{5} \mathrm{Na}: 353.1365$. Found: 353.1354 .

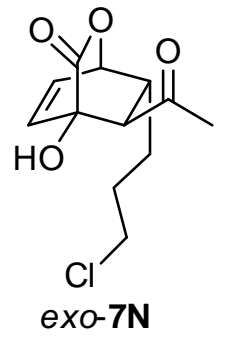

8-acetyl-7-(2-chloroethyl)-4-hydroxy-2-oxa-bicyclo[2.2.2]oct-5-en-3one (7N)- exo-7N-QD-4-catalyzed (2.4 mg, $0.0075 \mathrm{mmol}, 5 \mathrm{~mol} \%)$ reaction of $5 \mathbf{a}(16.8 \mathrm{mg}, 0.15 \mathrm{mmol})$ and $\mathbf{6 N}$ (44mg, $0.3 \mathrm{mmol}, 2.0$ equiv.) in the presence of TFA $(2.3 \mu \mathrm{L}, 3.42 \mathrm{mg}, 0.03 \mathrm{mmol}, 20 \mathrm{~mol} \%)$ in methylene chloride $(75 \mu \mathrm{L})$ was run at $-20^{\circ} \mathrm{C}$ for $120 \mathrm{~h}$ to furnish the crude product exo:endo $=76: 24$, determined by integration of one set of ${ }^{1} \mathrm{H}$ NMR signals $\left.\left[\delta_{\text {major }}=6.42 \mathrm{ppm}(\mathrm{dd}), \delta_{\text {minor }}=6.23 \mathrm{ppm}(\mathrm{d})\right]\right\}$. The crude product was purified by flash chromatography (silica gel, hexanes/ethyl acetate $=5 / 1$ ) to give pure exo-7N (major diastereomer) as a colorless solid (24.5 mg, 63\% yield) in 96\% ee [detrmined by HPLC, Chiralpak $\mathrm{AD}+(R, R)$-Whelk-O1, hexanes:isopropanol $=75: 25,1.0 \mathrm{~mL} / \mathrm{min}, \lambda=215$ $\mathrm{nm}, \mathrm{t}($ major $)=23.6 \mathrm{~min}, \mathrm{t}($ minor $)=21.7 \mathrm{~min}] .[\alpha]_{25}^{\mathrm{D}}=15.9^{\circ}\left(c \quad 1.0, \mathrm{CHCl}_{3}\right) ;{ }^{1} \mathrm{H} \mathrm{NMR}$ $\left(400 \mathrm{MHz}, \mathrm{CDCl}_{3}\right) \delta$ 1.30-1.42 (m, 1H), 1.48-1.59 (m, 1H), 1.72-1.83 (m, 2H), $2.34(\mathrm{~s}$, $3 \mathrm{H}), 2.51-2.60(\mathrm{~m}, 1 \mathrm{H}), 2.74(\mathrm{~d}, J=5.2 \mathrm{~Hz}, 1 \mathrm{H}), 3.52$ (t, $J=6.0 \mathrm{~Hz}, 2 \mathrm{H}), 3.88(\mathrm{~s}, 1 \mathrm{H})$, $5.08(\mathrm{~s}, 1 \mathrm{H}), 6.42(\mathrm{dd}, J=4.8,8.0 \mathrm{~Hz}, 1 \mathrm{H}), 6.52(\mathrm{~d}, J=8.0 \mathrm{~Hz}, 1 \mathrm{H}) ;{ }^{13} \mathrm{C}$ NMR $(100$ $\left.\mathrm{MHz}_{\mathrm{CDCl}}\right) \delta 30.06,31.45,33.31,43.58,44.36,57.34,76.00,76.77,129.15,138.21$, 173.54, 208.33; IR $\left(\mathrm{CH}_{2} \mathrm{Cl}_{2}\right)$ v 3718, 3342, 2943, 1748, 1703, 1427, 1368, 1156; HRMS $\left(\mathrm{ESI} /[\mathrm{M}+\mathrm{Na}]^{+}\right)$Calcd. for: $\mathrm{C}_{12} \mathrm{H}_{15} \mathrm{ClO}_{4} \mathrm{Na}: 281.0557$. Found: 281.0548 .

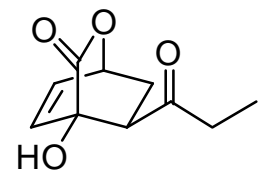

exo-70

4-hydroxy-8-propionyl-2-oxa-bicyclo[2.2.2]oct-5-en-3-one (70)- ехо70 (major diastereomer)-QD-4 catalyzed (4.0 mg, $0.012 \mathrm{mmol}, 5 \mathrm{~mol}$ \%) reaction of $5 \mathbf{a}(28 \mathrm{mg}, 0.25 \mathrm{mmol})$ and $\mathbf{6 0}(42 \mathrm{mg}, 0.50 \mathrm{mmol}, 2.0$ equiv) in the presence of trifluoroacetic acid $(3.8 \mu \mathrm{L}, 0.05 \mathrm{mmol}, 20 \mathrm{~mol}$ $\%)$ in methylene chloride $(125 \mu \mathrm{L})$ was run at $-20{ }^{\circ} \mathrm{C}$ for $72 \mathrm{~h}$ to furnish the crude product $\left\{\right.$ exo:endo $=97: 3$, determined by integration of one set of ${ }^{1} \mathrm{H}$ NMR signals $\left[\delta_{\text {major }}=3.11\right.$ 
ppm (dd), $\left.\left.\delta_{\text {minor }}=2.94 \mathrm{ppm}(\mathrm{dd})\right]\right\}$. The crude product was purified by flash chromatography (silica gel, hexanes/ethyl acetate $=4 / 1$ ) to give pure exo-70 (major diastereomer) as a white solid (47.20 mg, $96.0 \%$ yield) in 99\% ee [determined by HPLC, Chiralpak AD, hexanes:isopropanol $=85: 15,1.0 \mathrm{~mL} / \mathrm{min}, \lambda=220 \mathrm{~nm}, \mathrm{t}$ (major) $=18.6$ min, $\mathrm{t}($ minor $)=16.8 \mathrm{~min}],[\alpha]^{\mathrm{D}}{ }_{25}=-71.4^{\circ}\left(\mathrm{c} 1.0, \mathrm{CHCl}_{3}\right) ;{ }^{1} \mathrm{H} \mathrm{NMR}\left(400 \mathrm{MHz}, \mathrm{CDCl}_{3}\right) \delta$ $1.02(\mathrm{t}, J=7.2 \mathrm{~Hz}, 3 \mathrm{H}), 1.97(\mathrm{t}, J=12.0 \mathrm{~Hz}, 1 \mathrm{H}), 2.25-2.19(\mathrm{~m}, 1 \mathrm{H}), 2.54-2.46(\mathrm{~m}, 1 \mathrm{H})$, 2.67-2.59 (m, 1H), $3.11(\mathrm{dd}, J=6.0,10.8 \mathrm{~Hz}, 1 \mathrm{H}), 3.75$ (bs, 1H), 5.23 (bs, 1H), 6.46-6.38 (m, 2H); ${ }^{13} \mathrm{C}$ NMR (100 MHz, $\left.\mathrm{CDCl}_{3}\right) \delta$ 7.4, 31.4, 38.9, 48.9, 73.1, 76.3, 130.5, 138.3, 174.0, 211.2; IR (neat) $v$ 3332, 2951, 1746, 1712, 1402, 1360; HRMS (CI/[M+H $\left.]^{+}\right)$ Calcd. for: $\mathrm{C}_{10} \mathrm{H}_{13} \mathrm{O}_{4}$ : 197.0814. Found: 197.0814.

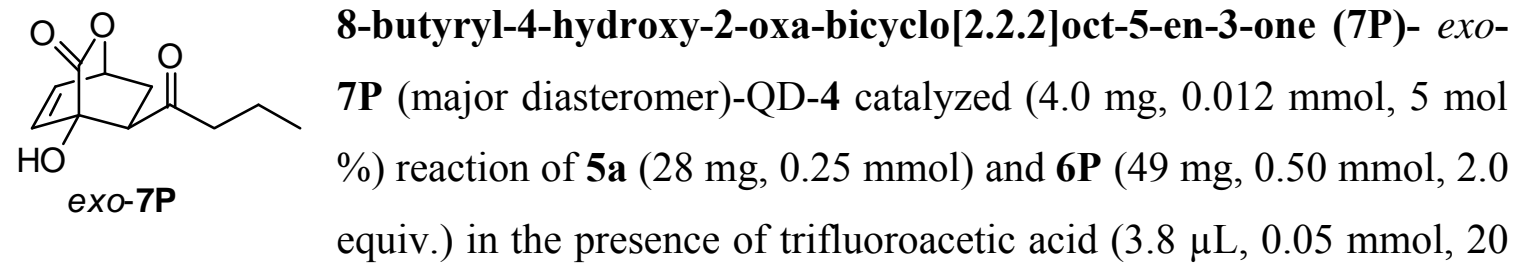

mol \%) in methylene chloride $(125 \mu \mathrm{L})$ was run at $-20^{\circ} \mathrm{C}$ for $72 \mathrm{~h}$ to furnish the crude product $\left\{\right.$ exo:endo $=92: 8$, determined by integration of one set of ${ }^{1} \mathrm{H}$ NMR signals $\left[\delta_{\text {major }}\right.$ $\left.\left.=3.10 \mathrm{ppm}(\mathrm{dd}), \delta_{\text {minor }}=2.93 \mathrm{ppm}(\mathrm{dd})\right]\right\}$. The crude product was purified by flash chromatography (silica gel, hexanes/ethyl acetate $=4 / 1$ ) to give pure exo-7P (major diastereomer) as a pale yellow liquid ( $43.2 \mathrm{mg}, 82 \%$ yield) in $99 \%$ ee [determined by HPLC, Chiralpak AD, hexanes:isopropanol $=90: 10,1.0 \mathrm{~mL} / \mathrm{min}, \lambda=220 \mathrm{~nm}, \mathrm{t}$ (major) $=28.2 \mathrm{~min}, \mathrm{t}($ minor $)=24.3 \mathrm{~min}],[\alpha]_{25}^{\mathrm{D}}=-62.8^{\circ}\left(\mathrm{c} 1.14, \mathrm{CHCl}_{3}\right) ;{ }^{1} \mathrm{H} \mathrm{NMR}(400 \mathrm{MHz}$, $\left.\mathrm{CDCl}_{3}\right) \delta 0.87(\mathrm{t}, J=7.6 \mathrm{~Hz}, 3 \mathrm{H}), 1.57(\mathrm{sext}, J=7.2 \mathrm{~Hz}, 2 \mathrm{H}), 1.97(\mathrm{t}, J=11.6 \mathrm{~Hz}, 1 \mathrm{H})$, 2.21 ( td, $J=4.8,13.6 \mathrm{~Hz}, 1 \mathrm{H}), 2.45(\mathrm{td}, J=7.6,17.6 \mathrm{~Hz}, 1 \mathrm{H}), 2.58(\mathrm{td}, J=7.2,17.6 \mathrm{~Hz}$, $1 \mathrm{H}), 3.10$ (dd, $J=6.0,11.2 \mathrm{~Hz}, 1 \mathrm{H}), 3.76$ ( bs, $1 \mathrm{H}), 5.22$ (d, $J=1.2 \mathrm{~Hz}, 1 \mathrm{H}), 6.45-6.38$ (m, 2H); ${ }^{13} \mathrm{C}$ NMR $\left(100 \mathrm{MHz}, \mathrm{CDCl}_{3}\right) \delta 13.8,16.7,31.3,47.4,49.0,73.1,76.3,130.5$, 138.3, 174.0, 210.7; IR (neat) v 3447, 2962, 2933, 2876, 1756, 1711, 1363, 1142; HRMS $\left(\mathrm{CI} /[\mathrm{M}+\mathrm{H}]^{+}\right)$Calcd. for: $\mathrm{C}_{11} \mathrm{H}_{15} \mathrm{O}_{4}: 211.09704$. Found: 211.09703 .

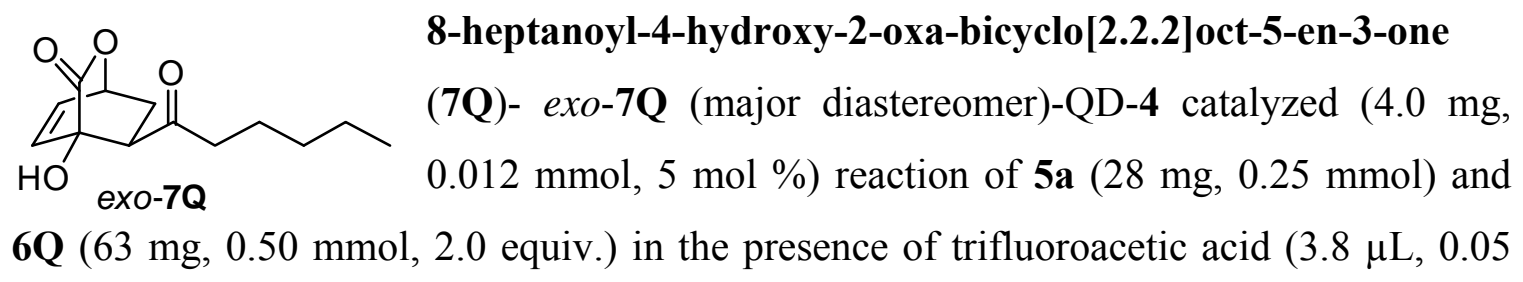


mmol, $20 \mathrm{~mol} \%)$ in methylene chloride $(125 \mu \mathrm{L})$ was run at $-20^{\circ} \mathrm{C}$ for $72 \mathrm{~h}$ to furnish the crude product $\left\{\right.$ exo:endo $=93: 7$, determined by integration of one set of ${ }^{1} \mathrm{H}$ NMR signals $\left[\delta_{\text {major }}=3.13 \mathrm{ppm}(\mathrm{dd}), \delta_{\text {minor }}=2.95 \mathrm{ppm}(\mathrm{dd})\right]$. The crude product was purified by flash chromatography ( silica gel, hexanes/ethyl acetate $=4 / 1$ ) to give pure exo-7Q (major diastereomer) as a white solid (54.10 mg, $91.0 \%$ yield) in $96 \%$ ee [determined by HPLC, Chiralpak AD, hexanes:isopropanol $=90: 10,1.0 \mathrm{~mL} / \mathrm{min}, \lambda=220 \mathrm{~nm}, \mathrm{t}$ (major) $=$ $22.4 \mathrm{~min}, \mathrm{t}($ minor $)=19.8 \mathrm{~min}],[\alpha]^{\mathrm{D}}{ }_{25}=-62.4^{\circ}\left(c\right.$ 1.25, $\left.\mathrm{CHCl}_{3}\right) ;{ }^{1} \mathrm{H} \mathrm{NMR}(400 \mathrm{MHz}$, $\left.\mathrm{CDCl}_{3}\right) \delta 0.87(\mathrm{t}, J=6.8 \mathrm{~Hz}, 3 \mathrm{H}), 1.32-1.21(\mathrm{~m}, 4 \mathrm{H}), 1.56$ (quin, $\left.J=7.6 \mathrm{~Hz}, 1 \mathrm{H}\right), 1.99$ (t, $J=12.0 \mathrm{~Hz}, 1 \mathrm{H}), 2.24(\mathrm{td}, J=4.4,13.2 \mathrm{~Hz}, 1 \mathrm{H}), 2.48(\mathrm{td}, J=7.2,17.6 \mathrm{~Hz}, 1 \mathrm{H}), 2.62(\mathrm{td}$, $J=8.0,17.6 \mathrm{~Hz}, 1 \mathrm{H}), 3.12(\mathrm{dd}, J=5.2,10.8 \mathrm{~Hz}, 1 \mathrm{H}), 3.75(\mathrm{bs}, 1 \mathrm{H}), 5.25(\mathrm{~d}, J=1.2 \mathrm{~Hz}$, 1H), 6.49-6.41 (m, 2H); ${ }^{13} \mathrm{C}$ NMR (100 MHz, $\left.\mathrm{CDCl}_{3}\right) \delta 14.1,22.6,22.9,31.3,31.4,45.5$, 49.0, 73.0, 76.3, 130.4, 138.3, 174.0, 210.8; IR (neat) v 3334, 2952, 2932, 2871, 1736, 1712, 1422, 1366, 1158; HRMS (EI/[M+H] $]^{+}$Calcd. for: $\mathrm{C}_{13} \mathrm{H}_{19} \mathrm{O}_{4}$ : 239.1283. Found: 238.1282 .

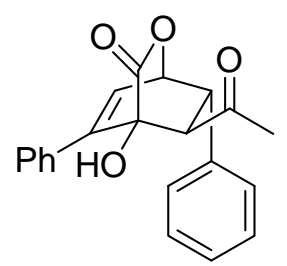

exo-7A'

\section{8-acetyl-4-hydroxy-5,7-diphenyl-2-oxa-bicyclo[2.2.2]oct-5-en-3-one}

(7A') exo-7A' (major diastereomer)-QD-4 catalyzed (2.5 mg, 0.007 mmol, $5 \mathrm{~mol} \%)$ reaction of $5 \mathbf{b}(28.3 \mathrm{mg}, 0.15 \mathrm{mmol})$ and $\mathbf{6 A}(43 \mathrm{mg}$, $0.50 \mathrm{mmol}, 2.0$ equiv) in the presence of trifluoroacetic acid $(2.5 \mu \mathrm{L}$, $0.025 \mathrm{mmol}, 20 \mathrm{~mol} \%)$ in methylene chloride $(75 \mu \mathrm{L})$ was run at $0{ }^{\circ} \mathrm{C}$ for $96 \mathrm{~h}$ to furnish the crude product $\{$ exo:endo $=83: 17$, determined by integration of one set of ${ }^{1} \mathrm{H}$ NMR signals $\left.\left[\delta_{\text {major }}=5.15 \mathrm{ppm}(\mathrm{dd}), \delta_{\text {minor }}=5.29 \mathrm{ppm}(\mathrm{s})\right]\right\}$. The crude product was purified by flash chromatography (silica gel, hexanes/ethyl acetate $=4 / 1$ ) to give pure exo-7A' (major diastereomer) as a pale yellow solid (34.0 mg, 67\% yield) in 96\% ee [determined by HPLC, Chiralpak AD, hexanes:isopropanol $=90: 10,1.0$ $\mathrm{mL} / \mathrm{min}, \lambda=220 \mathrm{~nm}, \mathrm{t}$ (major) $=27.5 \mathrm{~min}, \mathrm{t}$ (minor) $=17.9 \mathrm{~min}],[\alpha]^{\mathrm{D}}{ }_{25}=+96.6^{\circ}(c 1.0$, $\left.\mathrm{CHCl}_{3}\right) ;{ }^{1} \mathrm{H} \mathrm{NMR}\left(400 \mathrm{MHz}, \mathrm{CDCl}_{3}\right) \delta 2.23(\mathrm{~s}, 3 \mathrm{H}), 3.60(\mathrm{~d}, J=6.8 \mathrm{~Hz}, 1 \mathrm{H}), 3.85$ (dd, $J$ $=2.8,6.8 \mathrm{~Hz}, 1 \mathrm{H}), 3.88(\mathrm{bs}, 1 \mathrm{H}), 5.15(\mathrm{dd}, J=3.6,5.6 \mathrm{~Hz}, 1 \mathrm{H}), 6.38(\mathrm{~d}, J=4.8 \mathrm{~Hz}, 1 \mathrm{H})$, 7.53-7.17(m, 10H); ${ }^{13} \mathrm{C}$ NMR $\left(100 \mathrm{MHz}, \mathrm{CDCl}_{3}\right) \delta 33.7,50.3,59.3,76.6,77.8,125.0$, $128.1,128.4,128.6,128.7,129.3,129.4,133.2,138.3,148.2,173.6,208.0 ; \operatorname{IR}\left(\mathrm{CH}_{2} \mathrm{Cl}_{2}\right) v$ 3446, 2923, 1763, 1710, 1140; HRMS (ESI/[M+Na $]^{+}$) Calcd. for: $\mathrm{C}_{21} \mathrm{H}_{18} \mathrm{O}_{4} \mathrm{Na}$ : 357.1127. Found: 357.1102 . 


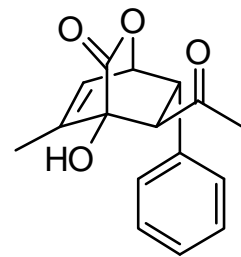

exo-7A"

\section{8-acetyl-4-hydroxy-5-methyl-7-phenyl-2-oxa-bicyclo[2.2.2]oct-5-en-3-}

one (7A") -ехо-7A"- (major diastereomer)-QD-4 catalyzed (2.5 mg, $0.007 \mathrm{mmol}, 5 \mathrm{~mol} \%)$ reaction of $5 \mathbf{c}(18.5 \mathrm{mg}, 0.15 \mathrm{mmol})$ and $\mathbf{6 A}(43$ $\mathrm{mg}, 0.50 \mathrm{mmol}, 2.0$ equiv) in the presence of trifluoroacetic acid $(2.5 \mu \mathrm{L}$, $0.025 \mathrm{mmol}, 20 \mathrm{~mol} \%)$ in methylene chloride $(75 \mu \mathrm{L})$ was run at $0{ }^{\circ} \mathrm{C}$ for $96 \mathrm{~h}$ to furnish the crude product $\{$ exo:endo $=80: 20$, determined by integration of one set of ${ }^{1} \mathrm{H}$ NMR signals $\left.\left[\delta_{\text {major }}=4.97 \mathrm{ppm}(\mathrm{s}), \delta_{\text {minor }}=5.13 \mathrm{ppm}(\mathrm{s})\right]\right\}$. The crude product was purified by flash chromatography ( exo-7A" (major diastereomer) as a semi solid (19.0 $\mathrm{mg}, 47 \%$ yield) in $99 \%$ ee [determined by HPLC, Chiralpak AD, hexanes:isopropanol = 95:5, $1.0 \mathrm{~mL} / \mathrm{min}, \lambda=220$ $\mathrm{nm}, \mathrm{t}($ major $)=28.8 \mathrm{~min}, \mathrm{t}($ minor $)=24.9 \mathrm{~min}],[\alpha]^{\mathrm{D}}{ }_{25}=+71.7^{\circ}\left(\mathrm{c} 0.70, \mathrm{CHCl}_{3}\right) ;{ }^{1} \mathrm{H}$ NMR (400 MHz, $\left.\mathrm{CDCl}_{3}\right) \delta$ 2.01(s, 3H), $2.16(\mathrm{~s}, 3 \mathrm{H}), 3.28(\mathrm{~d}, J=4.4 \mathrm{~Hz}, 1 \mathrm{H}), 3.68$ (s, $1 \mathrm{H}), 3.76(\mathrm{~s}, 1 \mathrm{H}), 4.97(\mathrm{~s}, 1 \mathrm{H}), 6.06(\mathrm{~s}, 1 \mathrm{H}), 7.13-7.12(\mathrm{~m}, 2 \mathrm{H}), 7.30-7.23(\mathrm{~m}, 3 \mathrm{H}) ;{ }^{13} \mathrm{C}$ NMR $\left(100 \mathrm{MHz} \mathrm{CDCl}_{3}\right) \delta 14.5,33.3,50.4,58.4,76.6,77.9,122.8,128.0,128.3,129.3$, 138.5, 146.5, 173.9, 208.2; IR $\left(\mathrm{CH}_{2} \mathrm{Cl}_{2}\right) \vee 3417,2924,1723,1709,1660,1413,1359$, 1294; HRMS (ESI/[M+Na] $]^{+}$) Calcd. for: $\mathrm{C}_{16} \mathrm{H}_{16} \mathrm{O}_{4} \mathrm{Na}: 295.0946$. Found: 295.0948 .

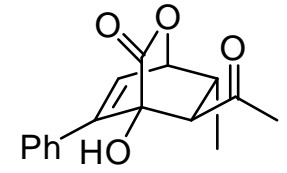

exo-7 $\mathbf{J}^{\prime}$

8-acetyl-4-hydroxy-7-methyl-5-phenyl-2-oxa-bicyclo[2.2.2]oct-5-en3-one (7J')-exo-7J' (major diastereomer)-QD-4 catalyzed (2.5 mg, $0.007 \mathrm{mmol}, 5 \mathrm{~mol} \%)$ reaction of $\mathbf{5 b}(28.3 \mathrm{mg}, 0.15 \mathrm{mmol})$ and $\mathbf{6 J}(26$ $\mathrm{mg}, 0.30 \mathrm{mmol}, 2.0$ equiv) in the presence of trifluoroacetic acid (2.5 $\mu \mathrm{L}, 0.025 \mathrm{mmol}, 20 \mathrm{~mol} \%)$ in methylene chloride $(75 \mu \mathrm{L})$ was run at $0{ }^{\circ} \mathrm{C}$ for $96 \mathrm{~h}$ to furnish the crude product $\left\{\right.$ exo:endo $=80: 20$, determined by integration of one set of ${ }^{1} \mathrm{H}$ NMR signals $\left.\left[\delta_{\text {major }}=6.40 \mathrm{ppm}(\mathrm{t}), \delta_{\text {minor }}=6.49 \mathrm{ppm}(\mathrm{dd})\right]\right\}$. The crude product was purified by flash chromatography ( exo-7J' (major diastereomer) as a white solid (25.7 $\mathrm{mg}, 63 \%$ yield) in $96 \%$ ee [determined by HPLC, Chiralpak AD, hexanes:isopropanol =90:10, $1.0 \mathrm{~mL} / \mathrm{min}, \lambda=220$ $\mathrm{nm}, \mathrm{t}($ major $)=29.7 \mathrm{~min}, \mathrm{t}($ minor $)=22.9 \mathrm{~min}],[\alpha]^{\mathrm{D}}{ }_{25}=-40.6^{\circ}\left(\mathrm{c} 1.05, \mathrm{CHCl}_{3}\right) ;{ }^{1} \mathrm{H}$ NMR (400 MHz, $\left.\mathrm{CDCl}_{3}\right) \delta$ 1.11-1.08 (m, 3H), $2.33(\mathrm{~d}, J=5.6 \mathrm{~Hz}, 3 \mathrm{H}), 2.68-2.67$ (m, $1 \mathrm{H}), 2.85$ (t, $J=4.8 \mathrm{~Hz}, 1 \mathrm{H}), 3.77$ (d, $J=4.4 \mathrm{~Hz}, 1 \mathrm{H}), 5.02(\mathrm{dd}, J=4.8,8.8 \mathrm{~Hz}, 1 \mathrm{H})$, $6.41(\mathrm{~d}, J=4.8 \mathrm{~Hz}, 1 \mathrm{H}), 7.44-7.34(\mathrm{~m}, 5 \mathrm{H}) ;{ }^{13} \mathrm{C} \mathrm{NMR}\left(100 \mathrm{MHz}, \mathrm{CDCl}_{3}\right) \delta 19.7,33.6$, $39.3,59.4,77.0,124.7,128.5,128.6,129.1,133.2,173.7,208.4 ; \mathrm{IR}\left(\mathrm{CH}_{2} \mathrm{Cl}_{2}\right) \vee 3321$, 
2977, 1746, 1709, 1428, 1269, 1146; HRMS (ESI/[M+Na] $]^{+}$) Calcd. for: $\mathrm{C}_{16} \mathrm{H}_{16} \mathrm{O}_{4} \mathrm{Na}$ : 295.0946. Found: 295.0933.

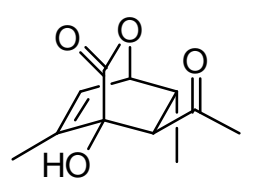

exo-7J"

8-acetyl-4-hydroxy-5,7-dimethyl-2-oxa-bicyclo[2.2.2]oct-5-en-3-one (7J") - exo-7J" (major diastereomer)-QD-4 catalyzed (4.0 mg, 0.012 mmol, $5 \mathrm{~mol} \%)$ reaction of $\mathbf{5 c}(31.5 \mathrm{mg}, 0.25 \mathrm{mmol})$ and $\mathbf{6 J}(42 \mathrm{mg}$, $0.50 \mathrm{mmol}, 2.0$ equiv) in the presence of trifluoroacetic acid $(3.8 \mu \mathrm{L}$, $0.05 \mathrm{mmol}, 20 \mathrm{~mol} \%)$ in methylene chloride $(125 \mu \mathrm{L})$ was run at $0{ }^{\circ} \mathrm{C}$ for $96 \mathrm{~h}$ to furnish the crude product exo:endo $=73: 27$, determined by integration of one set of ${ }^{1} \mathrm{H}$ NMR signals $\left.\left[\delta_{\text {major }}=6.05 \mathrm{ppm}(\mathrm{dd}), \delta_{\text {minor }}=6.13 \mathrm{ppm}(\mathrm{dd})\right]\right\}$. The crude product was purified by flash chromatography (silica gel, hexanes/ethyl acetate $=4 / 1$ ) to give pure exo-7J" (major diastereomer) as a colorless solid (30.0 mg, 57\% yield) in 98\% ee [determined by HPLC, Chiralpak AD, hexanes:isopropanol $=95: 5,1.0 \mathrm{~mL} / \mathrm{min}, \lambda=215 \mathrm{~nm}, \mathrm{t}$ (major) $=$ $17.7 \mathrm{~min}, \mathrm{t}$ (minor) $=16.4 \mathrm{~min}],[\alpha]^{\mathrm{D}}{ }_{25}=-19.8^{\circ}\left(\mathrm{c} 0.85, \mathrm{CHCl}_{3}\right) ;{ }^{1} \mathrm{H}$ NMR $(400 \mathrm{MHz}$, $\left.\mathrm{CDCl}_{3}\right) \delta 1.01(\mathrm{dd}, J=2.8,6.8 \mathrm{~Hz}, 3 \mathrm{H}), 1.90(\mathrm{dd}, J=2.0,3.2 \mathrm{~Hz}, 3 \mathrm{H}), 2.26(\mathrm{~d}, J=3.2$ $\mathrm{Hz}, 1 \mathrm{H}), 2.53-2.50(\mathrm{~m}, 2 \mathrm{H}) 3.68$ (bs, $1 \mathrm{H}), 4.83$ (dd, $J=1.2,2.4 \mathrm{~Hz}, 1 \mathrm{H}), 6.05$ (dd, $J=$ 2.0, $3.2 \mathrm{~Hz}, 1 \mathrm{H}) ;{ }^{13} \mathrm{C} \mathrm{NMR}\left(100 \mathrm{MHz}, \mathrm{CDCl}_{3}\right) \delta 14.35,19.7,33.2,39.4,58.4,77.1,77.7$, 122.3, 146.5, 174.1, 208.6; IR $\left(\mathrm{CH}_{2} \mathrm{Cl}_{2}\right)$ v 3373, 2928, 1746, 1709, 1653, 1366, 1149; HRMS (ESI/[M+Na] $]^{+}$) Calcd. for: $\mathrm{C}_{11} \mathrm{H}_{14} \mathrm{O}_{4} \mathrm{Na}: 233.0790$. Found: 233.0780 .

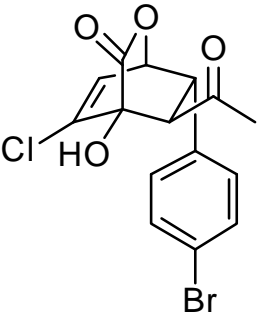

exo-7D'

8-acetyl-5-chloro-7-(4-bromophenyl)-4-hydroxy-2-oxa-bicyclo [2.2.2] $]$ ct-5-en-3-one (7D')- exo-7D' - (major diastereomer)-QD-4 catalyzed (4.0 mg, $0.012 \mathrm{mmol}, 5 \mathrm{~mol} \%)$ reaction of $5 \mathbf{d}(36.5 \mathrm{mg}, 0.25$ $\mathrm{mmol}$ ) and $6 \mathrm{D}$ (112 $\mathrm{mg}, 0.50 \mathrm{mmol}, 2.0$ equiv) in the presence of trifluoroacetic acid $(3.8 \mu \mathrm{L}, 0.05 \mathrm{mmol}, 20 \mathrm{~mol} \%)$ in methylene chloride $(125 \mu \mathrm{L})$ was run at $0{ }^{\circ} \mathrm{C}$ for $96 \mathrm{~h}$ to furnish the crude product $\left\{\right.$ exo:endo $=67: 33$, determined by integration of one set of ${ }^{1} \mathrm{H}$ NMR signals $\left[\delta_{\text {major }}=5.05\right.$ ppm (d), $\left.\left.\delta_{\text {minor }}=5.18 \mathrm{ppm}(\mathrm{d})\right]\right\}$. The crude product was purified by flash chromatography (silica gel, hexanes/ethyl acetate $=4 / 1$ ) to give pure exo-7D' (major diastereomer) as a white solid (38.5 $\mathrm{mg}, 42 \%$ yield) in $90 \%$ ee [determined by HPLC, Chiralcel OD, hexanes:isopropanol $=90: 10,1.0 \mathrm{~mL} / \mathrm{min}, \lambda=220 \mathrm{~nm}, \mathrm{t}$ (major) $=31.7$ min, $\mathrm{t}($ minor $)=43.1 \mathrm{~min}],[\alpha]^{\mathrm{D}}{ }_{25}=+98.4^{\circ}\left(\mathrm{c} 1.0, \mathrm{CHCl}_{3}\right) ;{ }^{1} \mathrm{H} \mathrm{NMR}\left(400 \mathrm{MHz}, \mathrm{CDCl}_{3}\right)$ $\delta 2.22(\mathrm{~s}, 3 \mathrm{H}), 3.41(\mathrm{~d}, J=6.4 \mathrm{~Hz}, 1 \mathrm{H}), 3.76-3.74(\mathrm{~m}, 1 \mathrm{H}), 3.93(\mathrm{bs}, 1 \mathrm{H}), 5.05(\mathrm{~d}, J=3.6$ 
$\mathrm{Hz}, 1 \mathrm{H}), 6.38(\mathrm{~d}, J=5.6 \mathrm{~Hz}, 1 \mathrm{H}), 7.02(\mathrm{~d}, J=7.2 \mathrm{~Hz}, 2 \mathrm{H}), 7.47(\mathrm{~d}, J=7.6 \mathrm{~Hz}, 2 \mathrm{H}) ;{ }^{13} \mathrm{C}$ NMR $\left(100 \mathrm{MHz}, \mathrm{CDCl}_{3}\right) \delta 33.6,49.7,57.8,76.1,122.8,124.8,129.7,132.6,136.5$, 139.7, 140.2, 171.4, 206.7; IR $\left(\mathrm{CH}_{2} \mathrm{Cl}_{2}\right) \vee 3424,2924,1768,1717,1362,1145$; HRMS (ESI/[M+Na] ${ }^{+}$) Calcd. for: $\mathrm{C}_{15} \mathrm{H}_{12} \mathrm{BrClO}_{4} \mathrm{Na}: 392.9505$. Found: 392.9486 .

\section{Reaction Condition and Substrate Scope}

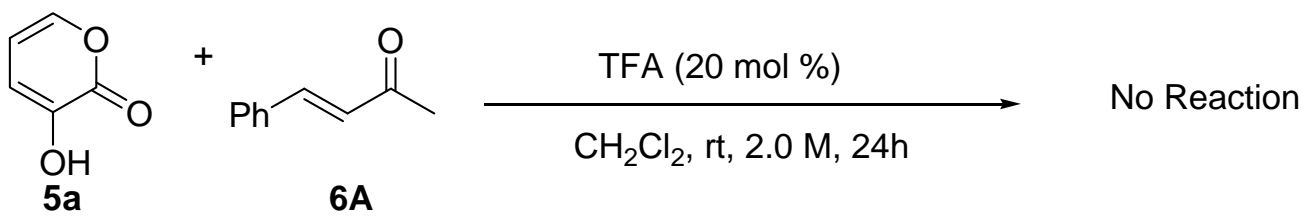

At room temperature, to a solution of 2-pyrone $5 \mathbf{a}(0.25 \mathrm{mmol})$, trans-4-phenyl-3-buten2-one 6A ( $0.50 \mathrm{mmol}, 2$ equiv.) in methylene chloride $(125 \mu \mathrm{L})$ and trifluroacetic acid (3.8 $\mu \mathrm{L}, 0.05 \mathrm{mmol}, 20 \mathrm{~mol} \%$ ) were added sequentially. The resulting reaction mixture was kept at room temperature for $24 \mathrm{~h}$. No reaction product was detected. Only starting materials were recovered.

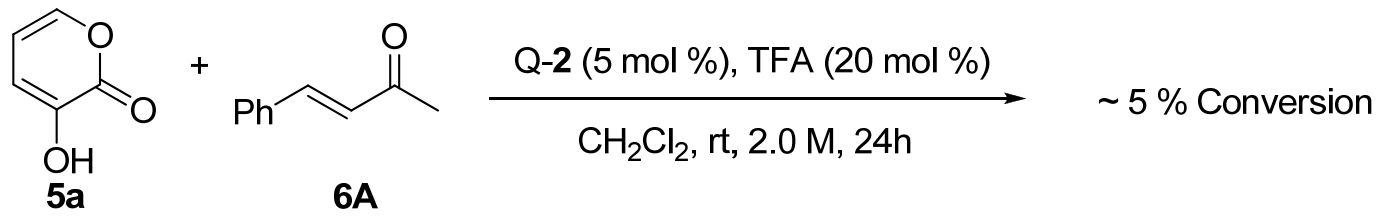

At room temperature, to a solution of 2-pyrone 5a $(0.15 \mathrm{mmol})$, catalyst Q-2 $(0.0075$ mmol, $5 \mathrm{~mol} \%$ ) in methylene chloride ( $75 \mu \mathrm{L})$, trifluroacetic acid $(2.3 \mu \mathrm{L}, 0.03 \mathrm{mmol}, 20$ mol \%) and dienophiles 6A ( $0.3 \mathrm{mmol}, 2$ equiv.) were added. The resulting reaction mixture was kept at room temperature for $24 \mathrm{~h}$. Approximately $5 \%$ of reaction product was detected, rest of starting materials were recovered.

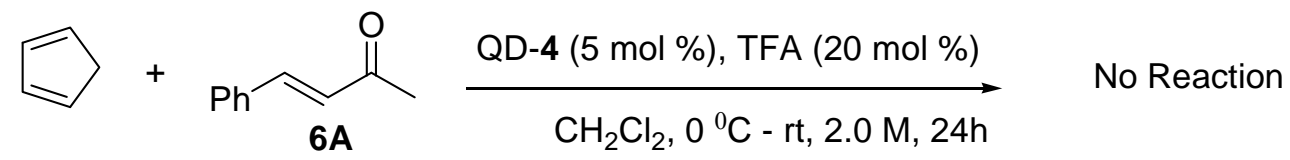

At $0{ }^{0} \mathrm{C}$, to a solution of trans-4-phenyl-3-buten-2-one 6A $(0.25 \mathrm{mmol})$, catalyst QD-4 $(0.025 \mathrm{mmol}, 10 \mathrm{~mol} \%)$ in methylene chloride $(125 \mu \mathrm{L})$ trifluroacetic acid $(7.6 \mu \mathrm{L}, 40$ mol\%) and cyclopentadiene $(1.50 \mathrm{mmol}, 123 \mu \mathrm{L})$ were added sequentially. The resulting reaction mixture was warmed to room temperature and then kept at that temperature for $24 \mathrm{~h}$. No reaction product was detected. Only pure trans-4-phenyl-3-buten-2-one was recovered. 


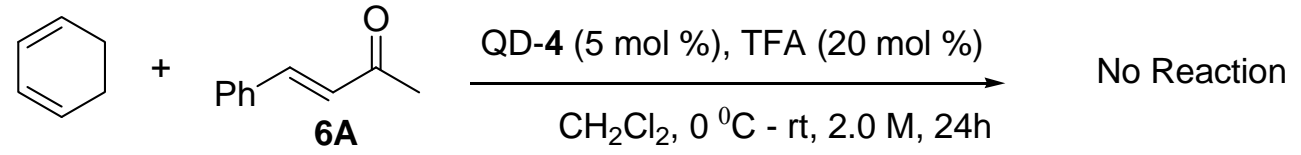

At room temperature, to a solution of trans-4-phenyl-3-buten-2-one $\mathbf{6 A}(0.25 \mathrm{mmol})$, catalyst QD-4 $(0.025 \mathrm{mmol}, 10 \mathrm{~mol} \%)$ in methylene chloride $(125 \mu \mathrm{L})$ trifluroacetic acid $(7.6 \mu \mathrm{L}, 40 \mathrm{~mol} \%)$ and 1,3-cyclohexadiene $(0.50 \mathrm{mmol}, 48 \mu \mathrm{L})$ were added sequentially. The resulting reaction mixture was kept at room temperature for $24 \mathrm{~h}$. No reaction product was detected. Only starting materials were recovered.

\section{Synthetic Transformation of 7A to Chiral Dihydrophenols via Decarboxylation:}

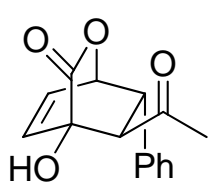

$7 A$
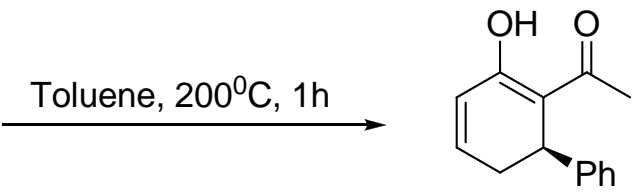

10

1-(2-hydroxy-6-phenylcyclohexa-1,3-dienyl)ethanone (10)- A solution of exo-adduct 7A $(162 \mathrm{mg}, 0.62 \mathrm{mmol})$ in dry toluene $(7.0 \mathrm{~mL})$ was stirred at $200^{\circ} \mathrm{C}$ in a sealed tube for one hour. The solvent was removed under vacuum. The residue was subjected to flash chromatography ( pale yellow solid (123 mg, 93\% yield, mp $65^{\circ} \mathrm{C}$ ) in $95 \%$ ee [determined by HPLC, Chiralcel OD, hexanes:isopropanol $=95: 5,1.0 \mathrm{~mL} / \mathrm{min}, \lambda=220 \mathrm{~nm}, \mathrm{t}$ (major) $=7.8 \mathrm{~min}$, $\mathrm{t}($ minor $)=6.1 \mathrm{~min}],[\alpha]^{\mathrm{D}}{ }_{25}=+267.1^{\circ}\left(\mathrm{c} 1, \mathrm{CHCl}_{3}\right) ;{ }^{1} \mathrm{H} \mathrm{NMR}\left(400 \mathrm{MHz}, \mathrm{CDCl}_{3}\right) \delta 1.89$ (s, 3H), $3.18-2.98(\mathrm{~m}, 2 \mathrm{H}), 4.33$ (q, $J=4 \mathrm{~Hz}, 1 \mathrm{H}), 5.55$ ( bd, $J=9.6 \mathrm{~Hz}, 1 \mathrm{H}), 5.79-5.76$ $(\mathrm{m}, 1 \mathrm{H}), 7.23-7.15(\mathrm{~m}, 3 \mathrm{H}), 7.28(\mathrm{t}, J=7.2 \mathrm{~Hz}, 2 \mathrm{H}) ;{ }^{13} \mathrm{C} \mathrm{NMR}\left(100 \mathrm{MHz}, \mathrm{CDCl}_{3}\right) \delta$ 26.3, 31.5, 43.9, 108.9, 119.0, 126.9, 127.6, 129.2, 130.5, 144.8, 179.2, 201.1; IR (neat) $v$ 3022, 1580, 1362, 1247, 1183, 1075; HRMS (ESI/[M+Na] $]^{+}$Calcd. for: $\mathrm{C}_{14} \mathrm{H}_{14} \mathrm{O}_{2} \mathrm{Na}$ : 237.122. Found: 237.125. 


\section{Relative and Absolute Cofiguration Assignment of Cycloadducts 7}

5.1 Assignment of the absolute configuration of exo-7D and 7D' by X-ray analysis.

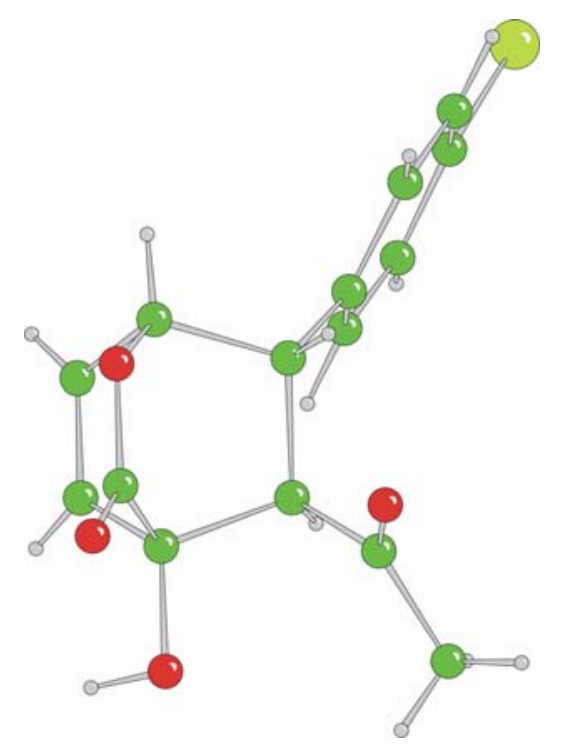

S-Figure 3. X-ray structure of 7D

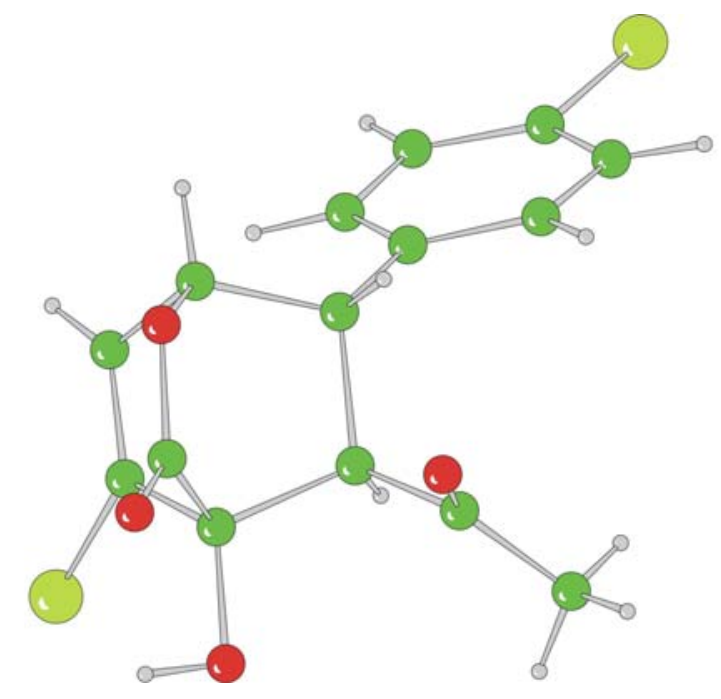

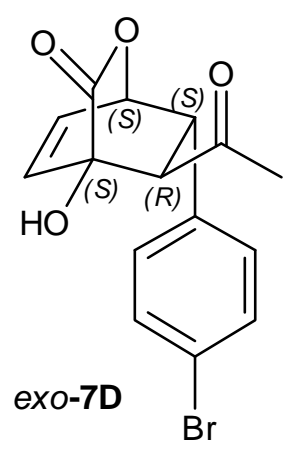

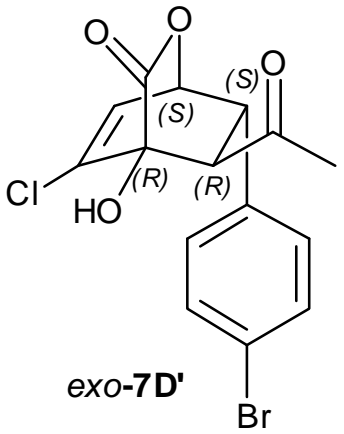

S-Figure 4. X-ray structure of 7D' 


\subsection{Assignment of the relative configuration of other D-A adducts 7 by ${ }^{1} \mathrm{H}$ NMR}

analysis.

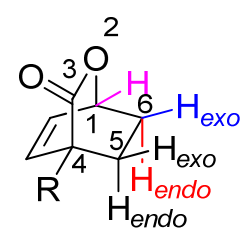

Reported Coupling Constant $\mathrm{H}_{1}-\mathrm{H}_{6}$ endo $=1.1-1.5 \mathrm{~Hz}$ $\mathrm{H}_{1}-\mathrm{H}_{6 \text { exo }}=2.0-4.5 \mathrm{~Hz}$

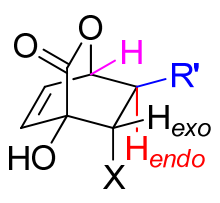

anti-endo

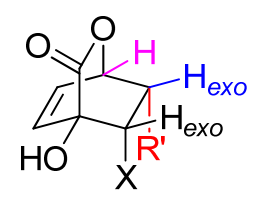

syn-endo

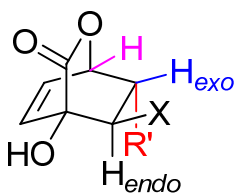

anti-exo

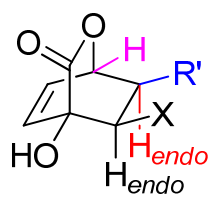

syn-exo

As described by Posner, ${ }^{11} \mathrm{H}_{6 e n d o}$ and $\mathrm{H}_{6 e x o}$ could be assigned by their respective coupling constant with $\mathrm{H} 1: \mathrm{H}_{1}-\mathrm{H}_{6 e n d o}=1.1-1.5 \mathrm{~Hz}, \mathrm{H}_{1}-\mathrm{H}_{6 e x o}=2.0-4.5 \mathrm{~Hz}$. After $\mathrm{H}_{6 e n d o}$ and/or $\mathrm{H}_{6 \text { exo }}$ were assigned by this method, the configuration of $\mathrm{C}_{5}$ relative to $\mathrm{C}_{6}$ was assigned by $\mathrm{H}_{5}-\mathrm{H}_{6}$ coupling constant (syn, 9-10 Hz; anti, 4-6 Hz). Consequently, the endo or exo configuration could be assigned for the cycloadducts 7 . 


\section{References:}

(1) Mathias, L. J.; Overberger, C. G. Synth. Comm. 1975, 5, 461.

(2) Wang, Y.; Li, H.; Wang, Y.-Q.; Liu, Y.; Foxman, B. M.; Deng, L. J. Am. Chem. Soc. 2007, 129, 6364.

(3) Komiyama, T.; Takaguchi, Y.; Gubaidullin, A. T.; Mamedov, V. A.; Litvinov, I. A.; Tsuboi, S. Tetrahedron 2005, 61, 2541.

(4) Paul, S.; Gupta, M. Synth. Comm. 2005, 35, 213.

(5) Grant, T. N.; West, F. G. J. Am. Chem. Soc. 2006, 128, 9348

(6) Wakamatsu, H.; Blechert, S. Angew. Chem. Int. Ed. 2002, 41, 2403.

(7) Barbry, D.; Faven, C.; Ajana, A. Synth. Comm. 1993, 23, 2647.

(8) (a) Li, H.; Wang, Y.; Tang, L.; Deng, L. J. Am. Chem. Soc. 2004, 126, 9906.

(9) (a) Vakulya, B.; Varga, S.; Csámpai, A.; Soós, T. Org Lett. 2005, 7, 1967. (b) Li, B.; Jiang, L.; Liu, M.; Chen, Y.; Ding, L.; Wu, Y. Synlett 2005, 4, 603.

(10) Marcelli, T.; Haas, R. N. S. v. d.; Maarseveen, J. H. v.; Hiemstra, H. Angew. Chem. Int. Ed. 2006, 45, 929.

(11) Afarinkia, K.; Vinader, V.; Nelson, T. D.; Posner, G. H. Tetrahedron 1992, 48, 9111 and references therein. 

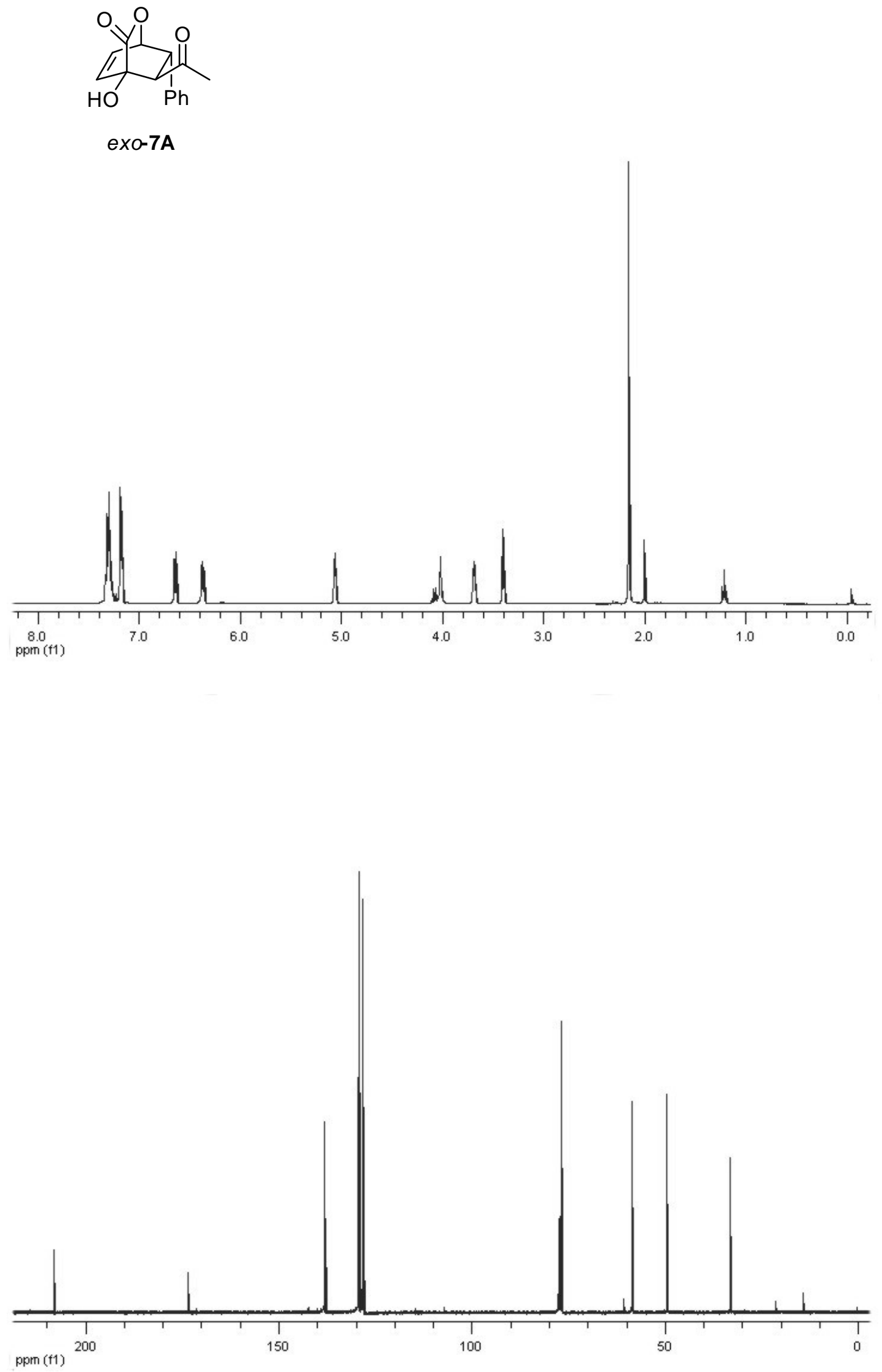

S 21 
HPLC, Chiralcel OD, hexanes: isopropanol $=70: 30,1.0 \mathrm{~mL} / \mathrm{min}, \lambda=220 \mathrm{~nm}$.

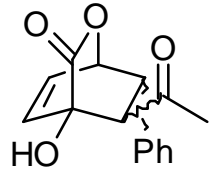

racemic-7A

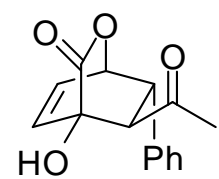

exo-7A, $98 \%$ ee obtained from QD-4 catalyzed reaction

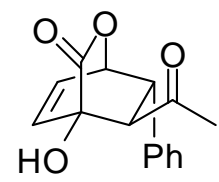

ent-exo-7A, $96 \%$ ee obtained from Q-4 catalyzed reaction
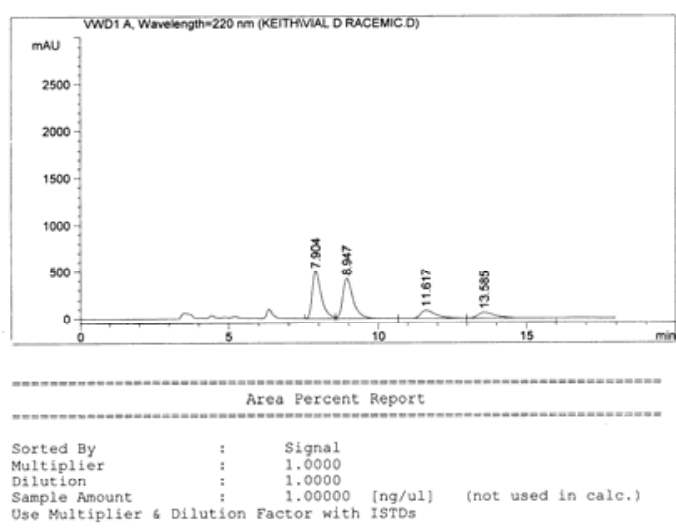

Signal 1: WwD1 A, wavelength-220 nm

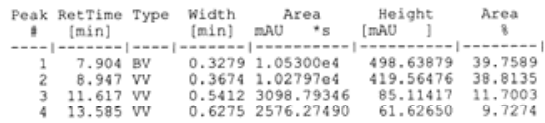
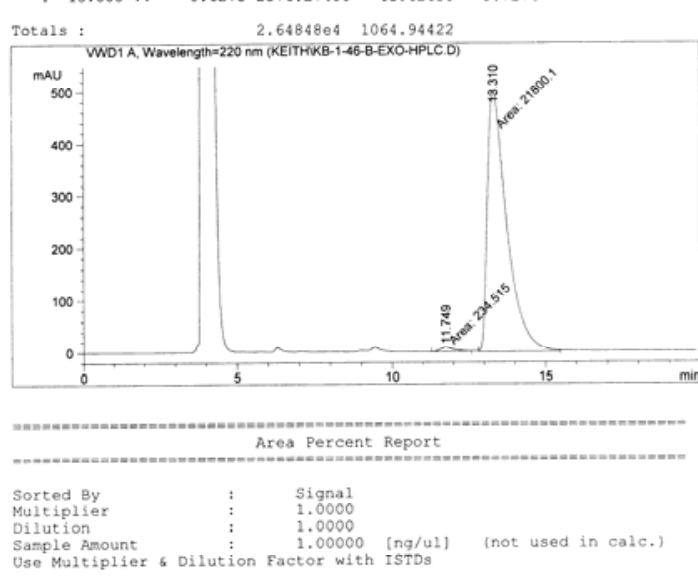

Sample Amount
Use Multipliser s. Dilution Factor with ISTDs

Signal 1: VWD1 $\mathrm{A}$, wavelength $=220 \mathrm{~nm}$

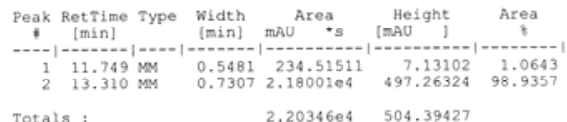

$\begin{array}{lll}\text { Totals : } & 2.20346 \mathrm{e} & 504.39427\end{array}$
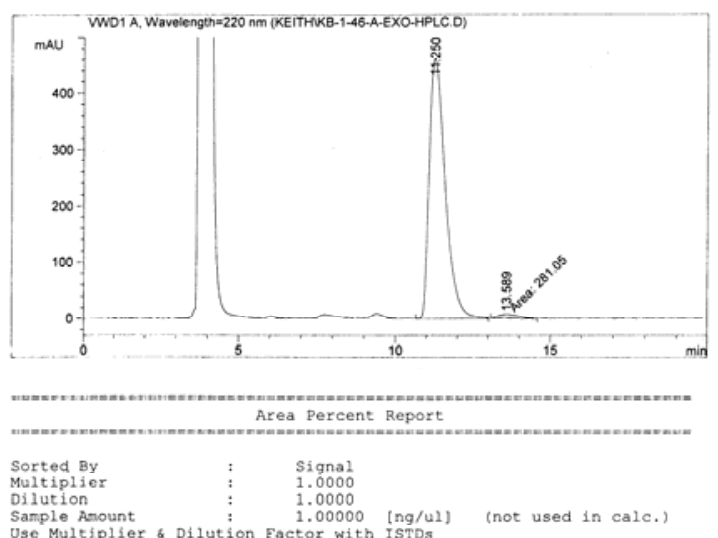

Sample Amount
Use Multiplier \& Dilution Factor with ISTDs

Signal 1: WWD1 A, wavelength=220 $\mathrm{nm}$

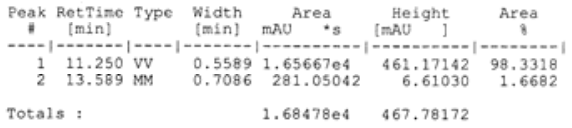



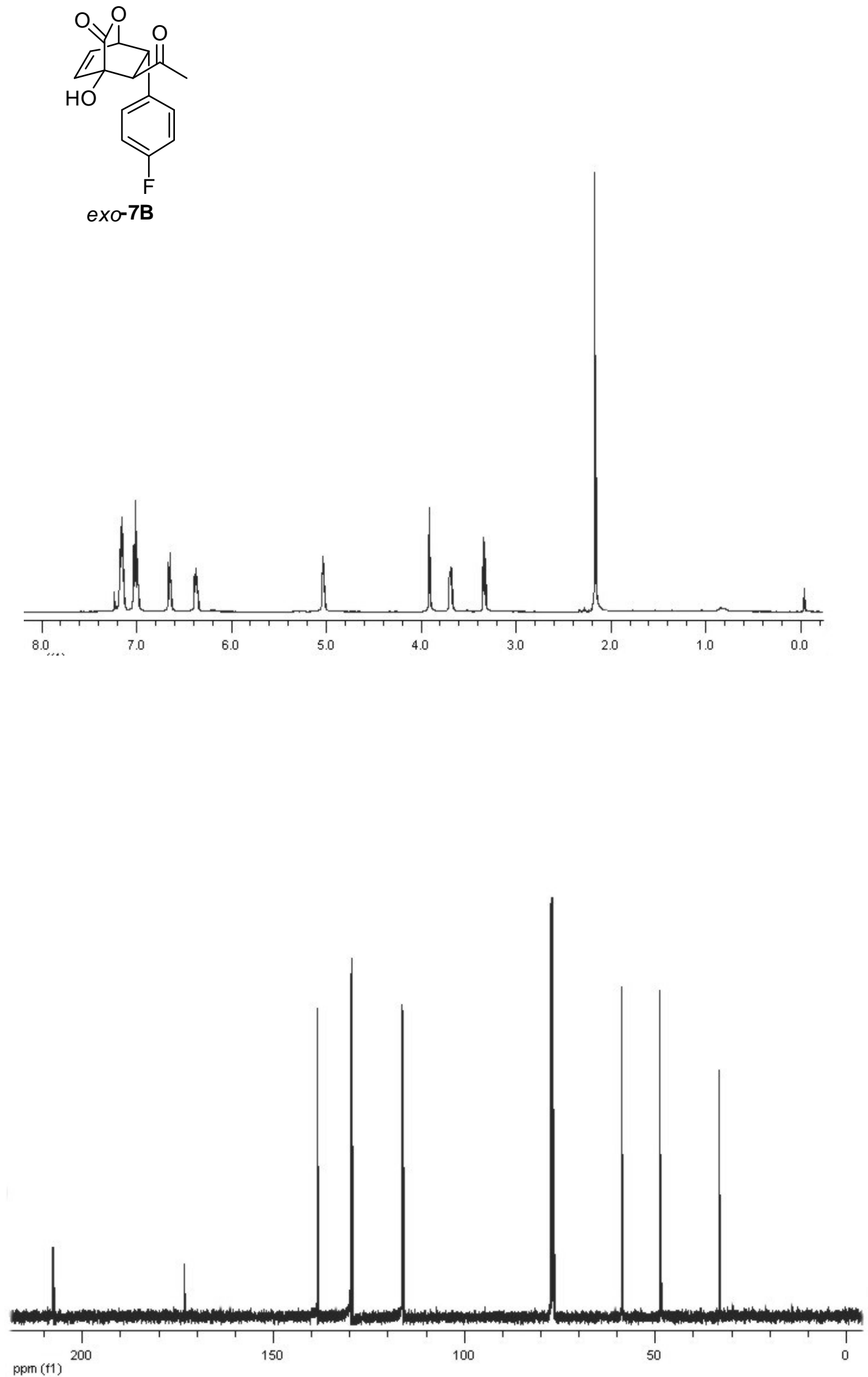

S 23 
HPLC, Chiralpak AD, hexanes: isopropanol $=70: 30,0.75 \mathrm{~mL} / \mathrm{min}, \lambda=220 \mathrm{~nm}$.<smiles>O=C(CC1(O)C=CC2(O)CC1CC2=O)c1ccc(F)cc1</smiles>

racemic-7B
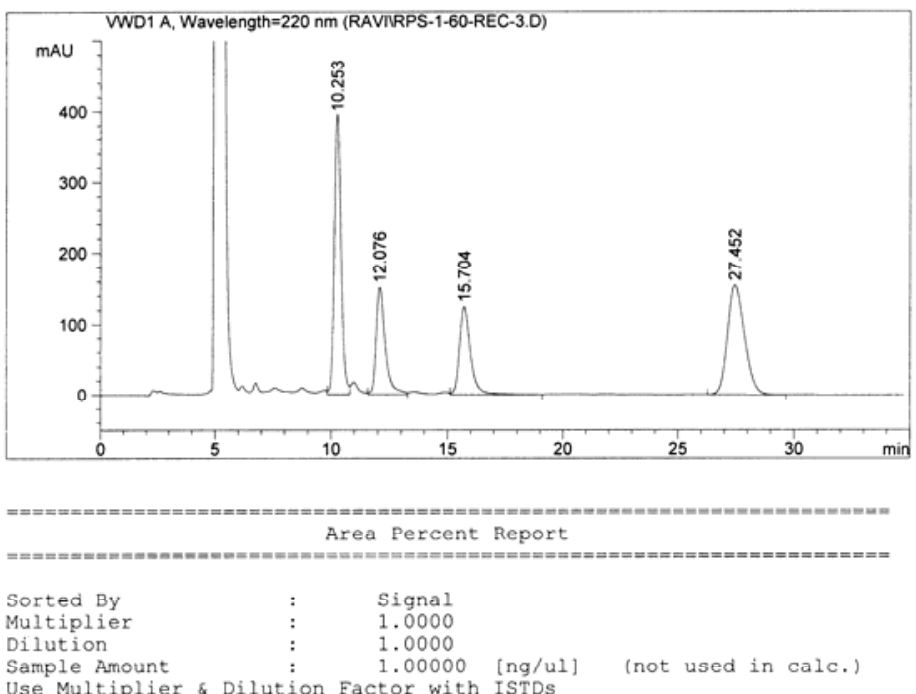

Use Multiplier \& Dilution Factor with ISTDs

Signal 1: VWD1 A, Wavelength $=220 \mathrm{~nm}$

\begin{tabular}{|c|c|c|c|c|c|c|}
\hline Peak & RetTime & Type & Width & Area & Height & Area \\
\hline \# & [min] & & {$[\mathrm{min}]$} & mAU & {$[\mathrm{mAU}$} & 8 \\
\hline 1 & 10.253 & vV & 0.3044 & 7895.60791 & 392.82587 & 32.9381 \\
\hline 2 & 12.076 & vV & 0.4101 & 4097.28711 & 150.85579 & 17.0926 \\
\hline 3 & 15.704 & vV & 0.4901 & 4097.79736 & 124.08435 & 17.0948 \\
\hline 4 & 27.452 & vv & 0.7933 & 7880.38281 & 154.43808 & 32.8745 \\
\hline
\end{tabular}

Totals :

$2.39711 \mathrm{e} 4 \quad 822.20409$

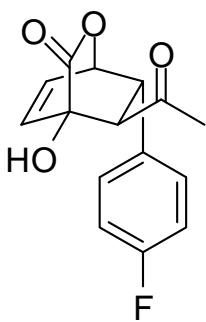

exo-7B, $98 \%$ ee obtained from QD-4 catalyzed reaction
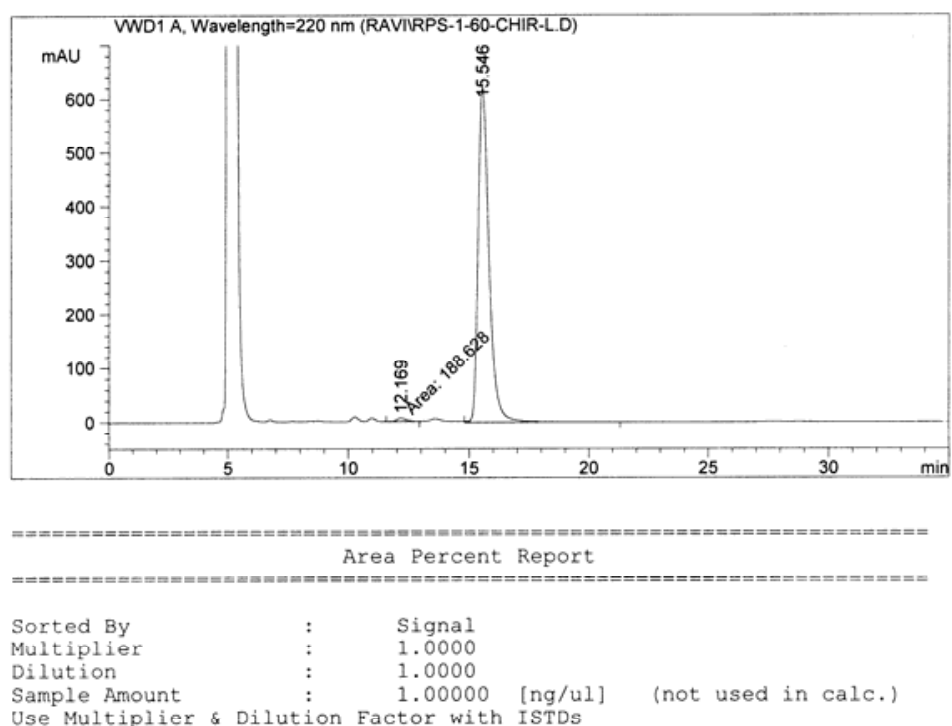

Signal 1: VWD1 A, Wavelength=220 nm

\begin{tabular}{|c|c|c|c|c|c|c|}
\hline Peak & RetTime & Type & Width & Area & Height & Area \\
\hline & {$[\mathrm{min}]$} & & [min] & $\mathrm{mAU}$ & [mAU & 8 \\
\hline 2 & $\begin{array}{l}12.169 \\
15.546\end{array}$ & $\begin{array}{l}\text { MM } \\
\text { VV }\end{array}$ & $\begin{array}{l}0.4939 \\
0.4762\end{array}$ & $\begin{array}{r}188.62845 \\
1.96994 \mathrm{e} 4\end{array}$ & $\begin{array}{r}6.36523 \\
618.89630\end{array}$ & $\begin{array}{r}0.9485 \\
99.0515\end{array}$ \\
\hline
\end{tabular}

Totals :

$1.98880 \mathrm{e} 4 \quad 625.2615$ 

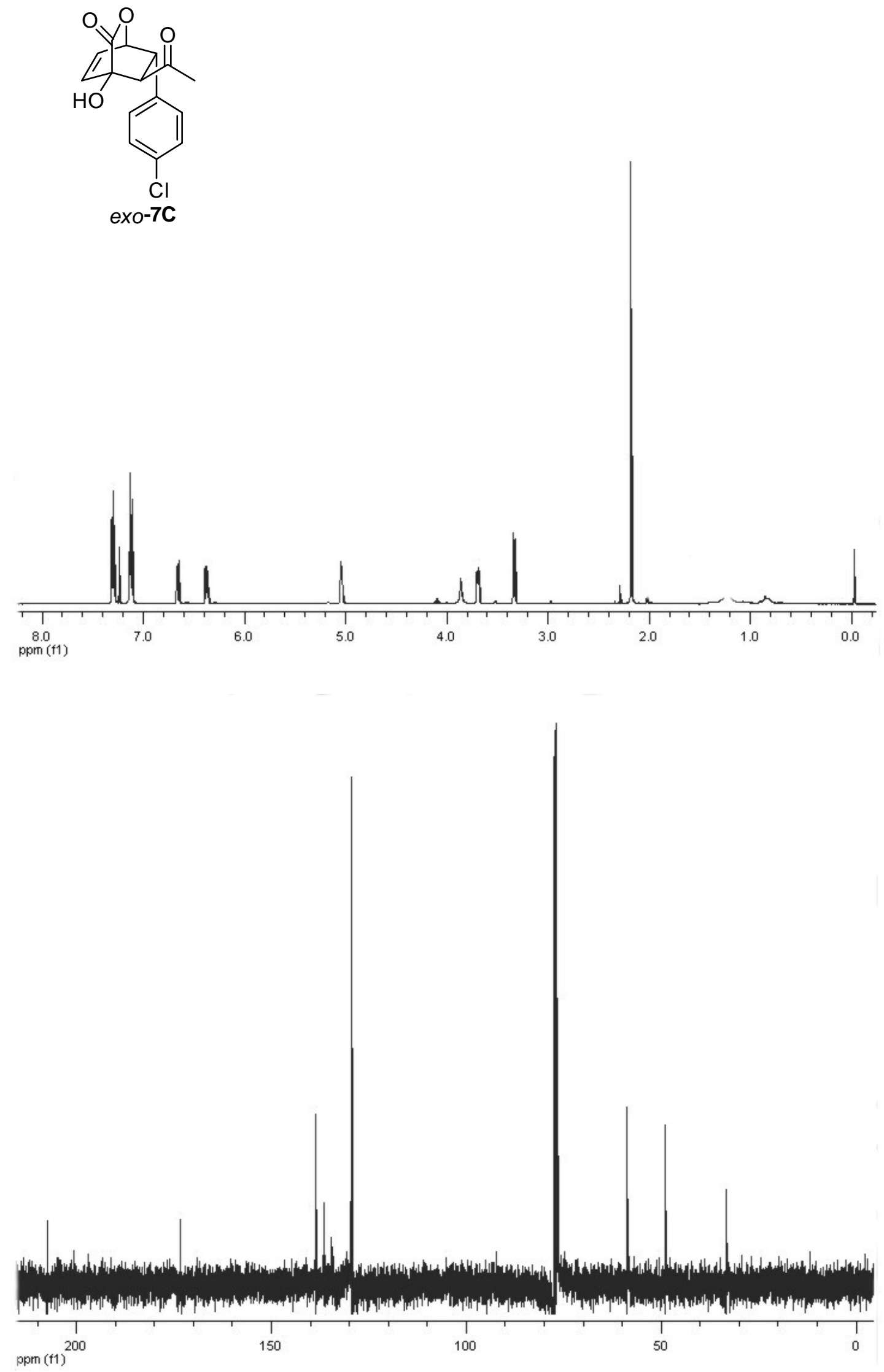

S 25 
HPLC, Chiralpak AD, hexanes: isopropanol $=85: 15,1.0 \mathrm{~mL} / \mathrm{min}, \lambda=220 \mathrm{~nm}$.

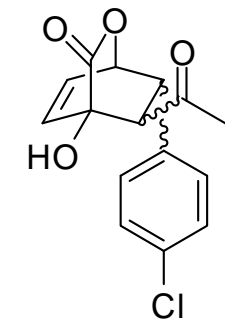

racemic-7C<smiles>CC(=O)CC1(O)C=CC(=O)C(O)(C(=O)O)C1=O</smiles>

exo-7C, $98 \%$ ee obtained from QD-4 catalyzed reaction<smiles>CC(=O)CC1(O)C=CC2(O)OC(=O)CC1(O)O2</smiles>

ent-exo-7C, $96 \%$ ee obtained from Q-4 catalyzed reaction
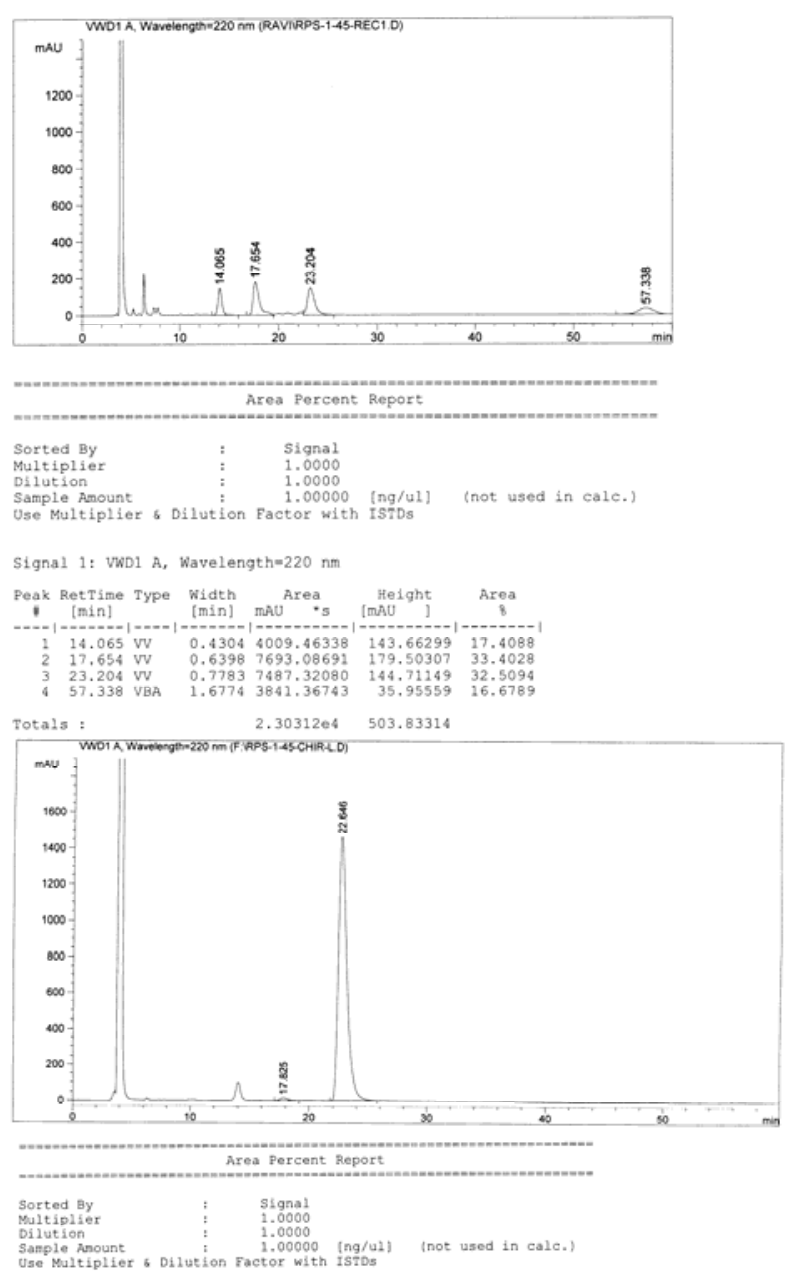

Signal 1: WwD1 $\mathrm{A}$, wavelength-220 $\mathrm{nm}$

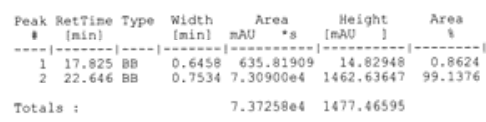
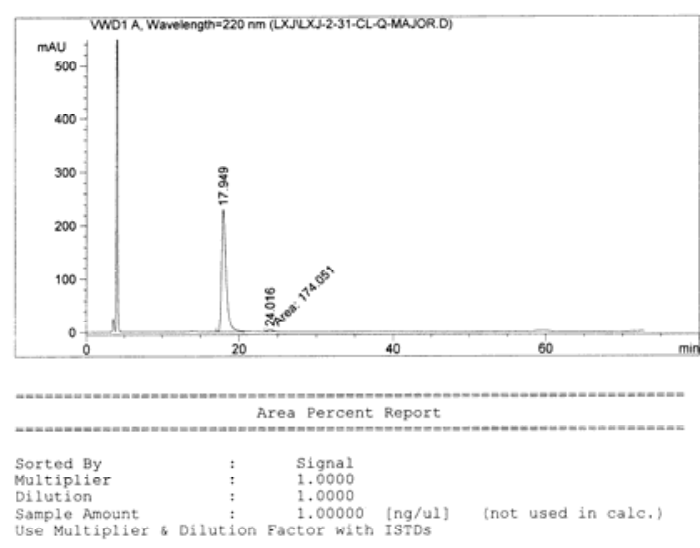

Signal 1: WWD1 $A$, wavelength $=220 \mathrm{~nm}$

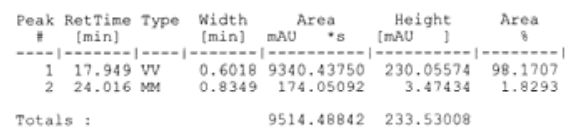




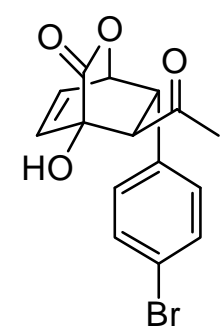

exo-7D
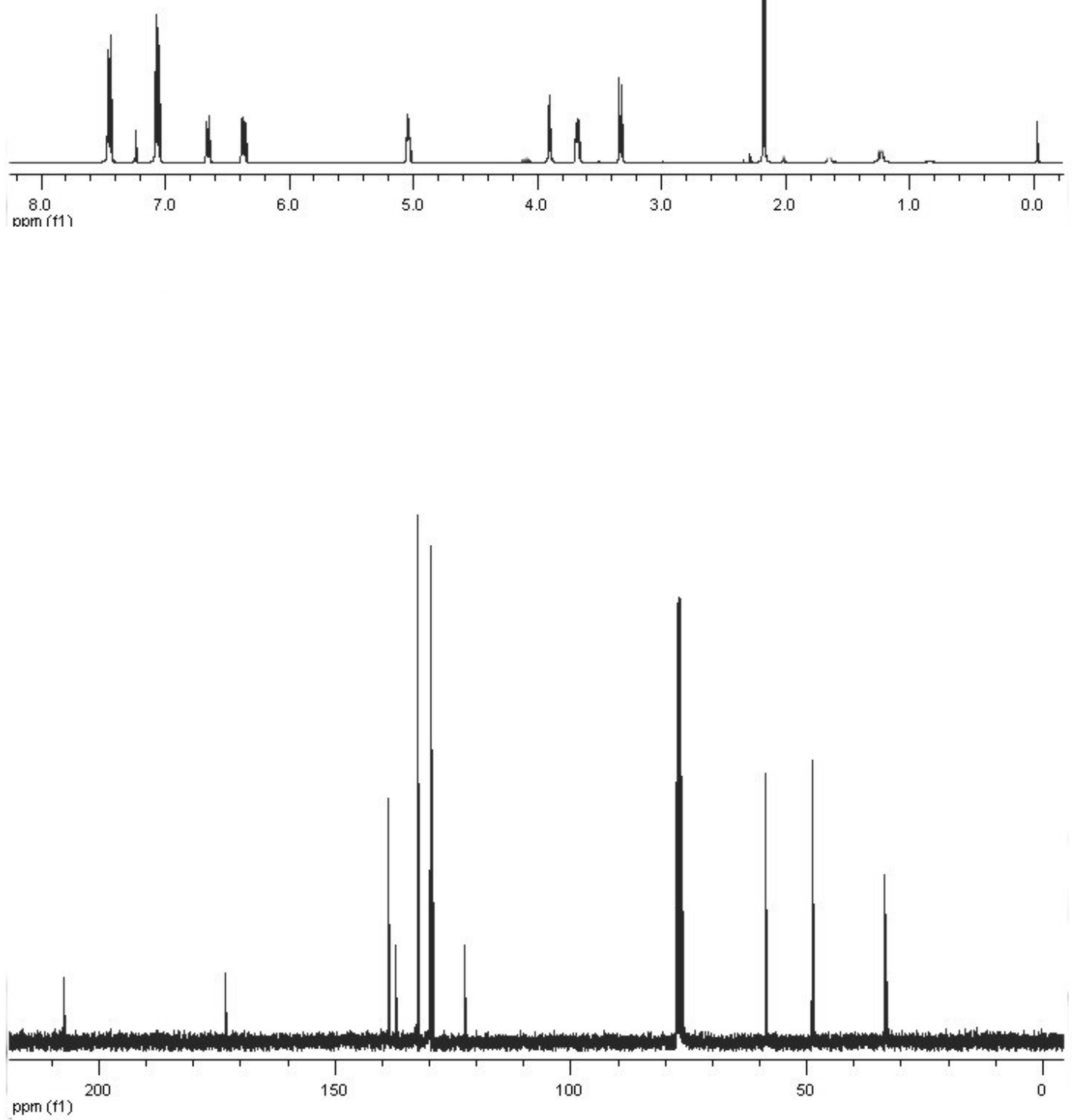

S 27 
HPLC, Chiralpak AD, hexanes: isopropanol $=80: 20,1.0 \mathrm{~mL} / \mathrm{min}, \lambda=220 \mathrm{~nm}$.<smiles>CC(=O)CC1(O)C=CC(=O)OC1(O)c1ccc(Br)cc1</smiles>

racemic-7D

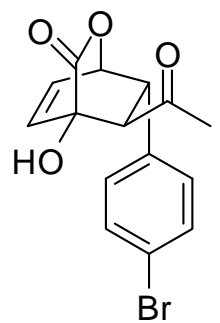

exo-7D, $97 \%$ ee obtained from QD-4 catalyzed reaction<smiles>CC(=O)CC1(O)C=CC2(O)OC(=O)CC1(O)O2</smiles>

ent- exo-7D, $97 \%$ ee obtained from Q-4 catalyzed reaction
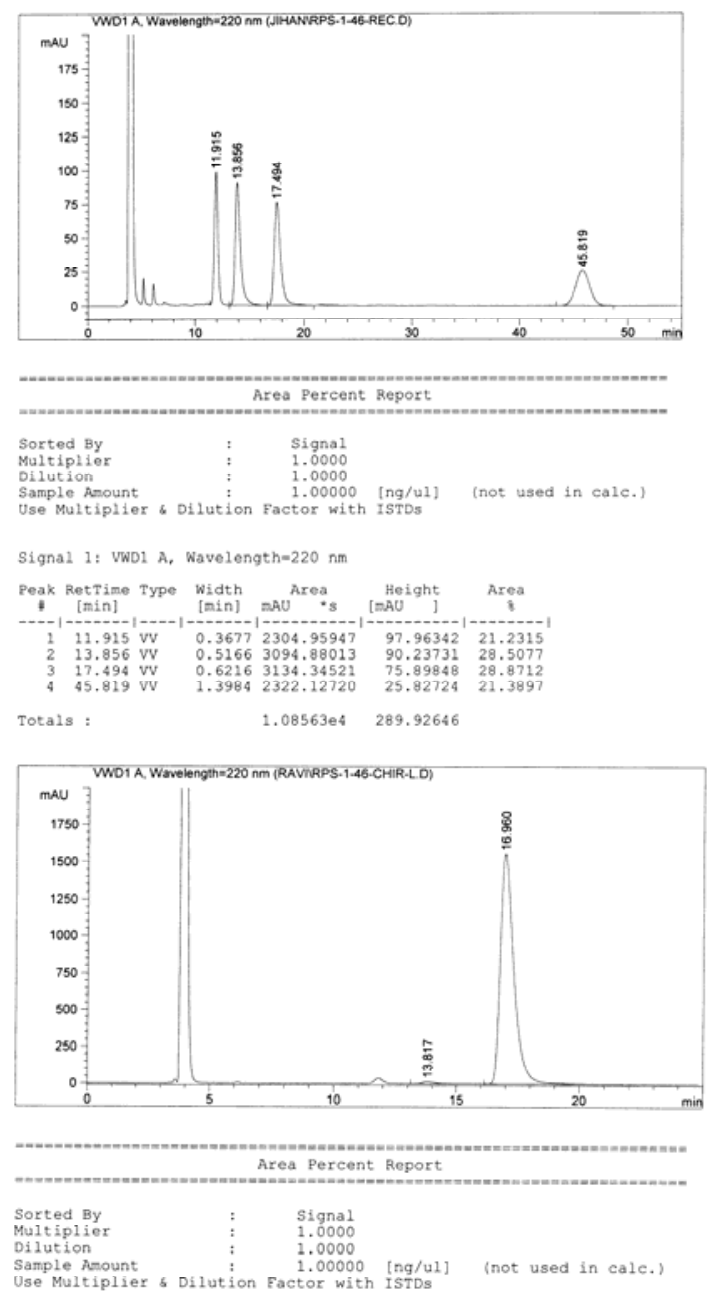

Signal 1: VWD1 $A$, wavelength $=220 \mathrm{~nm}$

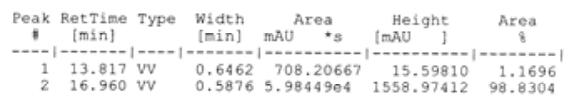
Totals : $\quad 6.05531 \mathrm{e} 4 \quad 1574.57222$
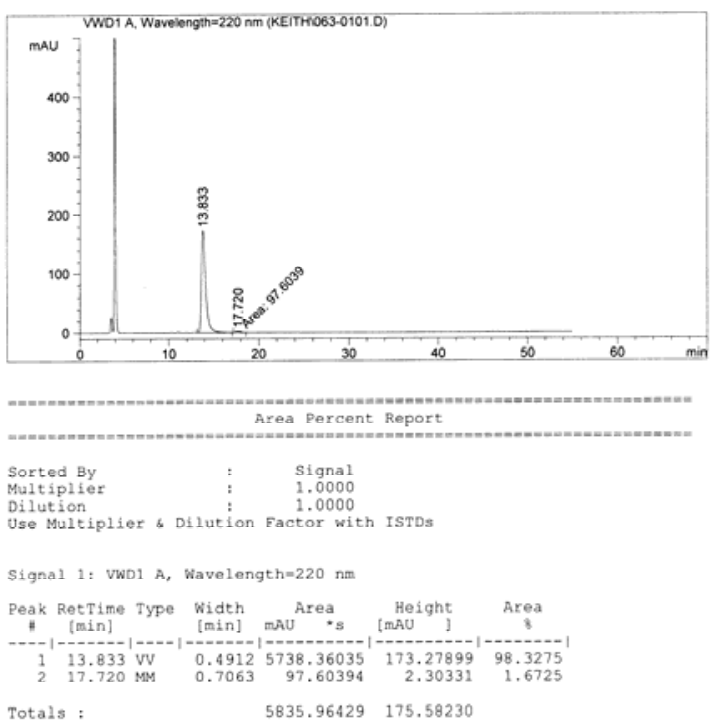


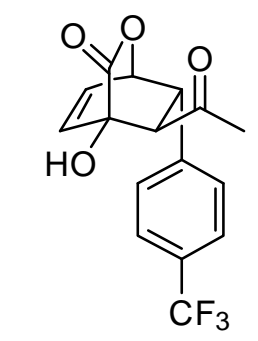

exo-7E
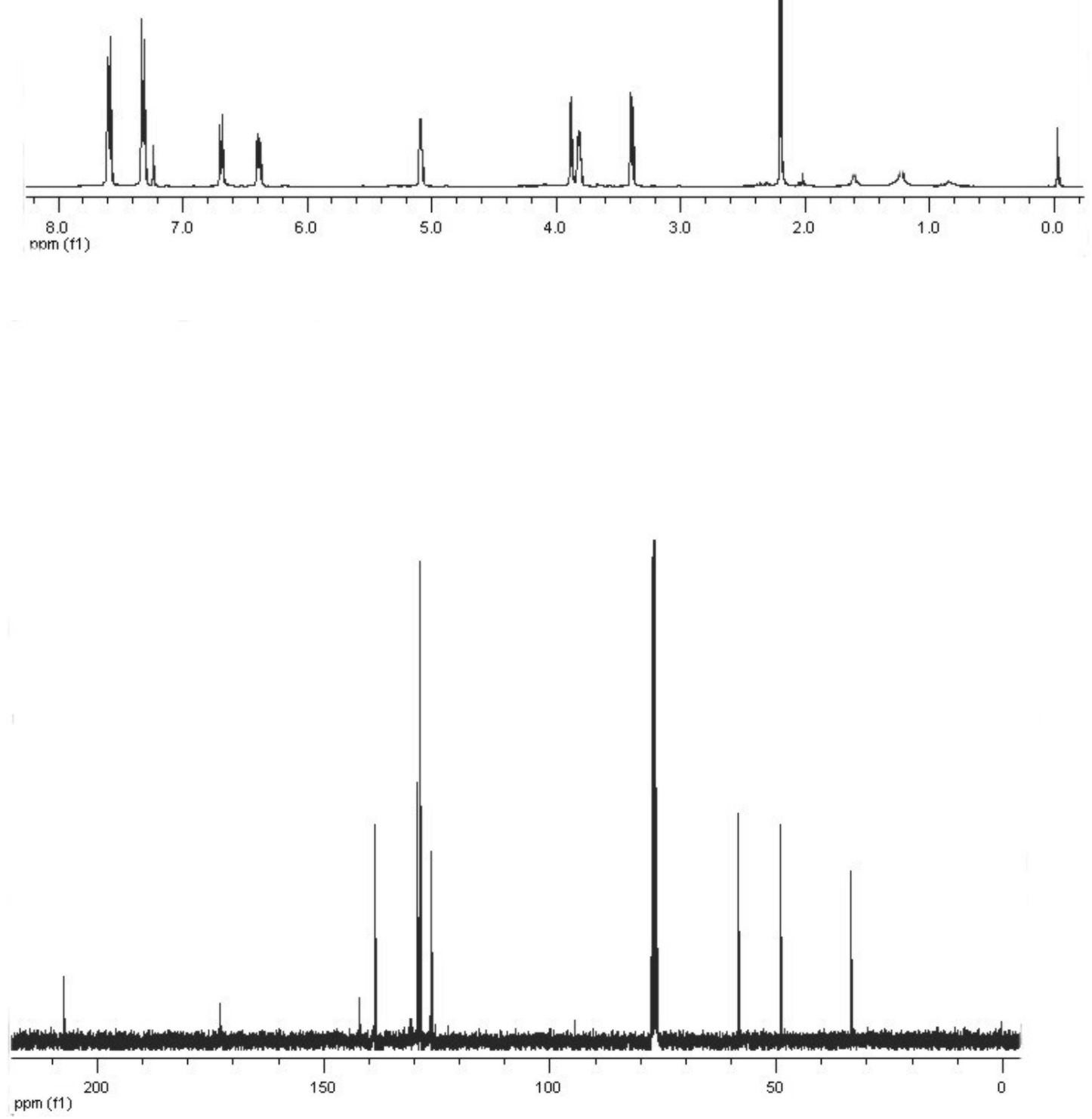

S 29 
HPLC, Chiralpak AD, hexanes: isopropanol $=90: 10,1.0 \mathrm{~mL} / \mathrm{min}, \lambda=220 \mathrm{~nm}$.

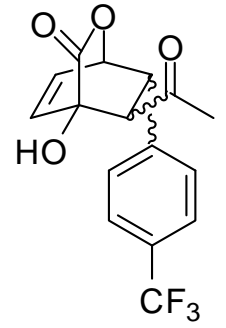

racemic- $\mathbf{7 E}$

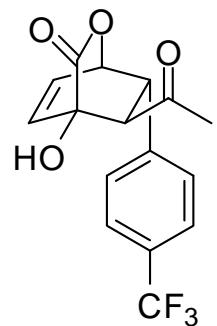

exo-7E, $97 \%$ ee obtained from QD-4 catalyzed reaction
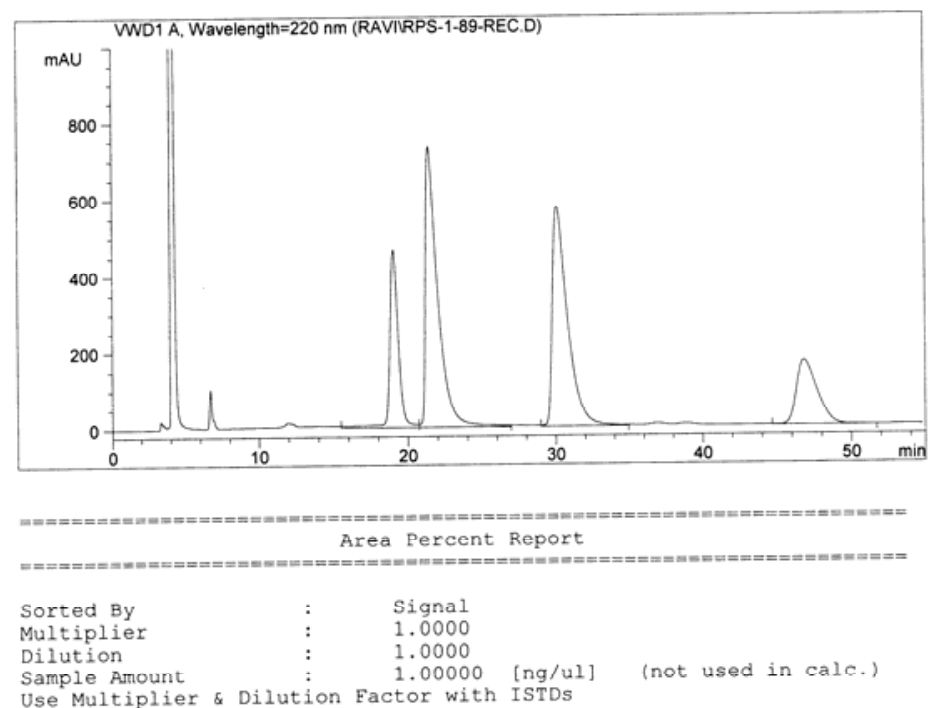

Use Multiplier \& Dilution Factor with ISTDs

Signal 1: VWD1 $\mathrm{A}$, Wavelengt $\mathrm{h}=220 \mathrm{~nm}$

\begin{tabular}{|c|c|c|c|c|c|c|}
\hline ak & RetTime & Type & width & Area & Height & Area \\
\hline \# & [min] & & & $\mathrm{mAU}$ & {$[\mathrm{mAU}$} & 8 \\
\hline 1 & 19.046 & vV & 0.6100 & $1.87716 \mathrm{e} 4$ & 465.70090 & 15.7695 \\
\hline 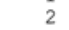 & 21 & vV & 0.8 & 4.23812 & 732.13 & 032 \\
\hline 3 & 30.107 & vV & 1.0661 & $4.16128 \mathrm{e} 4$ & 574.66 & 77 \\
\hline 4 & 46.825 & $\mathrm{vV}$ & 1.4653 & $1.62719 \mathrm{e} 4$ & 168.36356 & 13.6695 \\
\hline & & & & $1.19038 \mathrm{e} 5$ & 40.86176 & \\
\hline
\end{tabular}
$1.19038 \mathrm{e} 5 \quad 1940.86176$
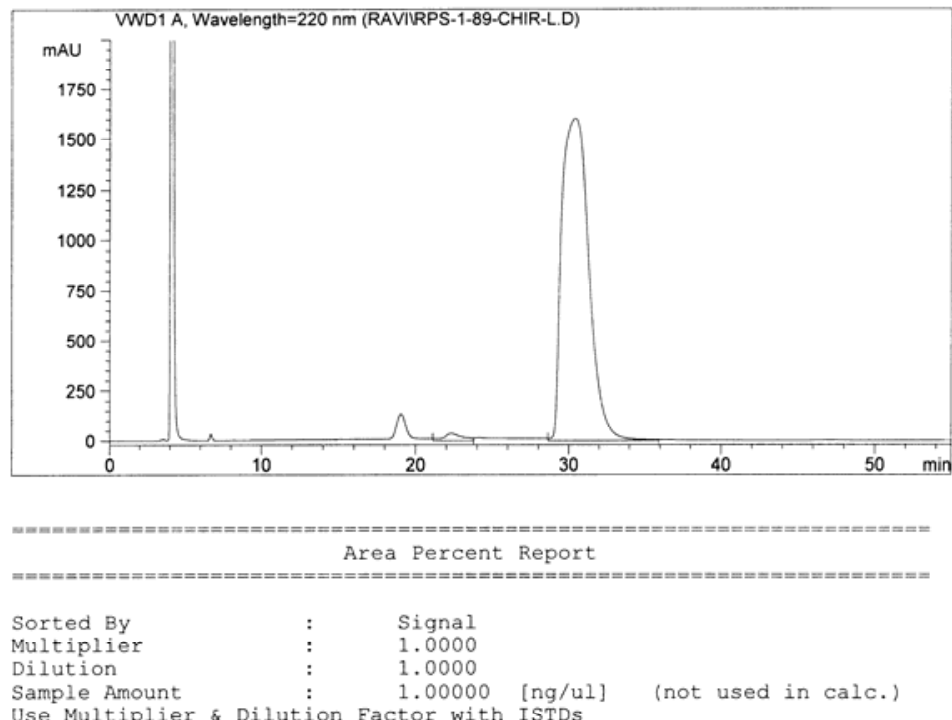

Use Multiplier \& Dilution Factor with ISTDS

Signal 1: VWD1 A, Wavelengt $h=220 \mathrm{~nm}$

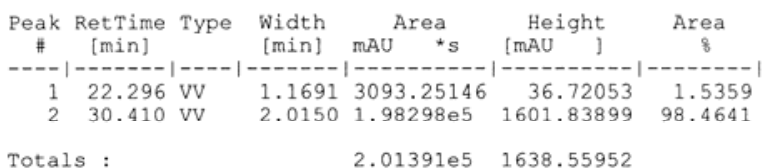

Totals : $\quad 2.01391 e 5 \quad 1638.55952$ 

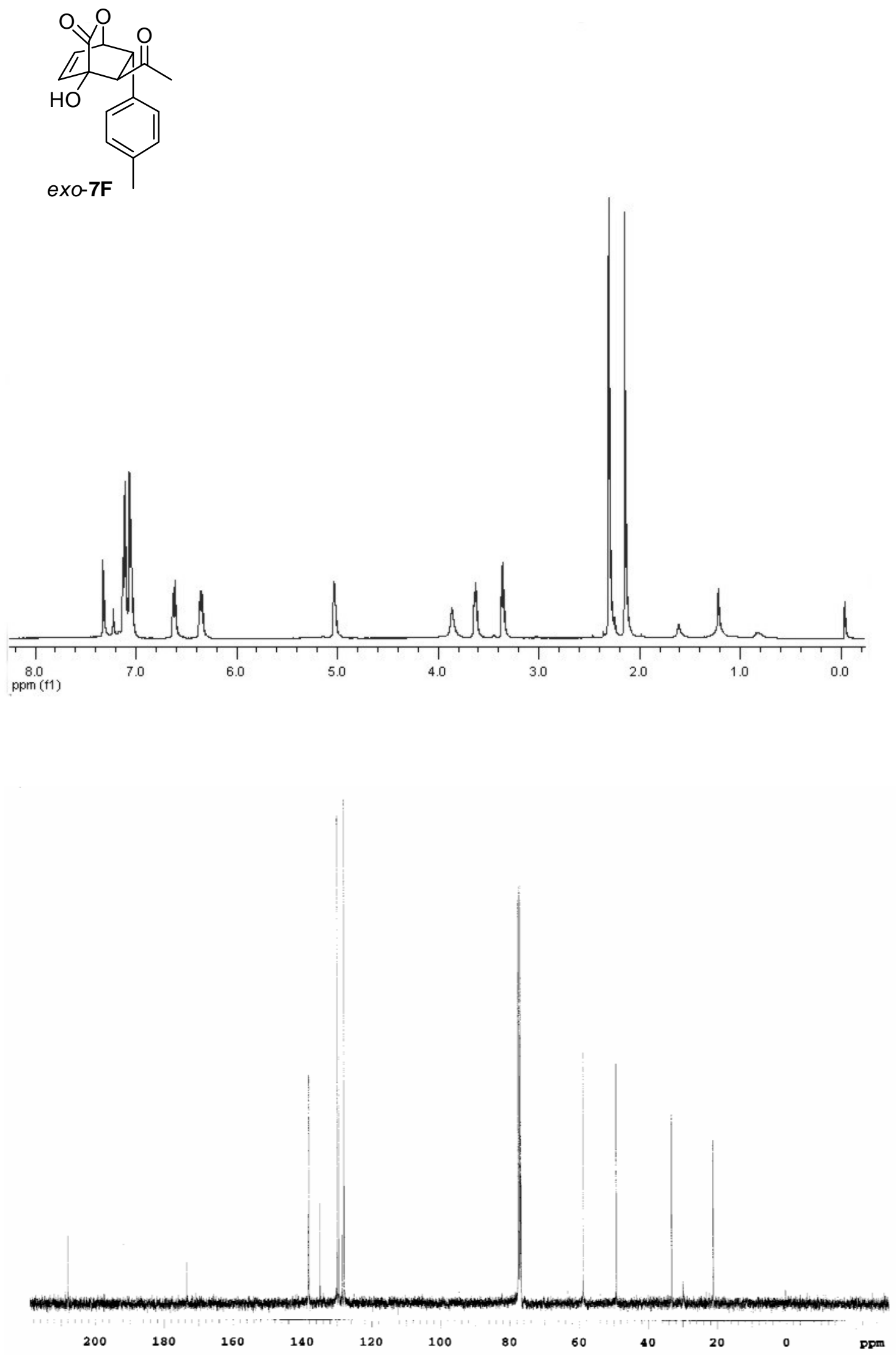

S 31 
HPLC, Chiralpak AD, hexanes: isopropanol $=80: 20,1.0 \mathrm{~mL} / \mathrm{min}, \lambda=220 \mathrm{~nm}$

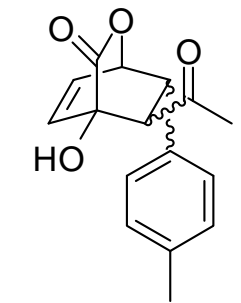

racemic-7F

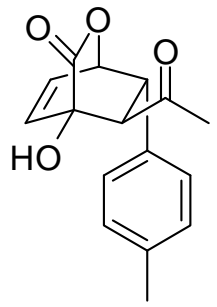

exo-7F, $97 \%$ ee obtained from QD-4 catalyzed reaction
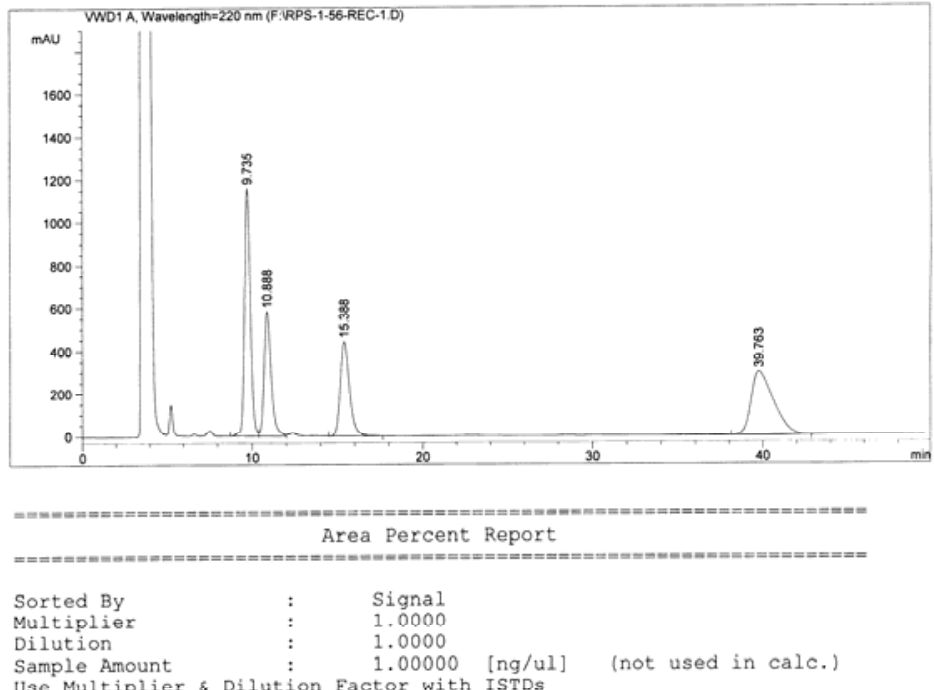

Use Multiplier \& Dilution Factor with ISTDs

Signal 1: VWD1 $\mathrm{A}$, Wavelength $=220 \mathrm{~nm}$

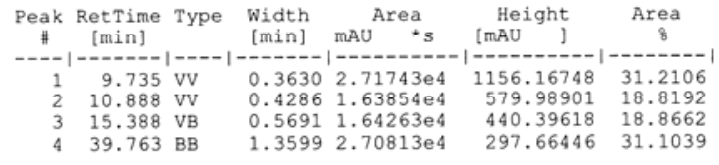

Totals :

8. $70673 \mathrm{e} 4 \quad 2474.21713$

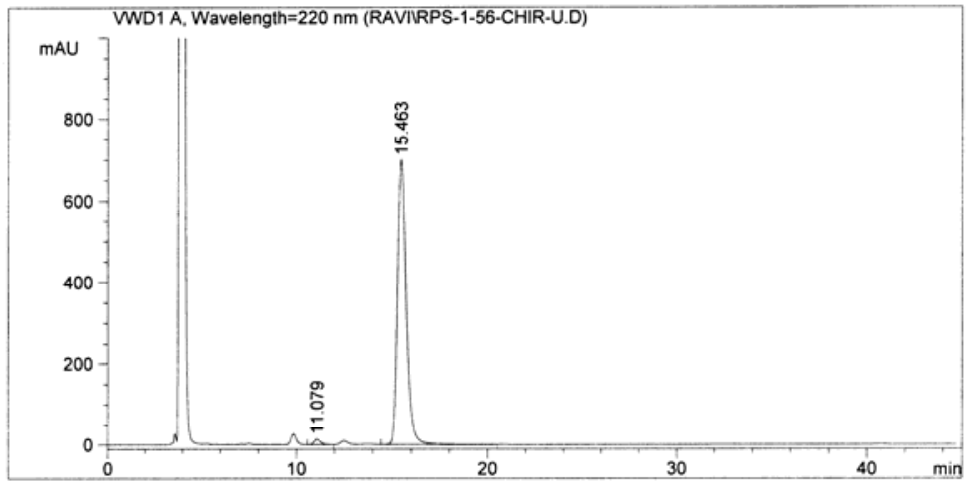

Area Percent Report

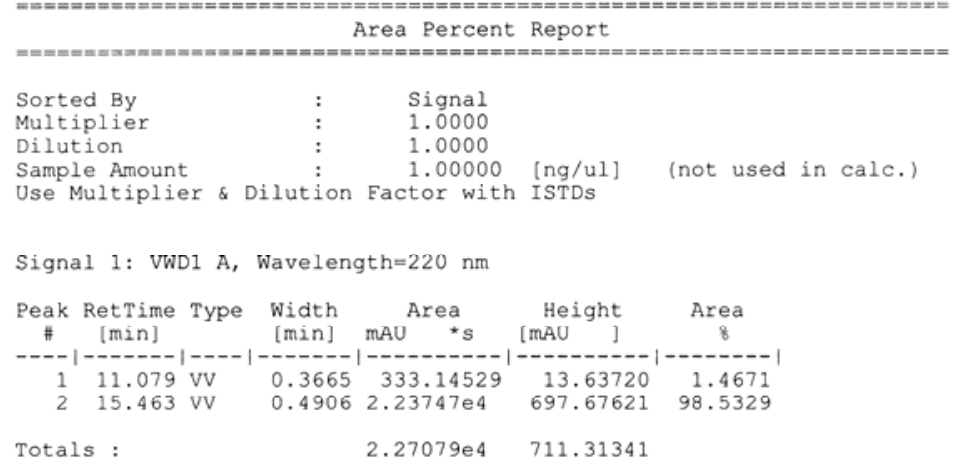



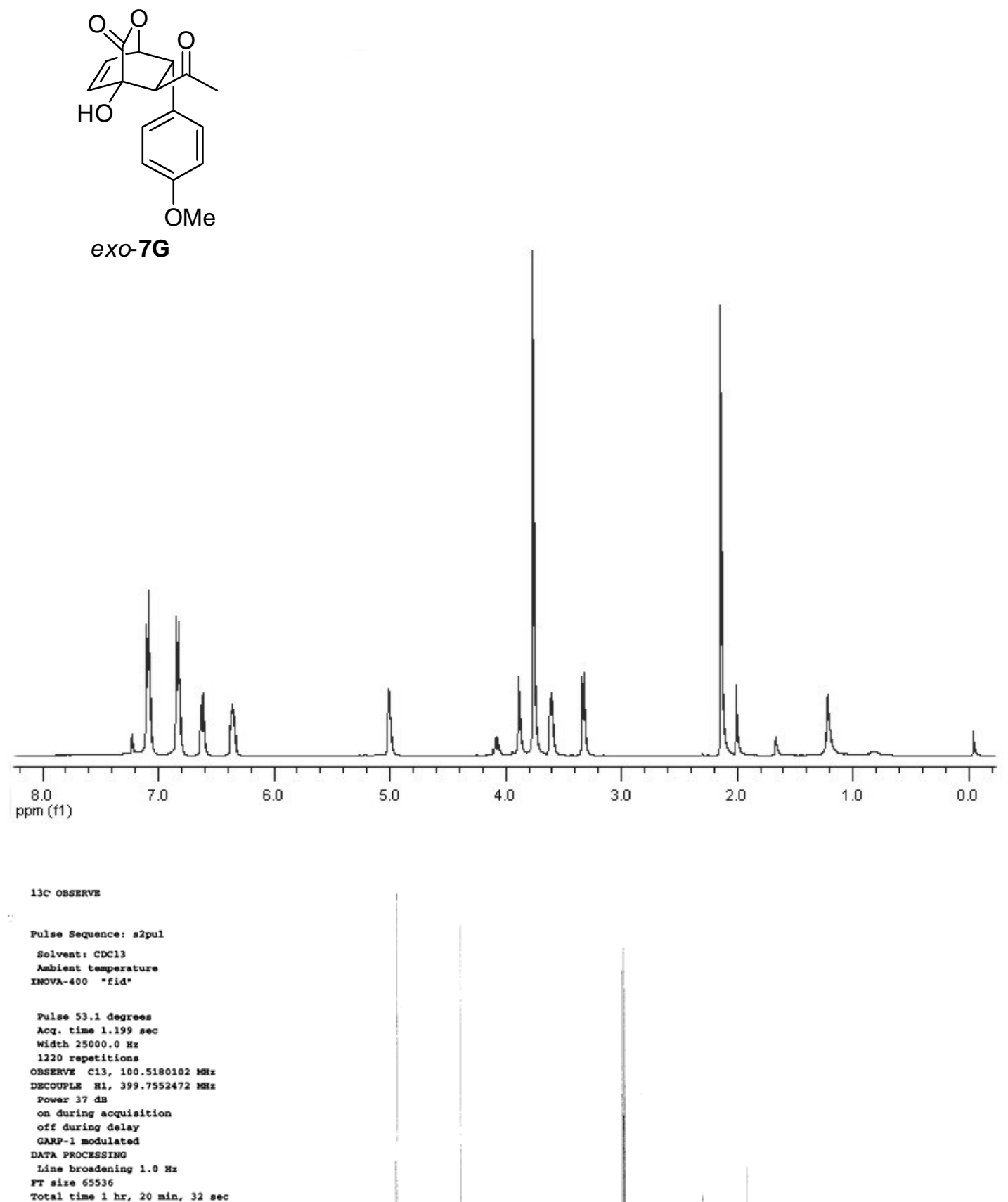
HPLC, Chiralpak AD, hexanes: isopropanol $=80: 20,0.75 \mathrm{~mL} / \mathrm{min}, \lambda=220 \mathrm{~nm}$

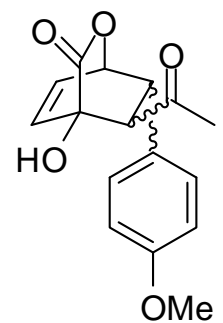

racemic-7G

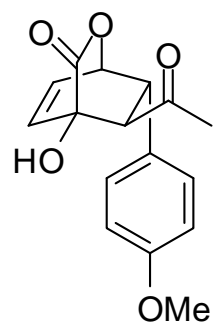

exo-7G, $97 \%$ ee obtained from QD-4 catalyzed reaction
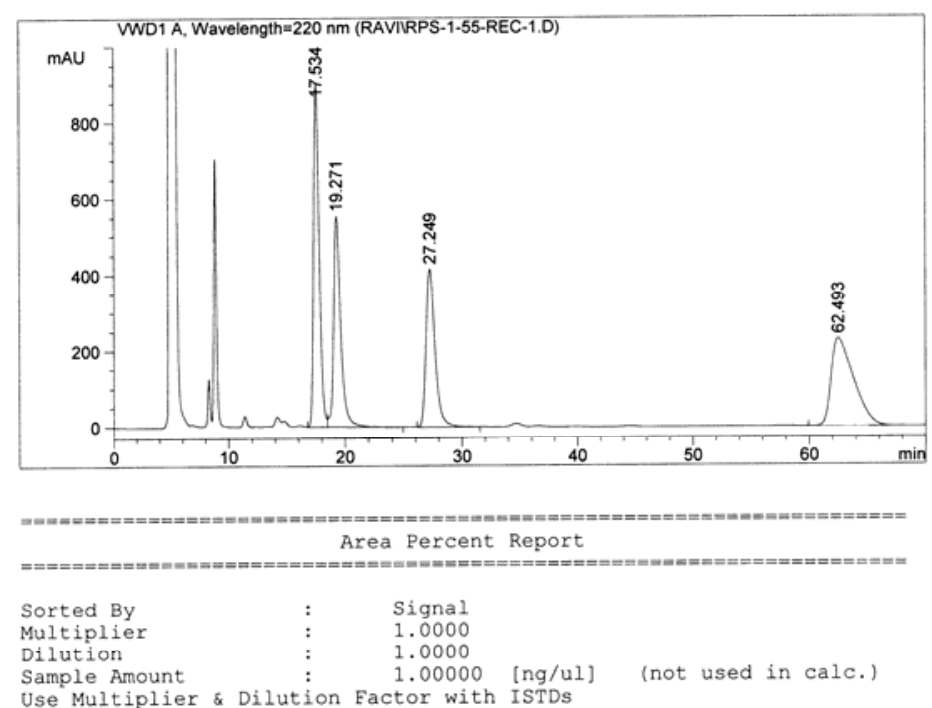

Use Multiplier \& Dilution Factor with ISTDS

Signal 1: VWD1 $\mathrm{A}$, Wavelengt $\mathrm{h}=220 \mathrm{~nm}$

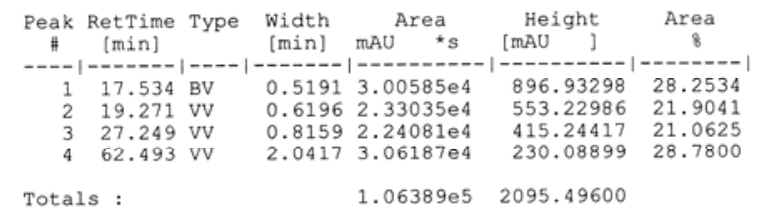

Totals : $1.06389 \mathrm{e} 5 \quad 2095.49600$
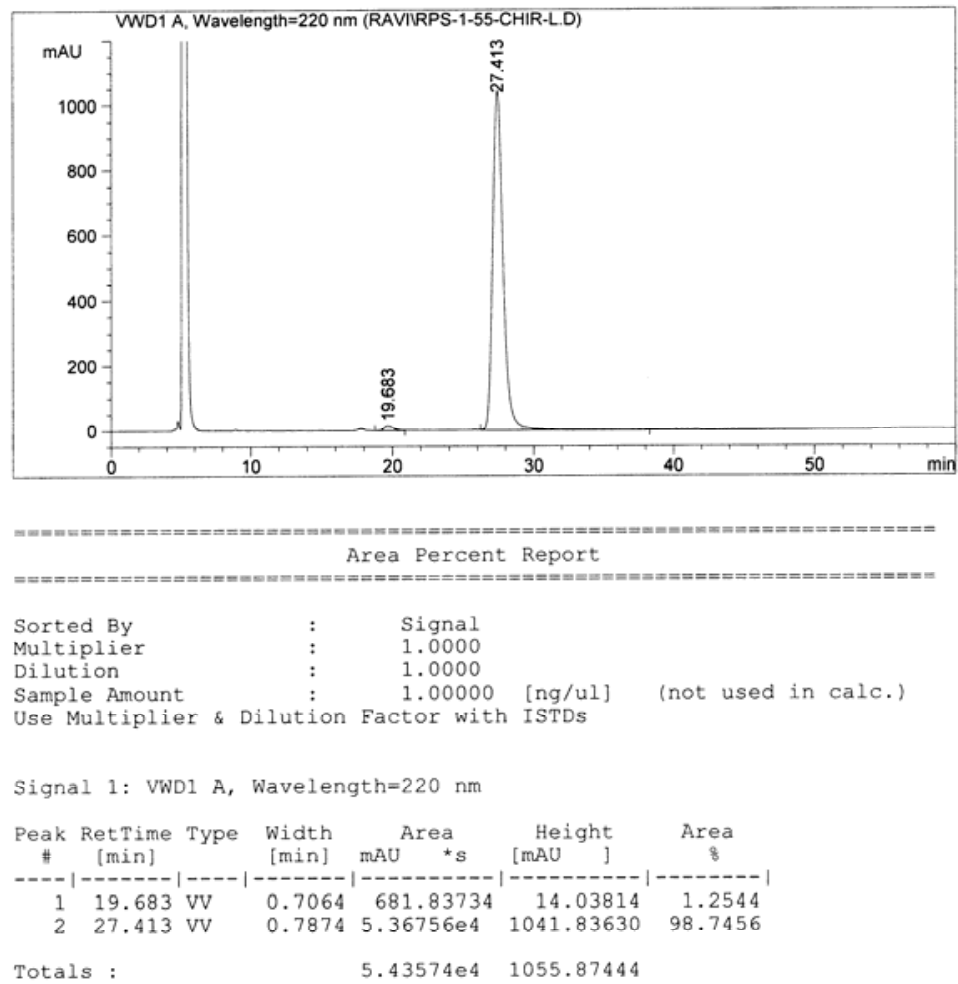


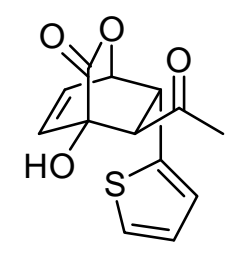

exo-7H
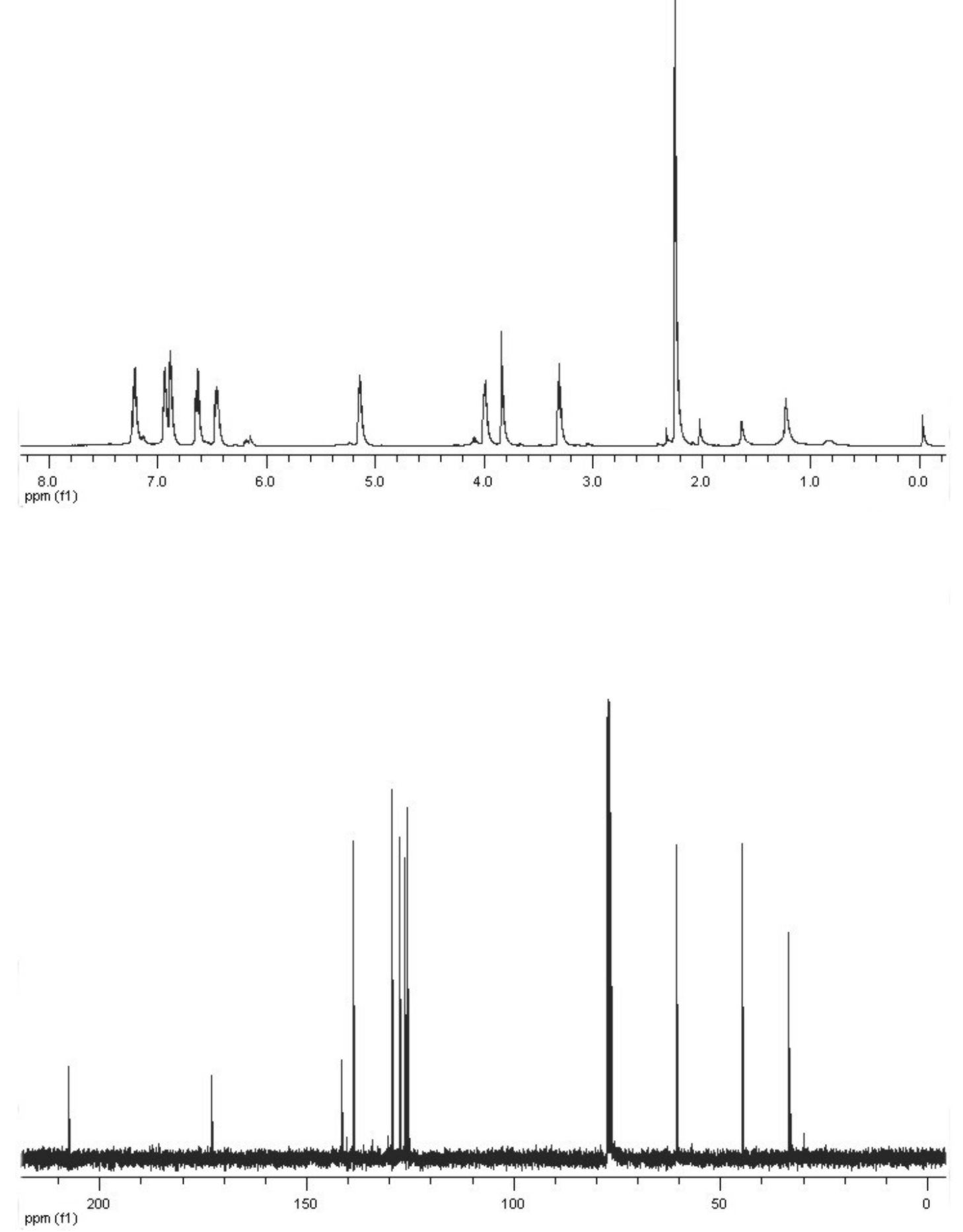
HPLC, Chiralpak AD + Chiralcel OJ, hexanes: isopropanol $=80: 20,1.0 \mathrm{~mL} / \mathrm{min}, \lambda=$ $220 \mathrm{~nm}$

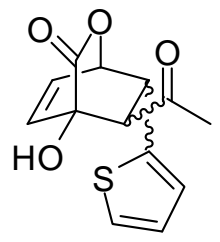

racemic-7H

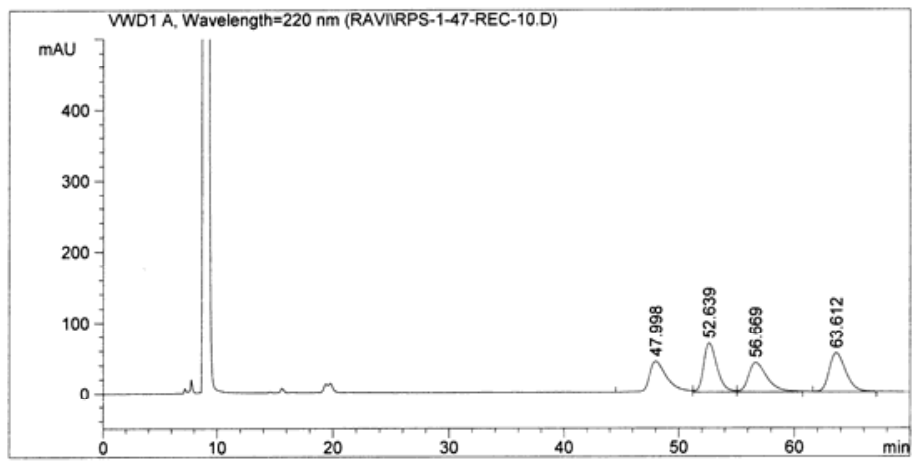

Area Percent Report

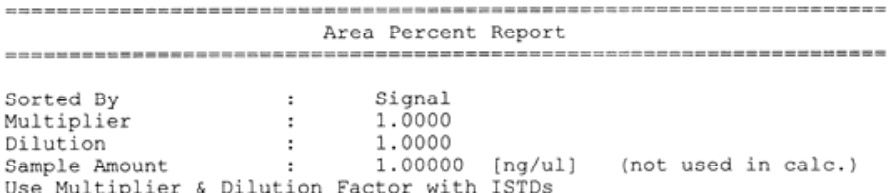

Use Multiplier \& Dilution Factor with ISTDs

Signal 1: VWD1 $A$, Wavelength $=220 \mathrm{~nm}$

\begin{tabular}{|c|c|c|c|c|c|c|}
\hline Peak & RetTime & Type & Width & Area & Height & Area \\
\hline \# & & & [min] & MAU & [MAU & 8 \\
\hline 1 & 47.998 & vV & 1.6008 & 4556.70605 & 43.29018 & 22.5642 \\
\hline 2 & 52.639 & vV & 1.24 & 553 & 68 & 27.4132 \\
\hline 3 & 56.669 & vV & 1.6646 & 4615.45264 & 41.30921 & 22.8551 \\
\hline 4 & 63.612 & vV & 1.5668 & 5486.29492 & 54.67057 & 27.1674 \\
\hline & & & & $2.01944 \mathrm{e} 4$ & 207.98 & \\
\hline
\end{tabular}

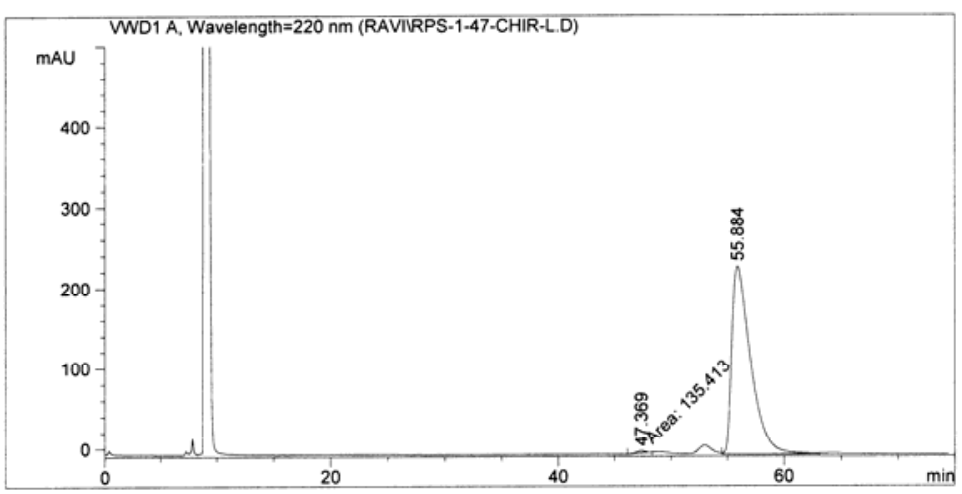

exo-7H, $99 \%$ ee obtained from QD-4 catalyzed reaction
Area Percent Report

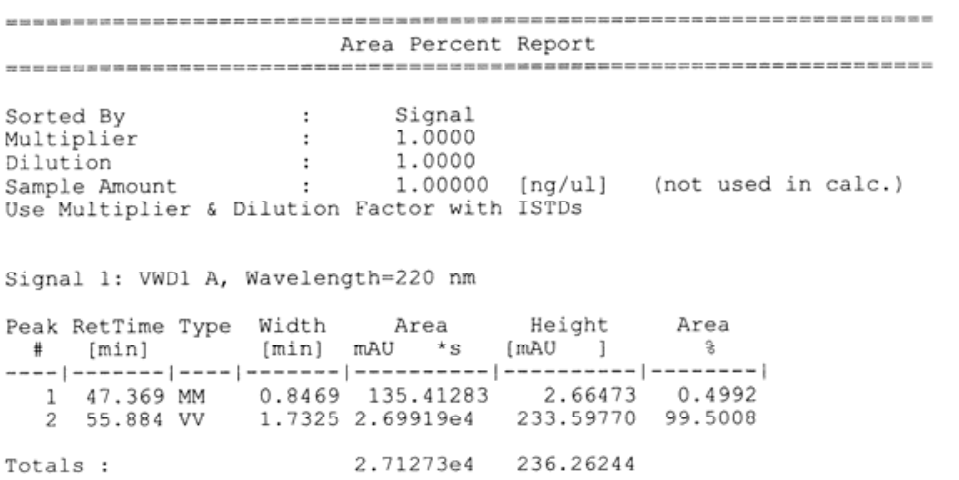




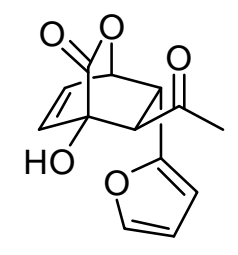

exo-7I
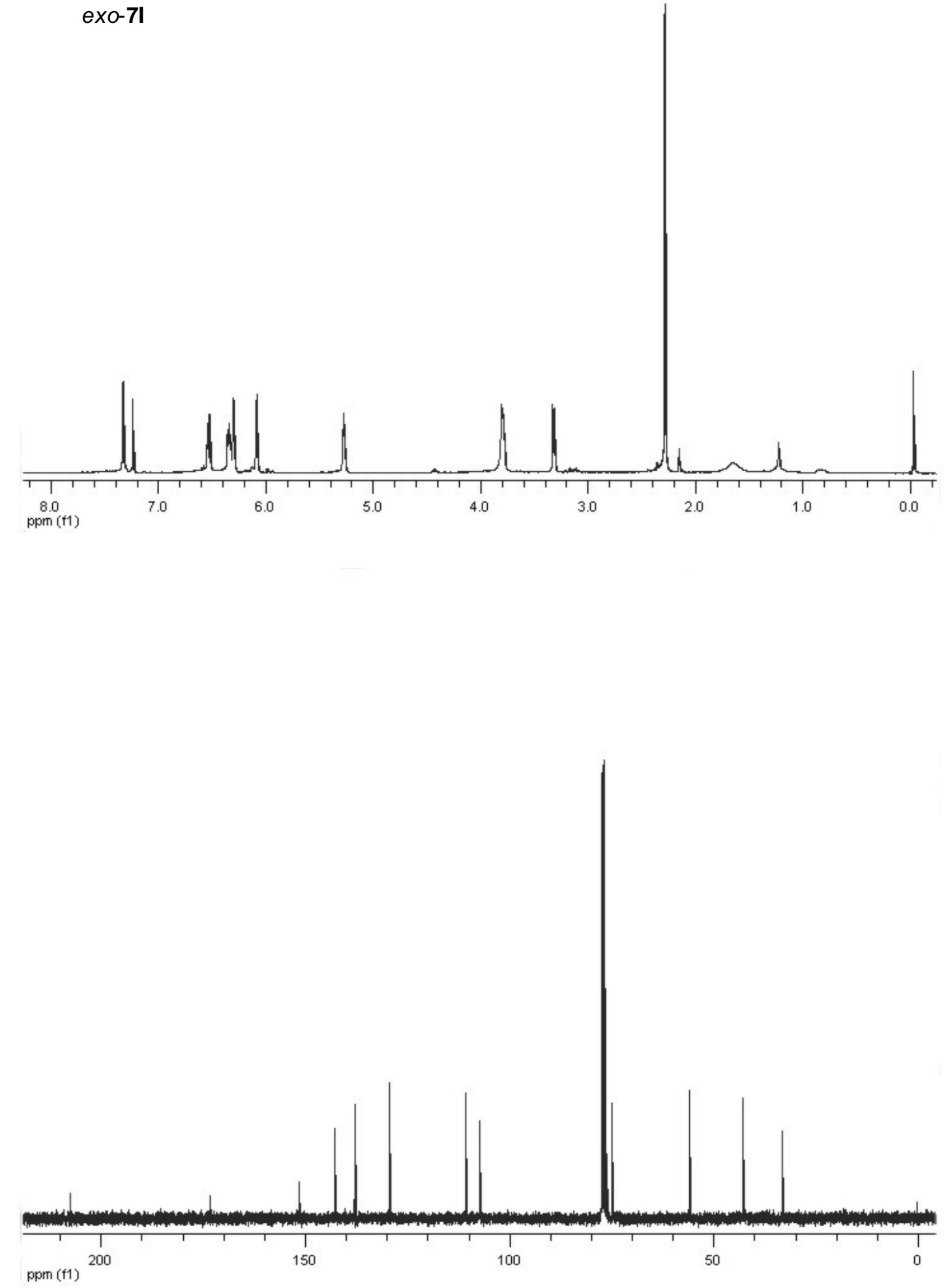

S 37 
HPLC, Chiralpak AS, hexanes: isopropanol $=95: 5,0.75 \mathrm{~mL} / \mathrm{min}, \lambda=220 \mathrm{~nm}$

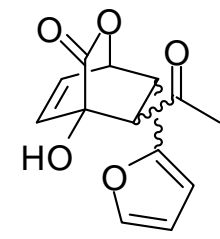

racemic-7I
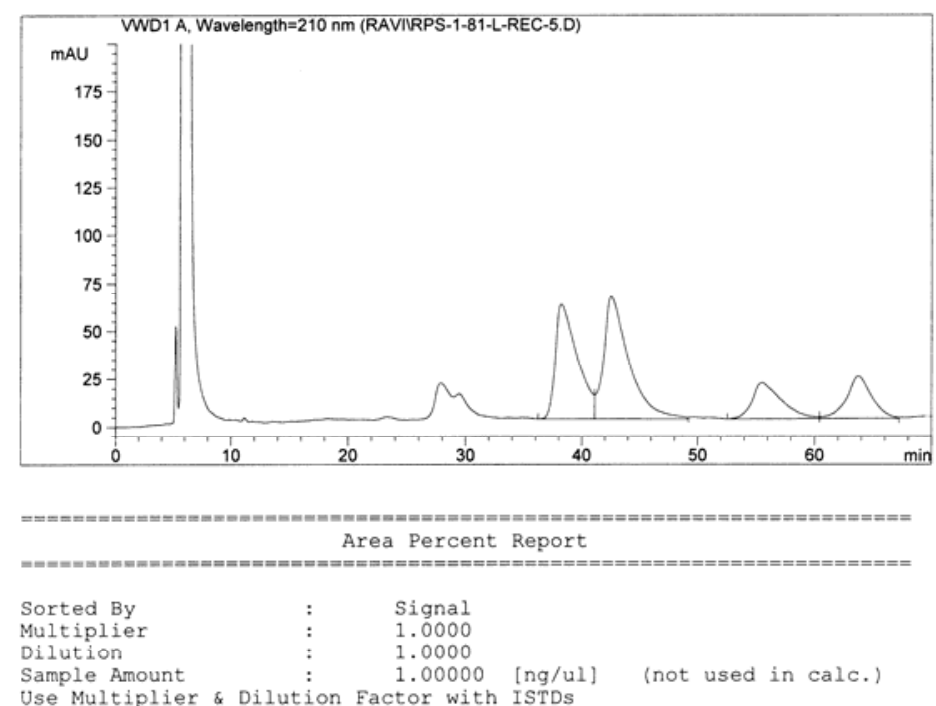

Signal 1: VWD1 $\mathrm{A}$, Wavelength=210 $\mathrm{nm}$

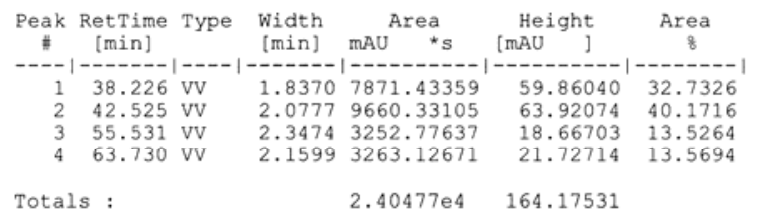

Totals :

$2.40477 \mathrm{e} 4 \quad 164.17531$
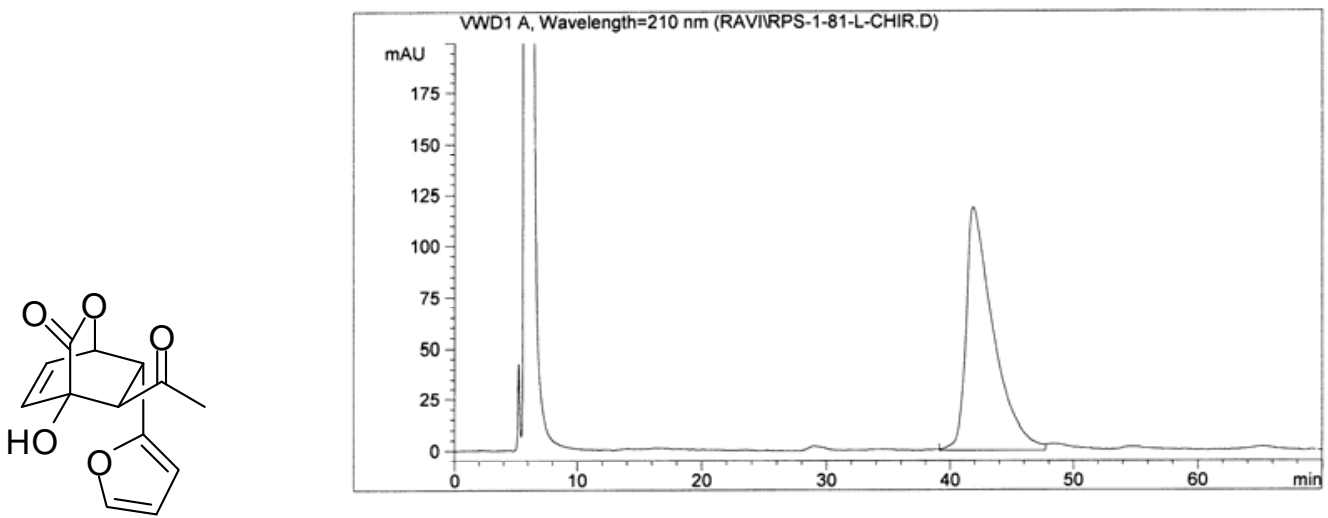

exo-7I, $99 \%$ ee obtained from QD-4 catalyzed reaction 

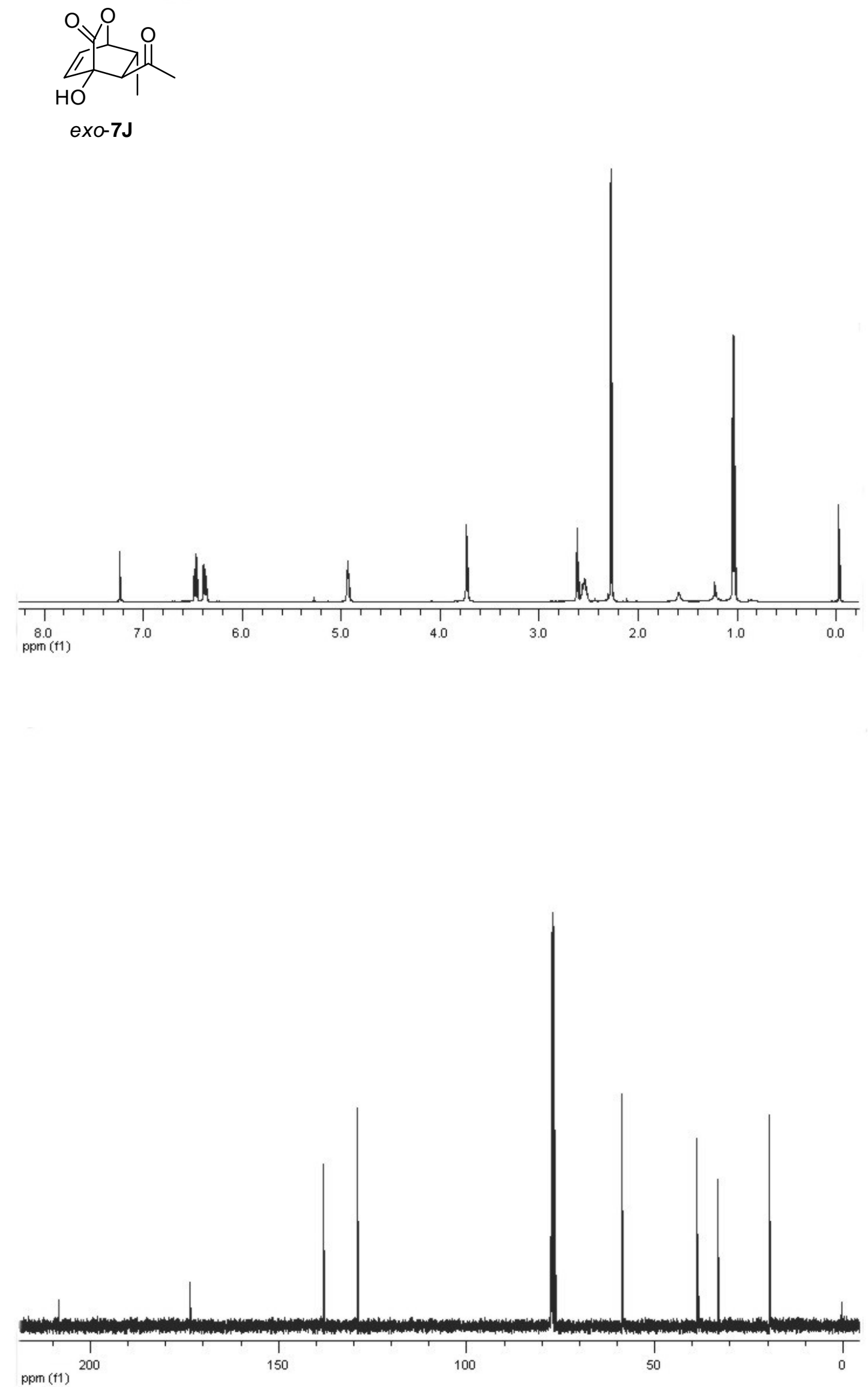

S 39 
HPLC, Chiralcel OD, hexanes: isopropanol $=95: 5,0.25 \mathrm{~mL} / \mathrm{min}, \lambda=215 \mathrm{~nm}$.

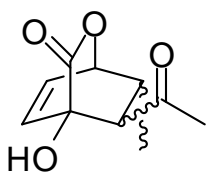

racemic-7J
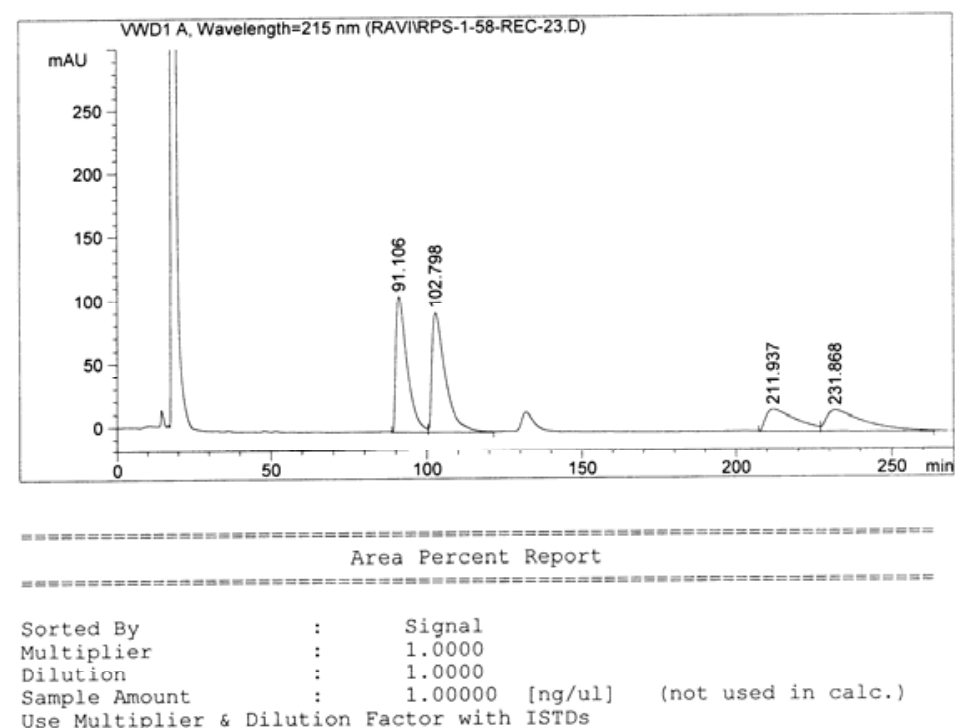

Signal 1: VWD1 A, Wavelength $=215 \mathrm{~nm}$

\begin{tabular}{|c|c|c|c|c|c|c|}
\hline Peak & RetTime & Type & Width & Area & Height & Area \\
\hline$\#$ & [min] & & {$[\mathrm{min}]$} & MAU & [mAU & 8 \\
\hline 1 & 91.106 & vV & 3.7209 & $2.69721 \mathrm{e} 4$ & 107.43987 & 33.2956 \\
\hline 2 & 102.798 & vv & 4.3200 & $2.80965 \mathrm{e} 4$ & 94.15569 & 34.6836 \\
\hline 3 & 211.937 & vV & 8.1958 & $1.17169 \mathrm{e} 4$ & 17.14172 & 14.4638 \\
\hline 4 & 231.868 & vV & 10.9830 & $1.42226 \mathrm{e} 4$ & 16.41351 & 17.5571 \\
\hline Tota & : & & & $8.10081 \mathrm{e} 4$ & 235.15079 & \\
\hline
\end{tabular}

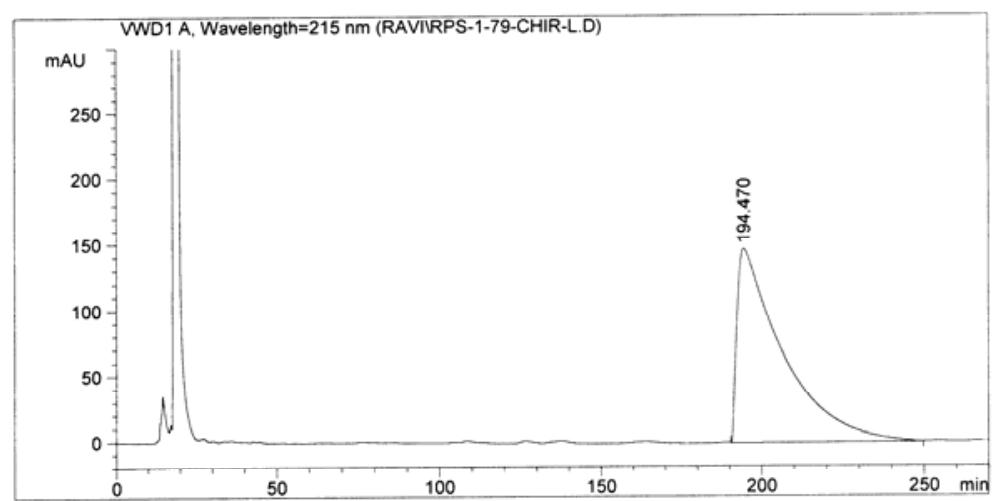

exo-7J, $99 \%$ ee obtained from QD-4 catalyzed reaction

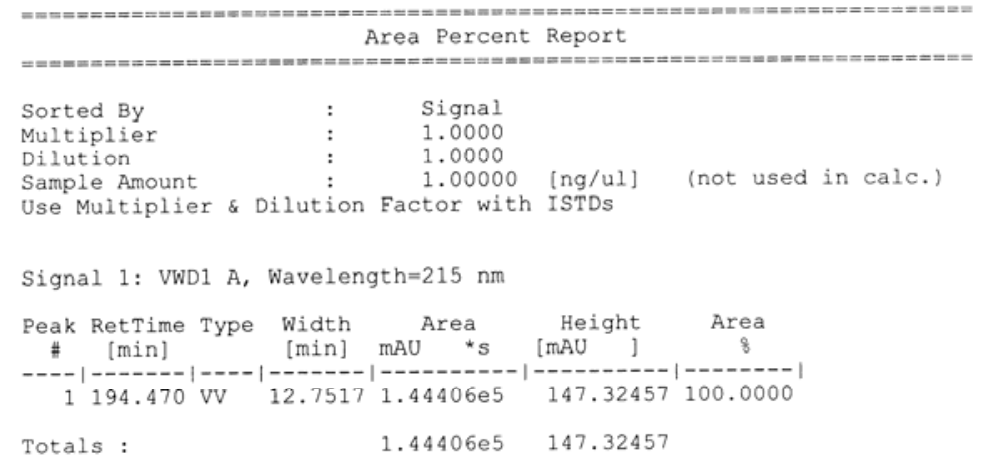




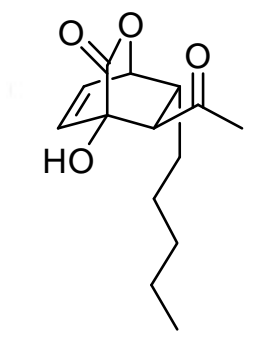

exo-7K

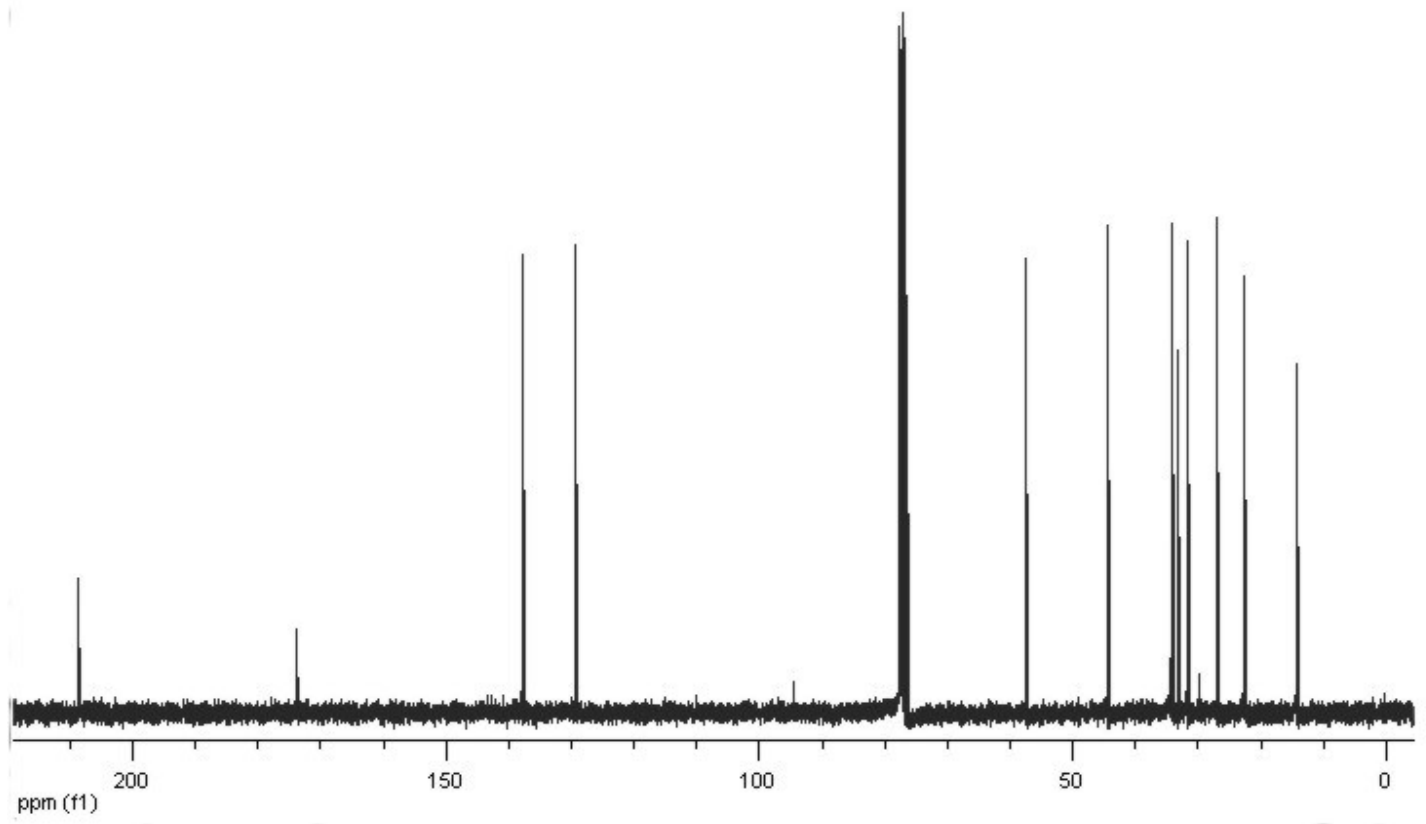

S 41 
HPLC, Chiralpak AD, hexanes: isopropanol $=97.5: 2.5,1.0 \mathrm{~mL} / \mathrm{min}, \lambda=215 \mathrm{~nm}$

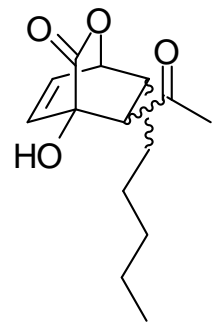

racemic-7K

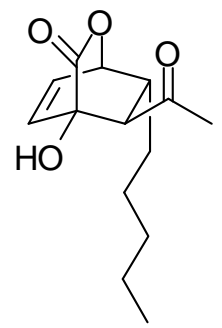

exo-7K, $99 \%$ ee obtained from QD-4 catalyzed reaction
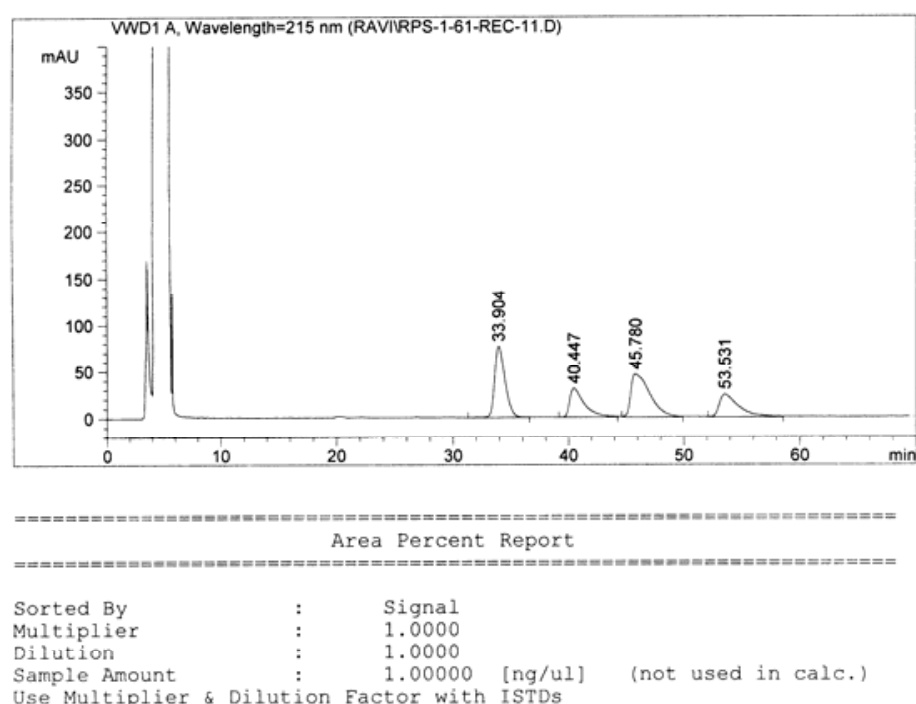

Use Multiplier \& Dilution Factor with ISTDS

Signal 1: VWD1 A, Wavelengt $h=215 \mathrm{~nm}$

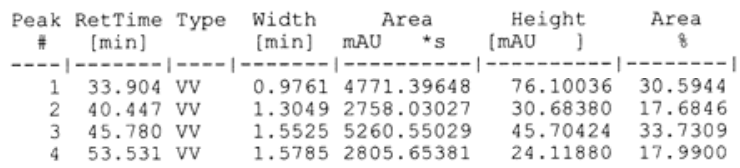

$\begin{array}{lll}\text { Totals : } & 1.55956 \mathrm{e} & 176.60719\end{array}$
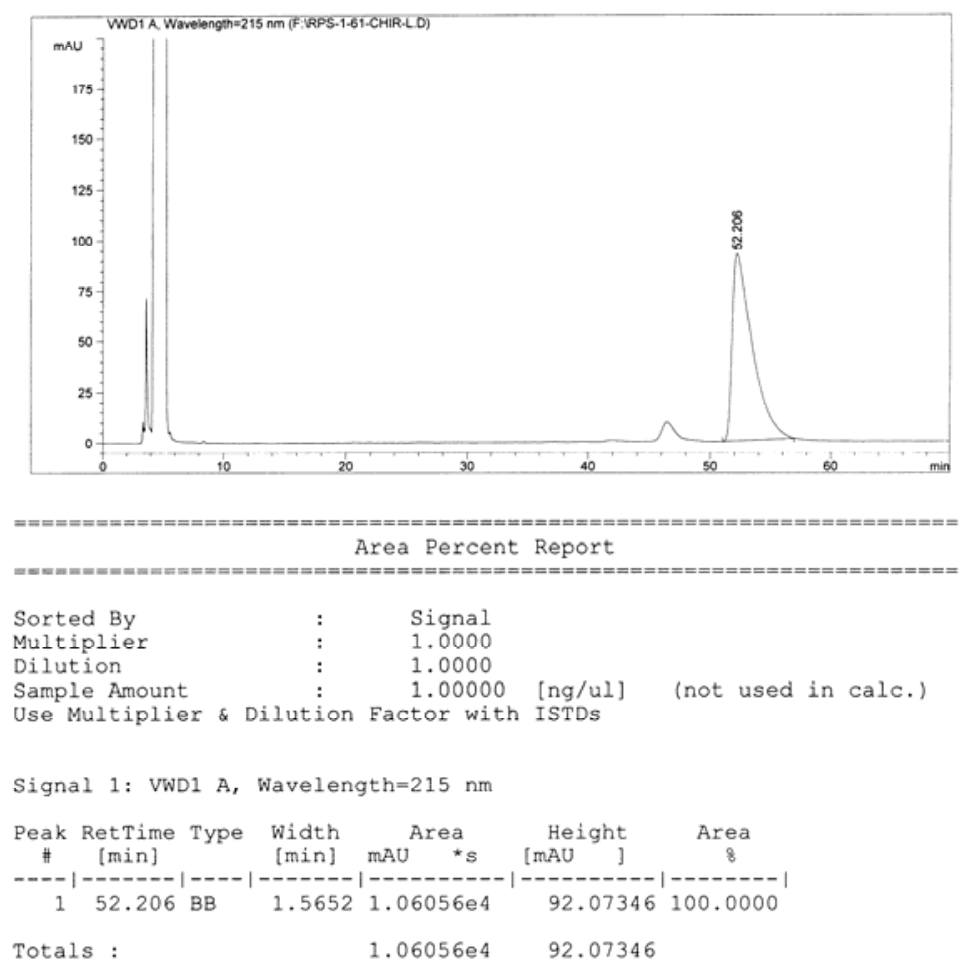


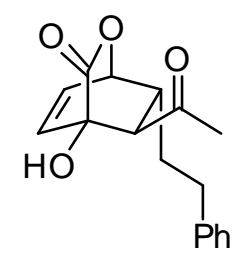

exo-7L

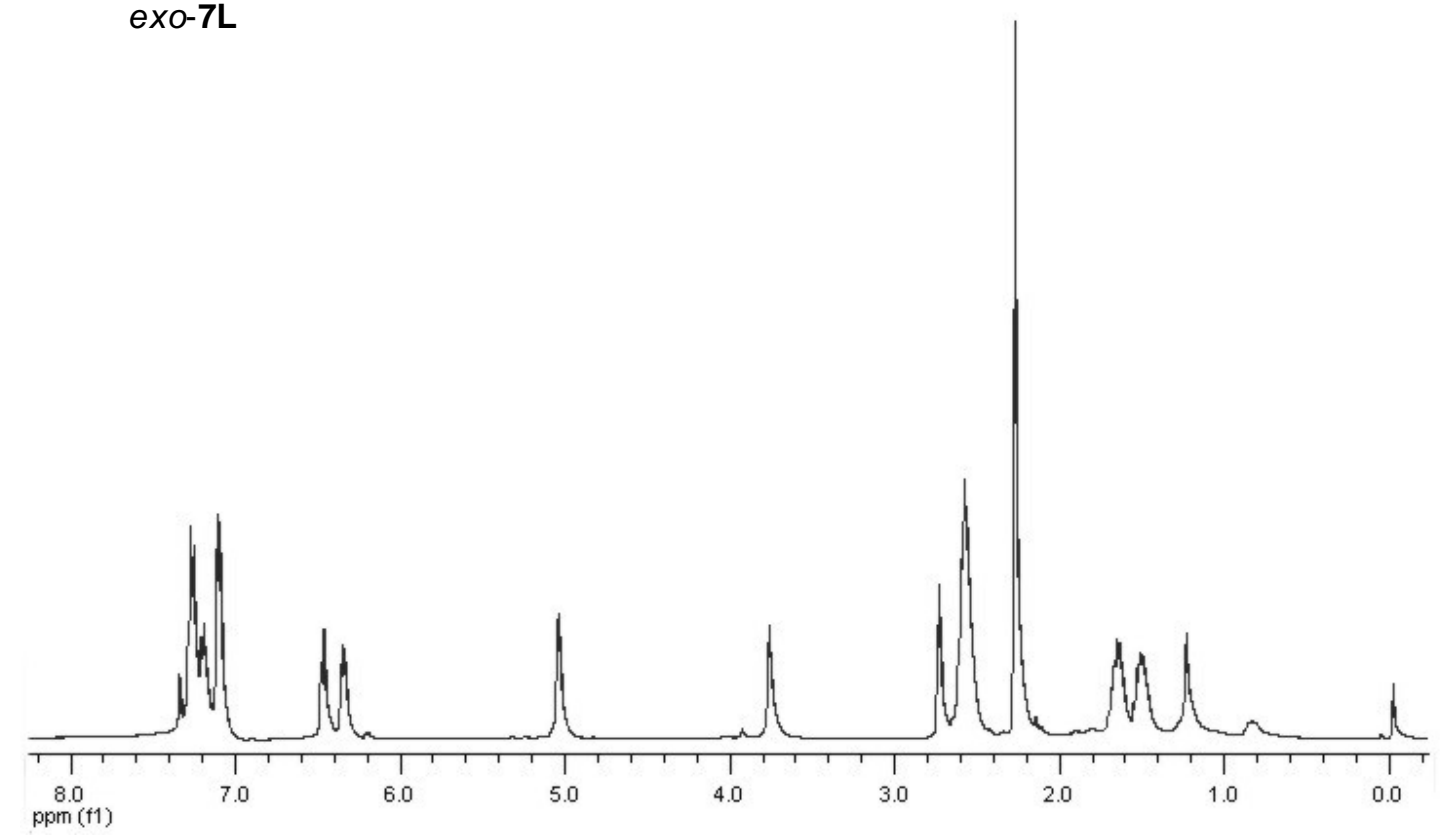

13C ósserve

Pu1se Sequence: s2pul

Solvent: CDC13

Ambient temperature
INOVA-400 $=f d^{*}$

Pulse 53.1 degrees

$1.199 \mathrm{Eec}$

wiath $25000.0 \mathrm{gz}$

OBSRRVE C13, 100.5180102 $\mathrm{MH}$

DECOUPLE H1, 399.7552472 MHz

power 37 ab

on during acquinition

off during dolay

DATA PRoCESSTNG

Line broadening 1.0 k

Fr size 65536
Total time $1 \mathrm{hr}, 20 \mathrm{~min}, 32$ sec

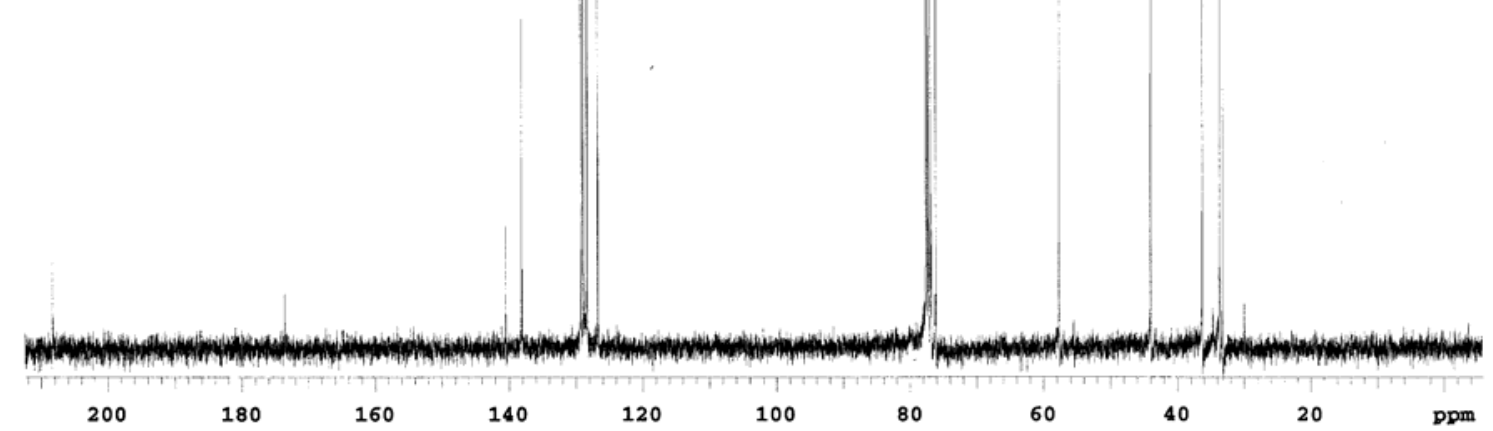


HPLC, Chiralpak AD, hexanes: isopropanol $=60: 40,0.5 \mathrm{~mL} / \mathrm{min}, \lambda=220 \mathrm{~nm}$

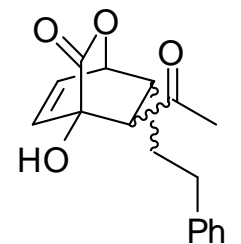

racemic- $\mathbf{7 L}$
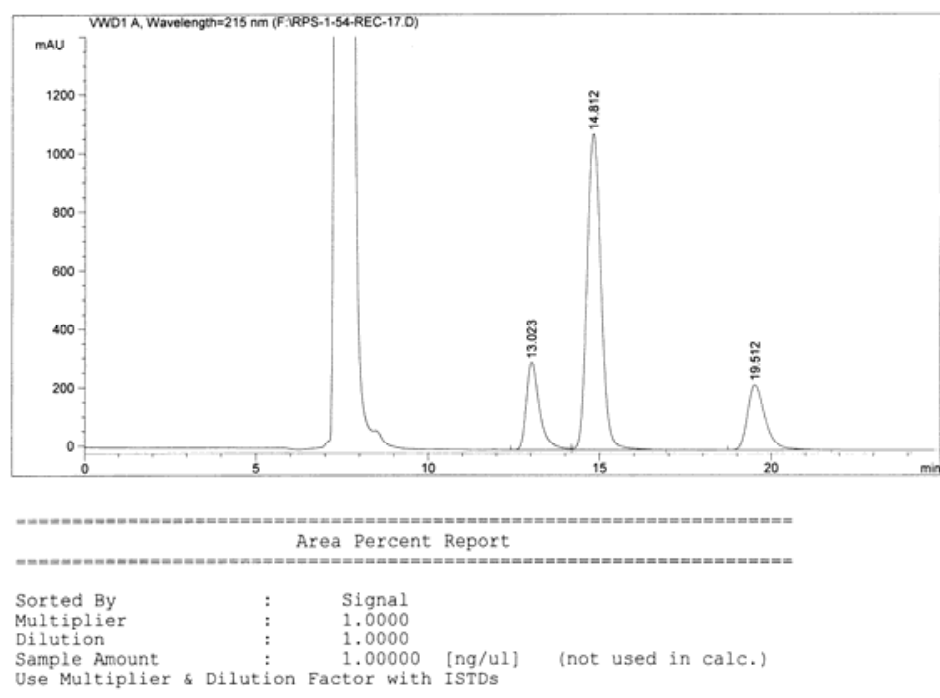

Factor with ISTDs

Signal 1: VWD1 $A$, Wavelength $=215 \mathrm{~nm}$

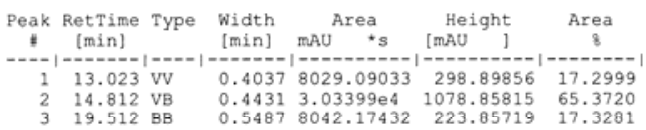

Totals : $\quad 4.64112 \mathrm{e} 4 \quad 1601.61391$
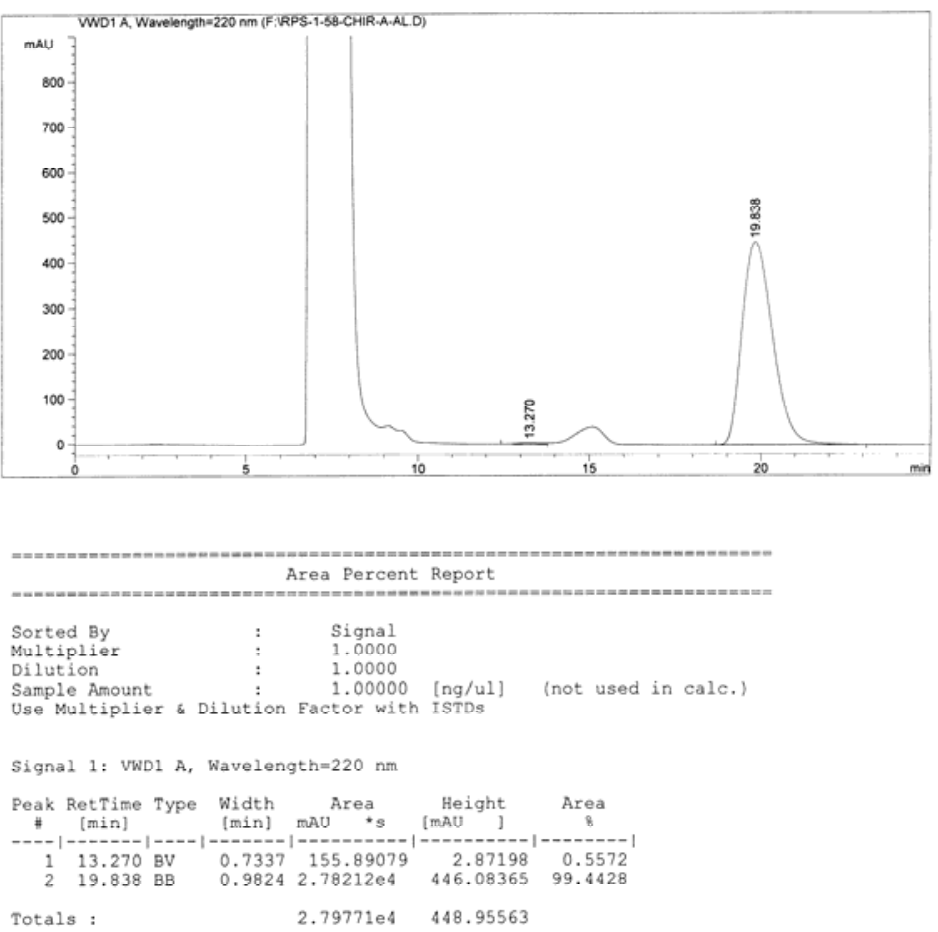

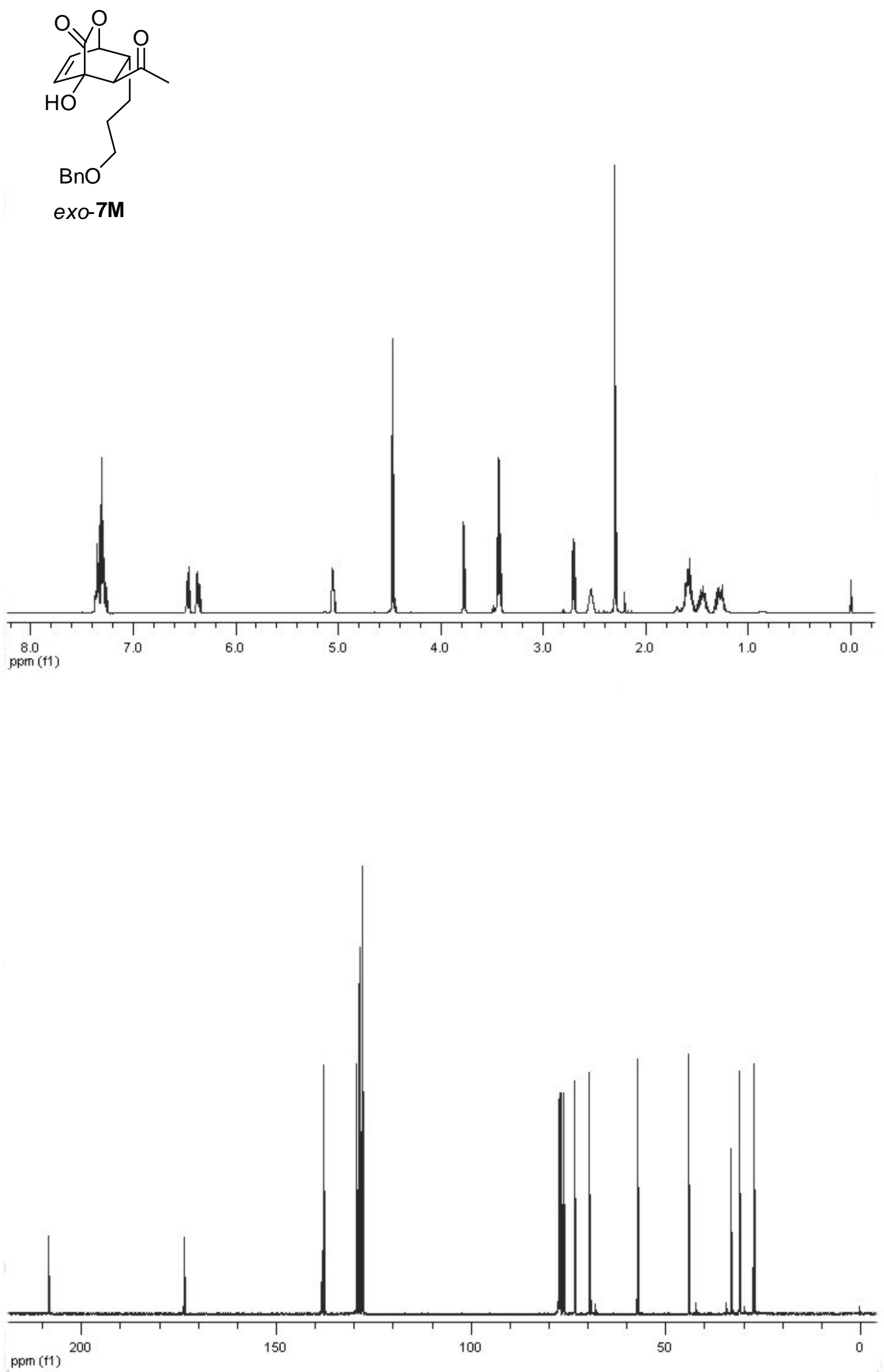
HPLC, Chiralcel OD, hexanes: isopropanol $=90: 10,1.0 \mathrm{~mL} / \mathrm{min}, \lambda=220 \mathrm{~nm}$.

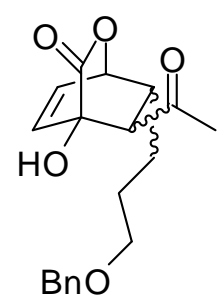

racemic- $\mathbf{7 M}$
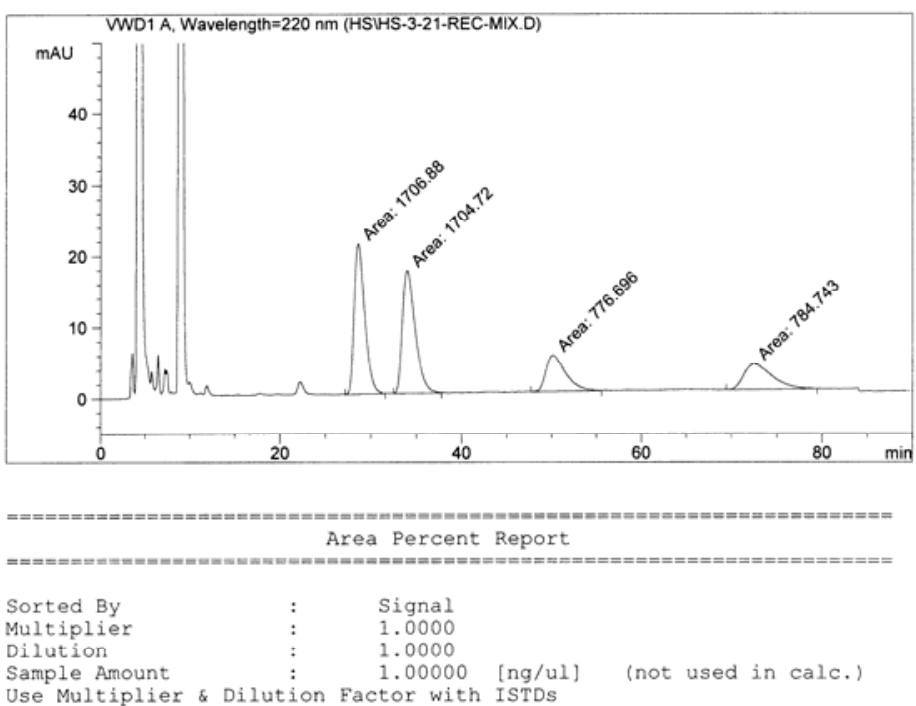

Use Multiplier \& Dilution Factor with ISTDs

Signal 1: VWD1 $A$, Wavelengt $h=220 \mathrm{~nm}$

\begin{tabular}{|c|c|c|c|c|c|c|}
\hline eak & $\begin{array}{l}\text { RetTime } \\
\text { [min] }\end{array}$ & Type & $\begin{array}{l}\text { Width } \\
\text { [min] }\end{array}$ & mAU ${ }^{\text {Area }}{ }^{*} \mathrm{~s}$ & $\begin{array}{c}\text { Height } \\
{[\mathrm{mAU}]}\end{array}$ & $\begin{array}{c}\text { Area } \\
8\end{array}$ \\
\hline $\begin{array}{l}1 \\
2 \\
3 \\
4\end{array}$ & $\begin{array}{l}28.591 \\
33.981 \\
50.165 \\
72.442\end{array}$ & $\begin{array}{l}\text { MM } \\
\text { MM } \\
M M \\
M M\end{array}$ & $\begin{array}{l}1.3489 \\
1.6480 \\
2.5440 \\
3.6038\end{array}$ & $\begin{array}{r}1706.87891 \\
1704.71936 \\
776.69598 \\
784.74298\end{array}$ & $\begin{array}{r}21.08998 \\
17.24040 \\
5.08840 \\
3.62920\end{array}$ & $\begin{array}{l}34.3227 \\
34.2792 \\
15.6181 \\
15.7800\end{array}$ \\
\hline $\mathrm{a}$ & & & & 973.0372 & 47.0 & \\
\hline
\end{tabular}
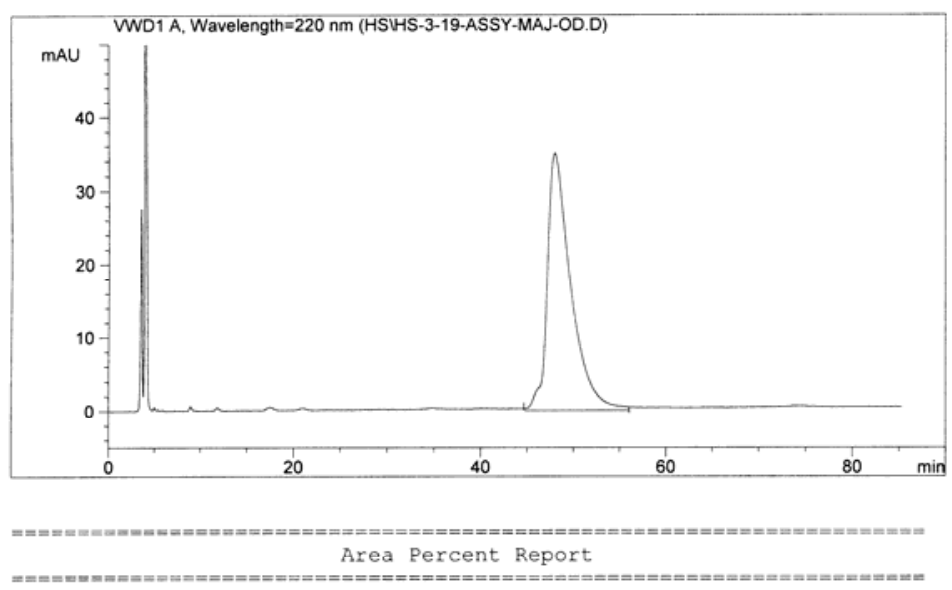

Sorted By : Signal Multiplier : 1.0000 Dilution : $\quad 1.0000$

1.00000 [ng/ul] (not used in calc.)

Use Multiplier \& Dilution Factor with ISTDs

Signal 1: VWD1 $A$, wavelength $=220 \mathrm{~nm}$

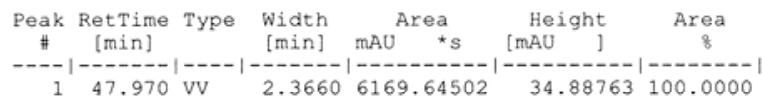

Totals : $\quad 6169.64502 \quad 34.88763$ 

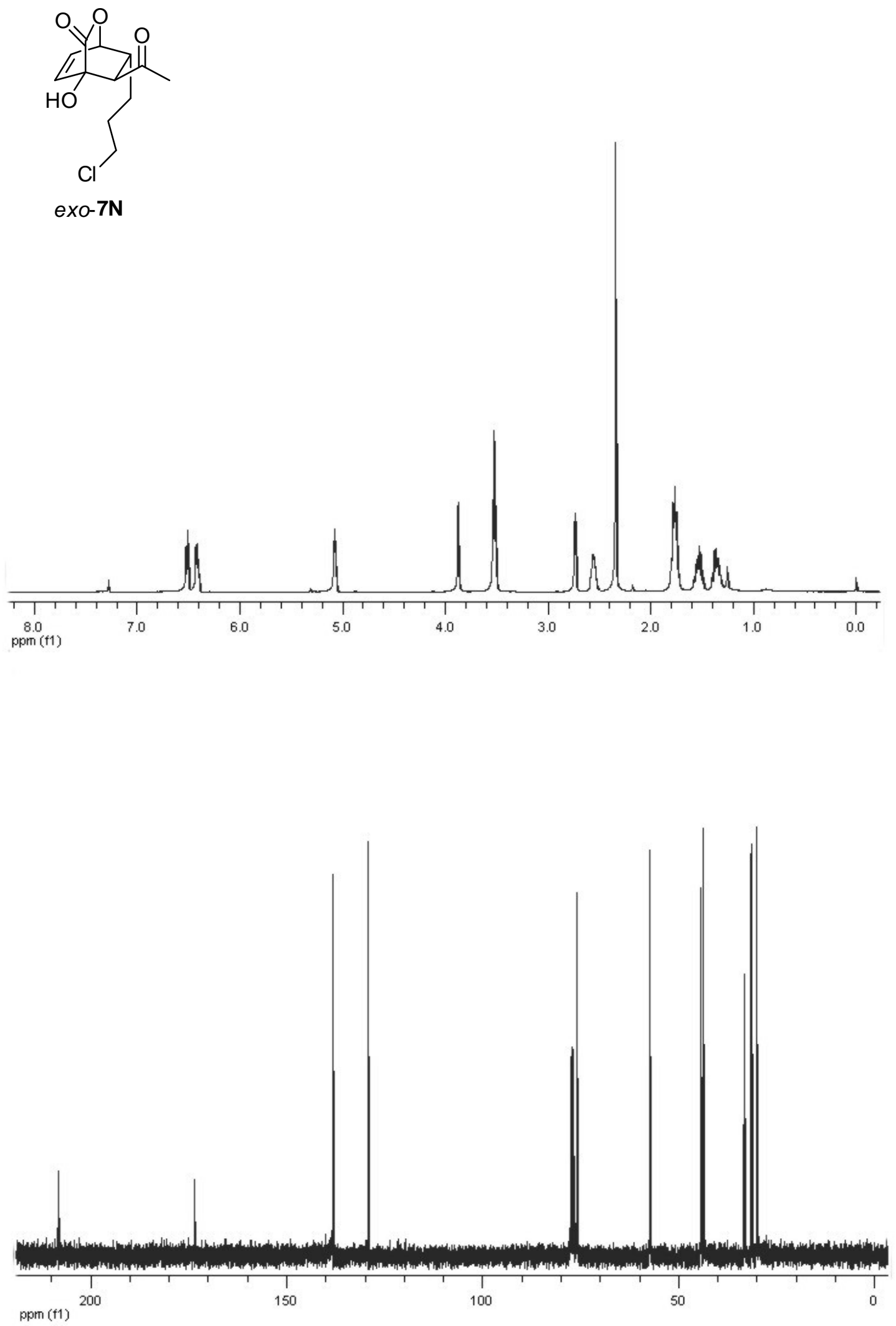
HPLC, Chiralpak AD $+(R, R)$-Whelk-O 1, hexanes: isopropanol $=75: 25,1.0 \mathrm{~mL} / \mathrm{min}$, $220 \mathrm{~nm}$
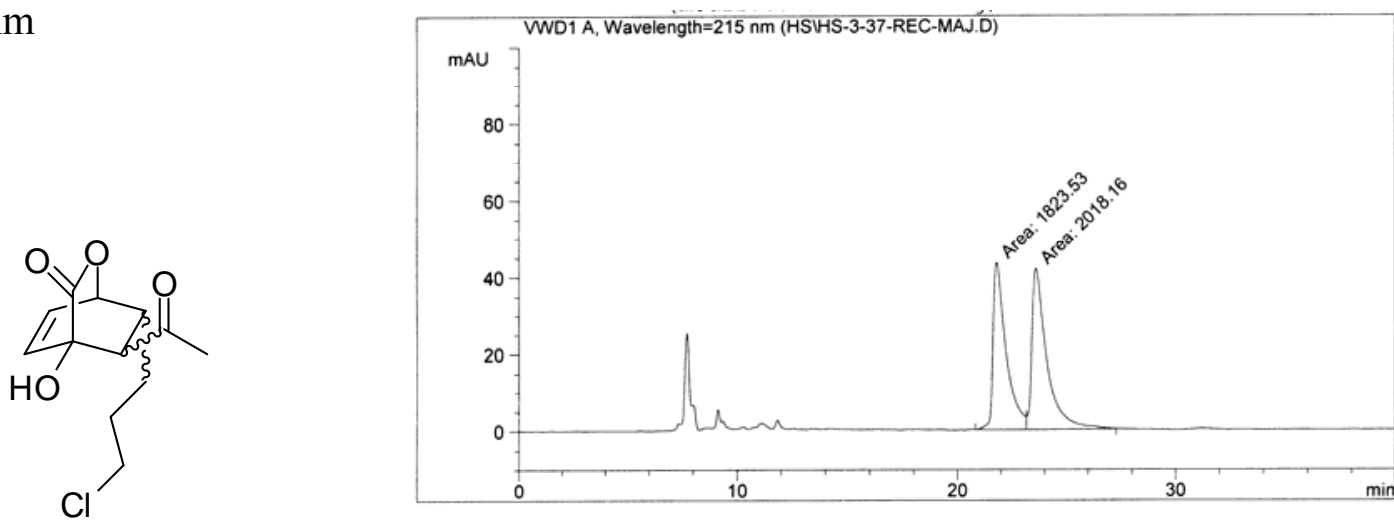

racemic- $\mathbf{7 N}$

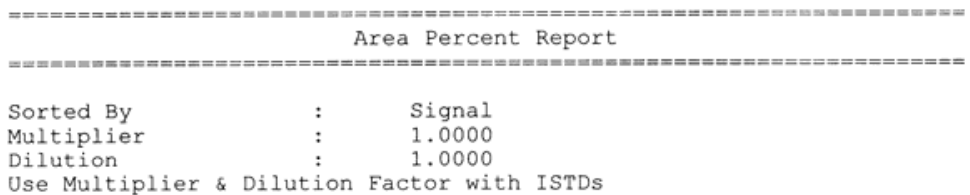

Use Multiplier \& Dilution Factor with ISTDs

Signal 1: VWD1 A, Wavelength=215 nm

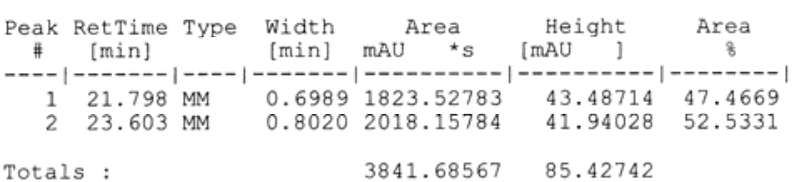

Totals :

$3841.68567 \quad 85.42742$

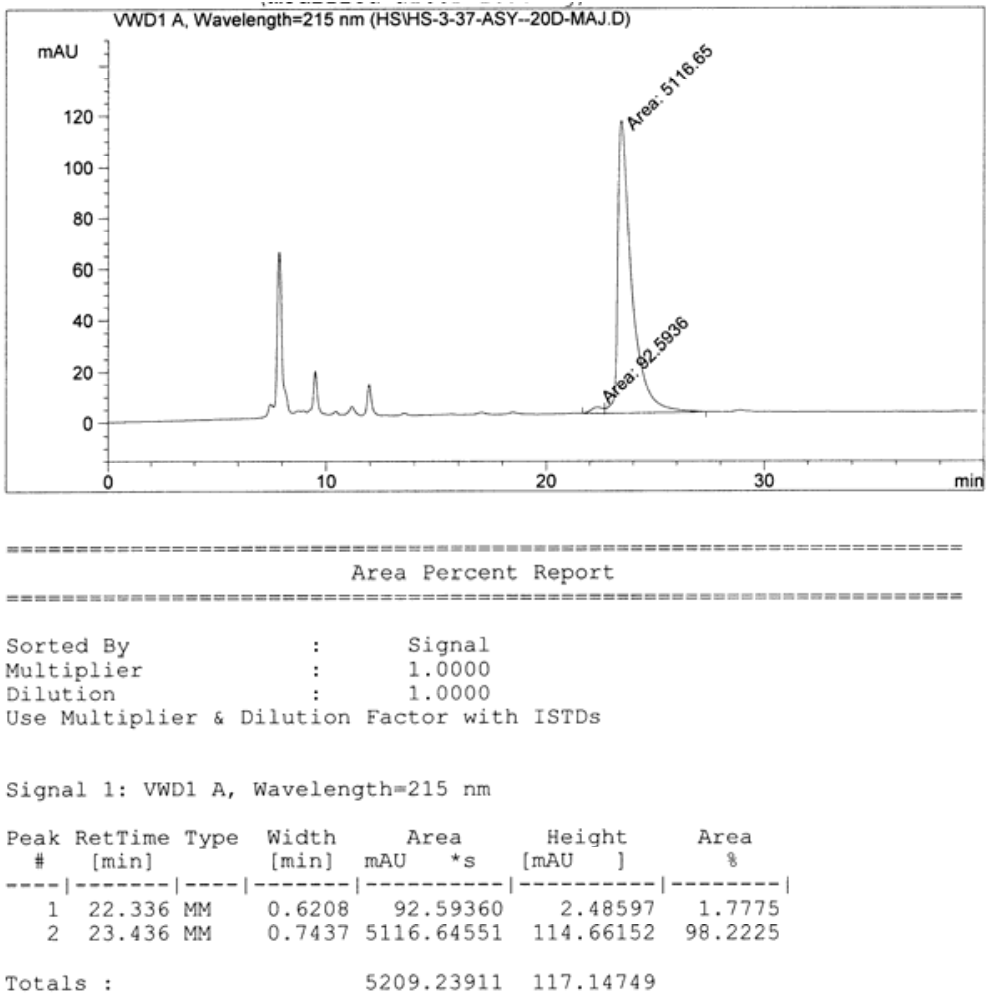




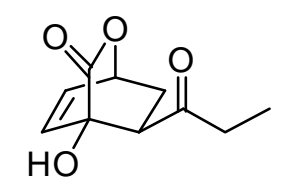

exo-70
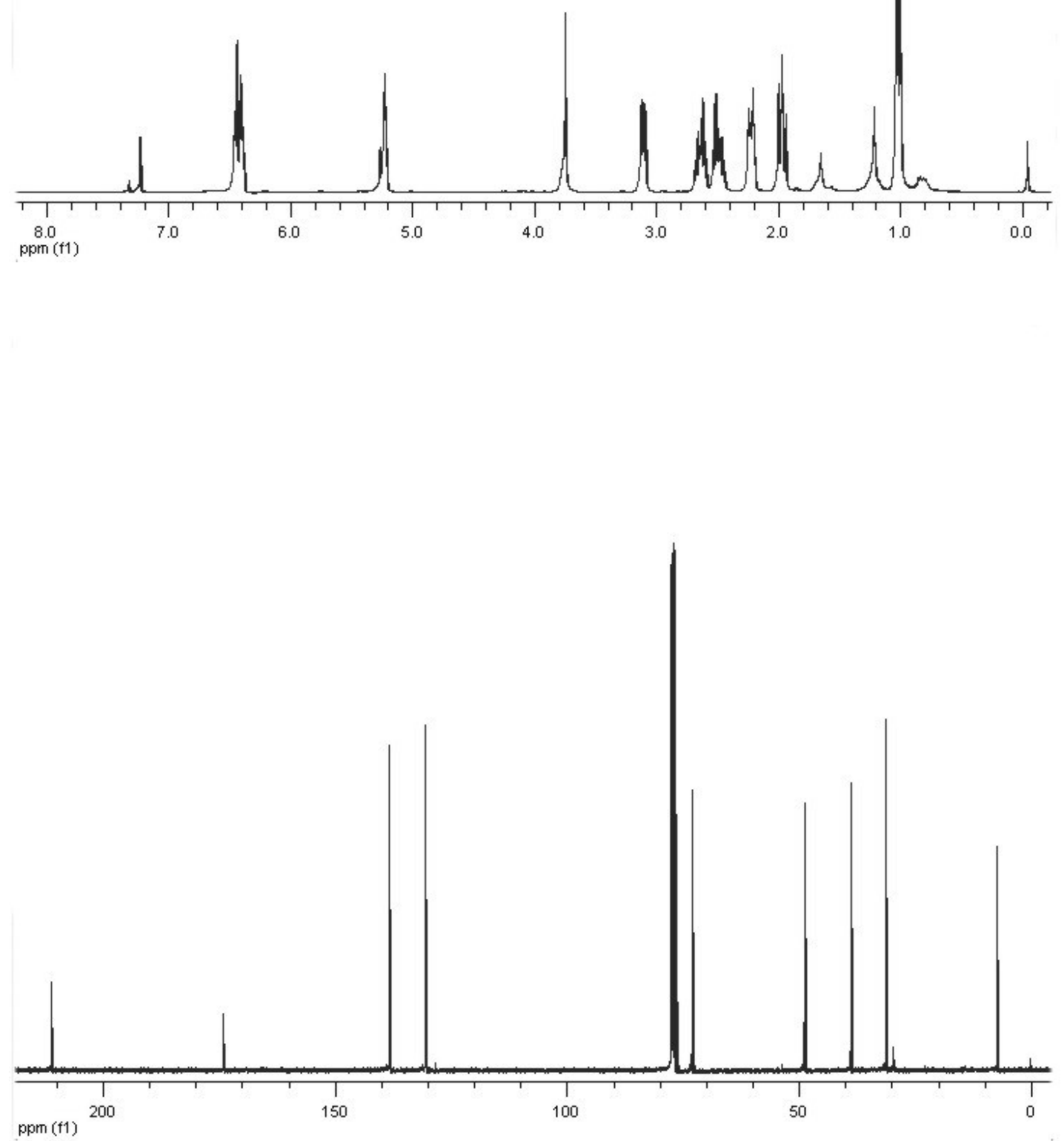
HPLC, Chiralpak AD, hexanes: isopropanol $=85: 15,1.0 \mathrm{~mL} / \mathrm{min}, \lambda=220 \mathrm{~nm}$
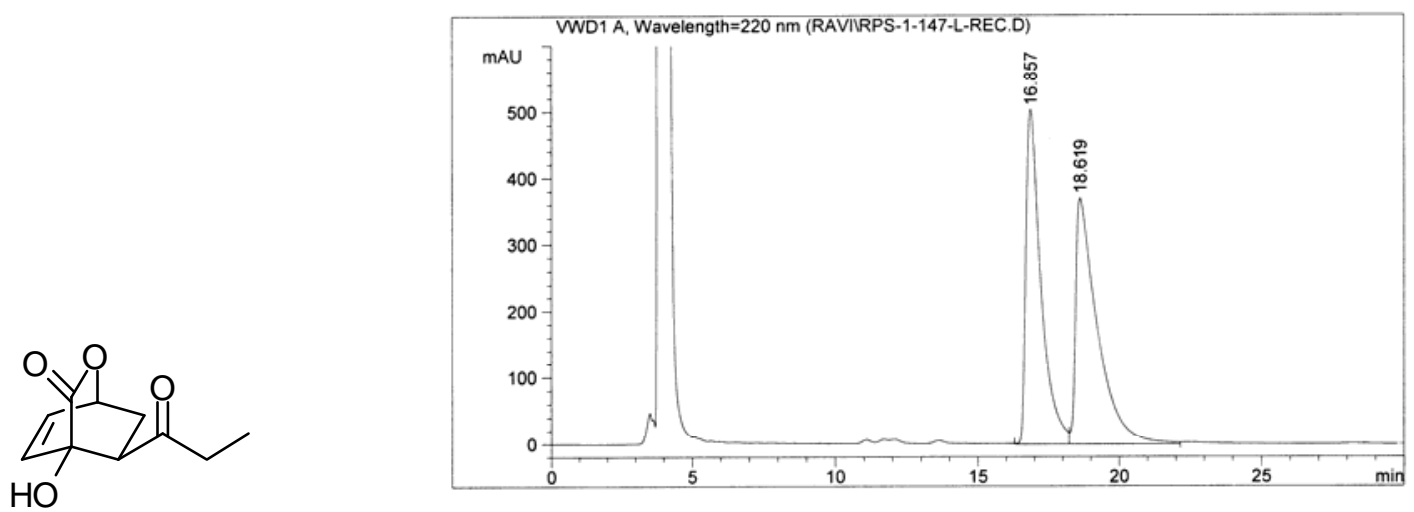

racemic-exo-70

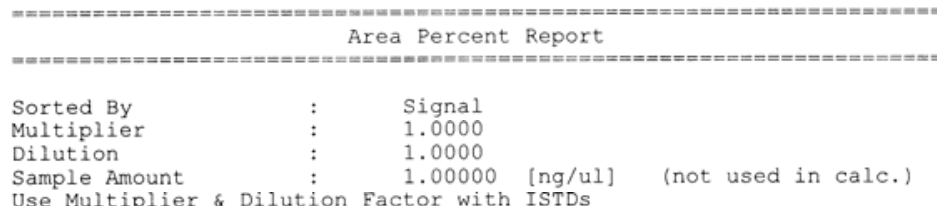

or with ISTDS

Signal 1: VWD1 A, Wavelengt $h=220 \mathrm{~nm}$

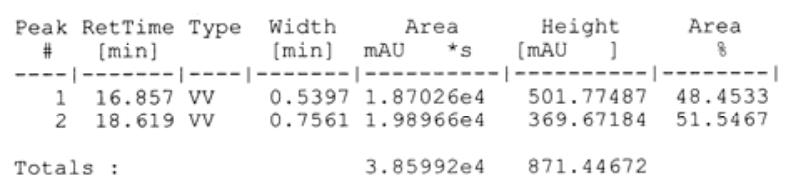

Totals :

$3.85992 \mathrm{e} 4 \quad 871.44672$

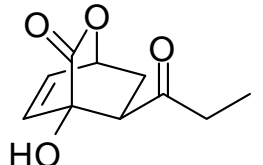

exo-70, $99 \%$ ee obtained from QD-4 catalyzed reaction
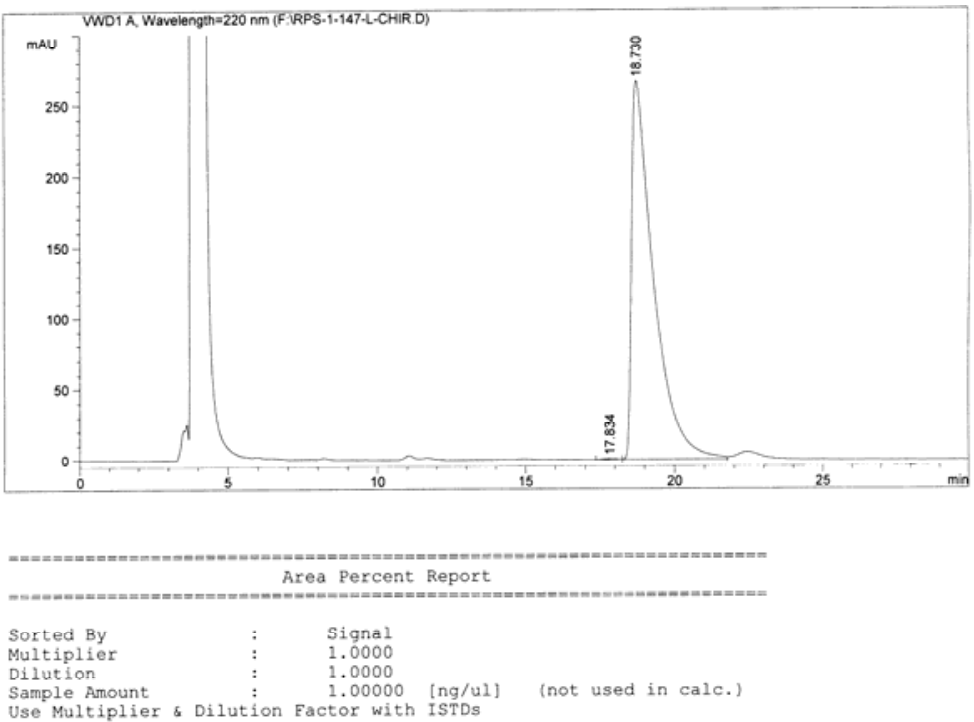

Sample Amount
Use Multiplier \& Dilution Factor with 1 ISTDs

Signal 1: VWD1 $\mathrm{A}$, wavelength $=220 \mathrm{~nm}$

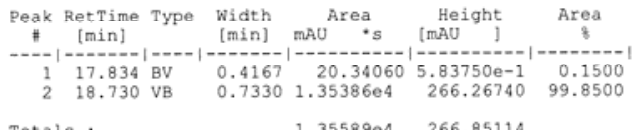

Totals :

$1.35589 \mathrm{e} 4 \quad 266.85114$ 

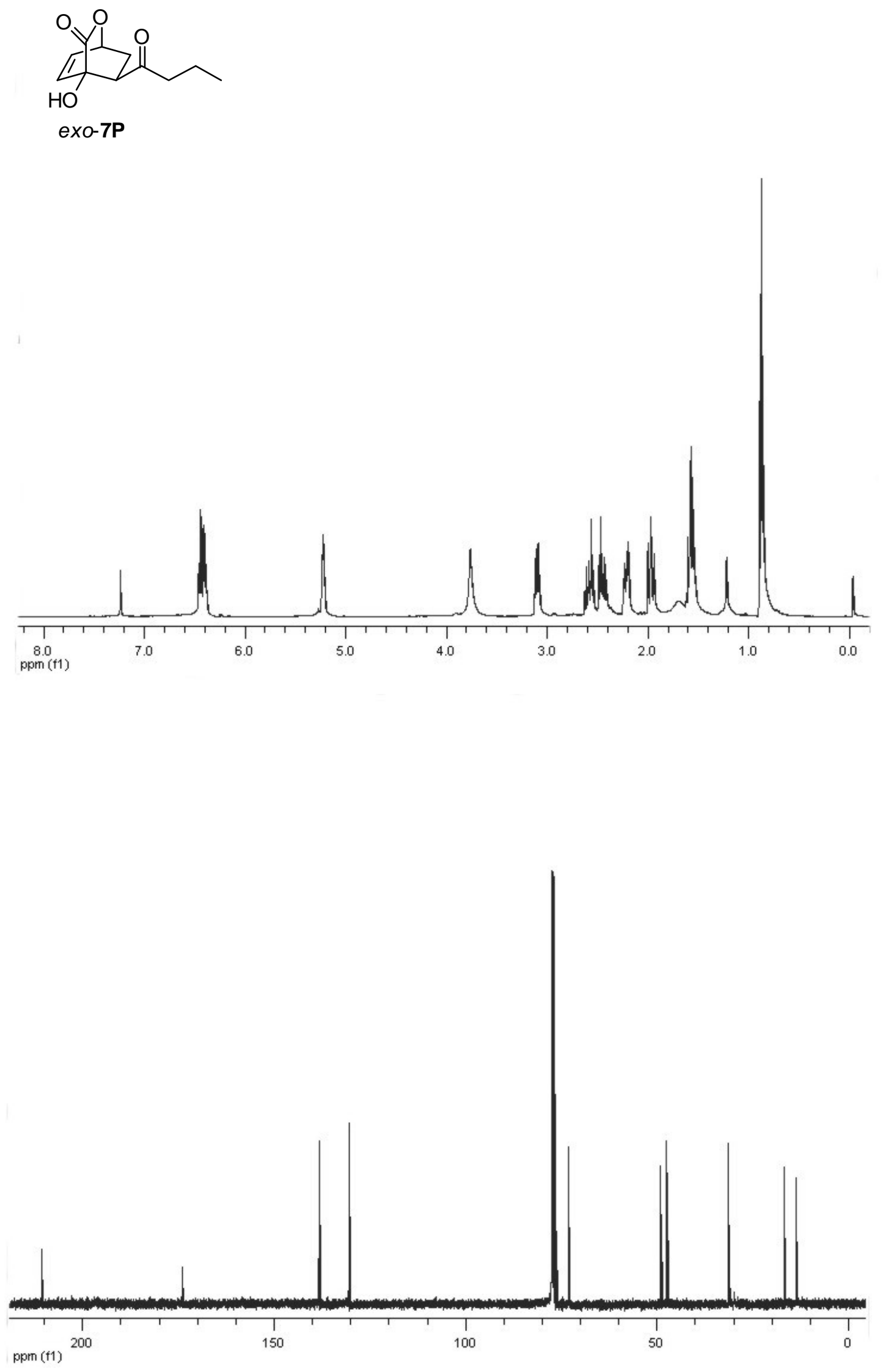

S 51 
HPLC, Chiralpak AD, hexanes: isopropanol $=90: 10,1.0 \mathrm{~mL} / \mathrm{min}, \lambda=220 \mathrm{~nm}$

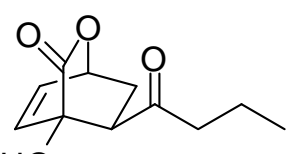

$\mathrm{HO}$

racemic-exo-7P

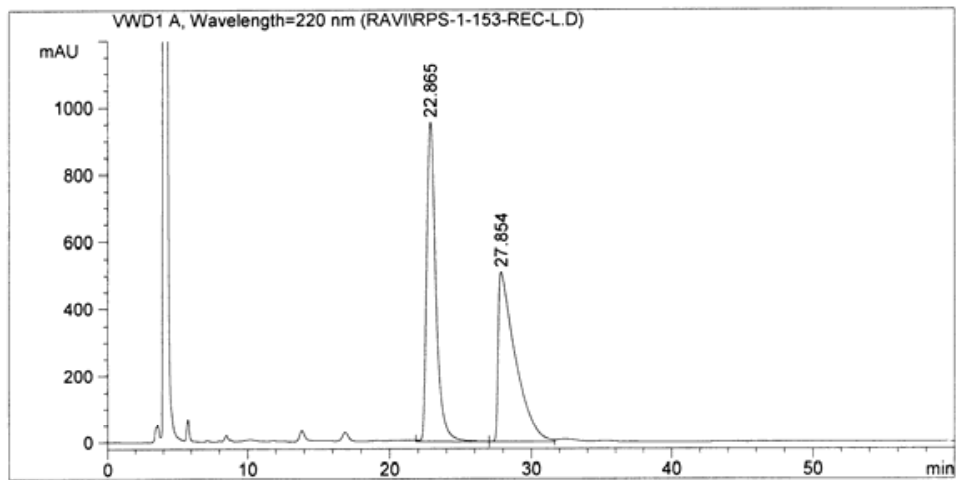

Area Percent Report

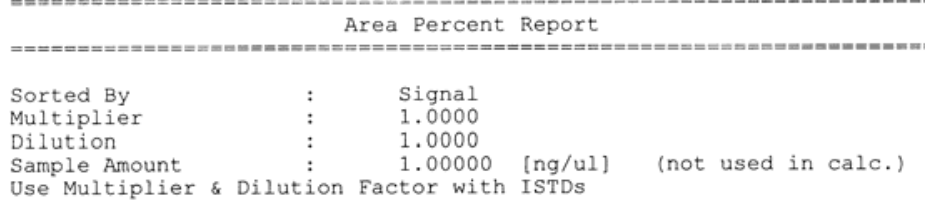

Signal 1: VWD1 $\mathrm{A}$, Wavelengt $\mathrm{h}=220 \mathrm{~nm}$
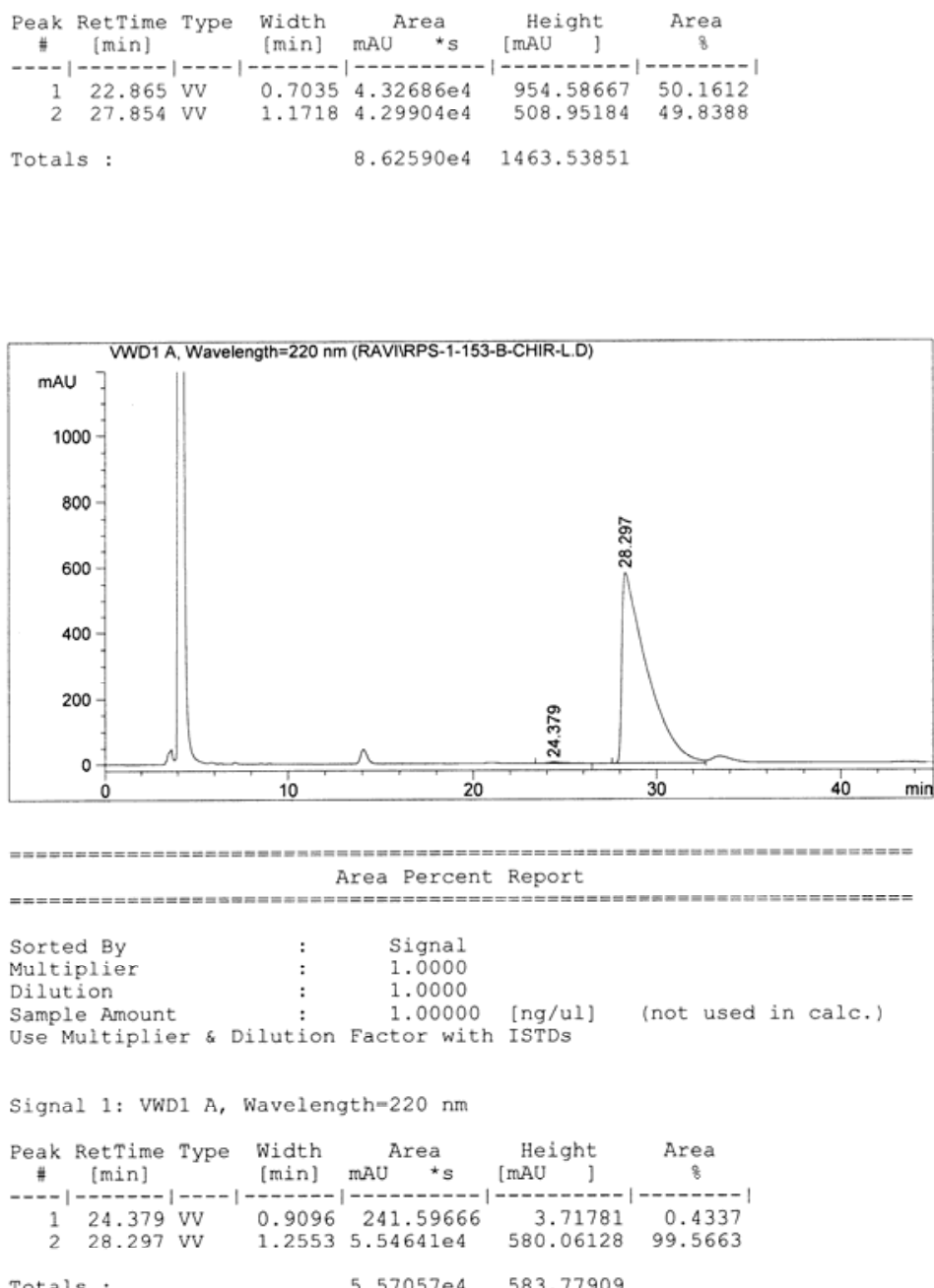

Totals :

5.57057 e4 583.77909 

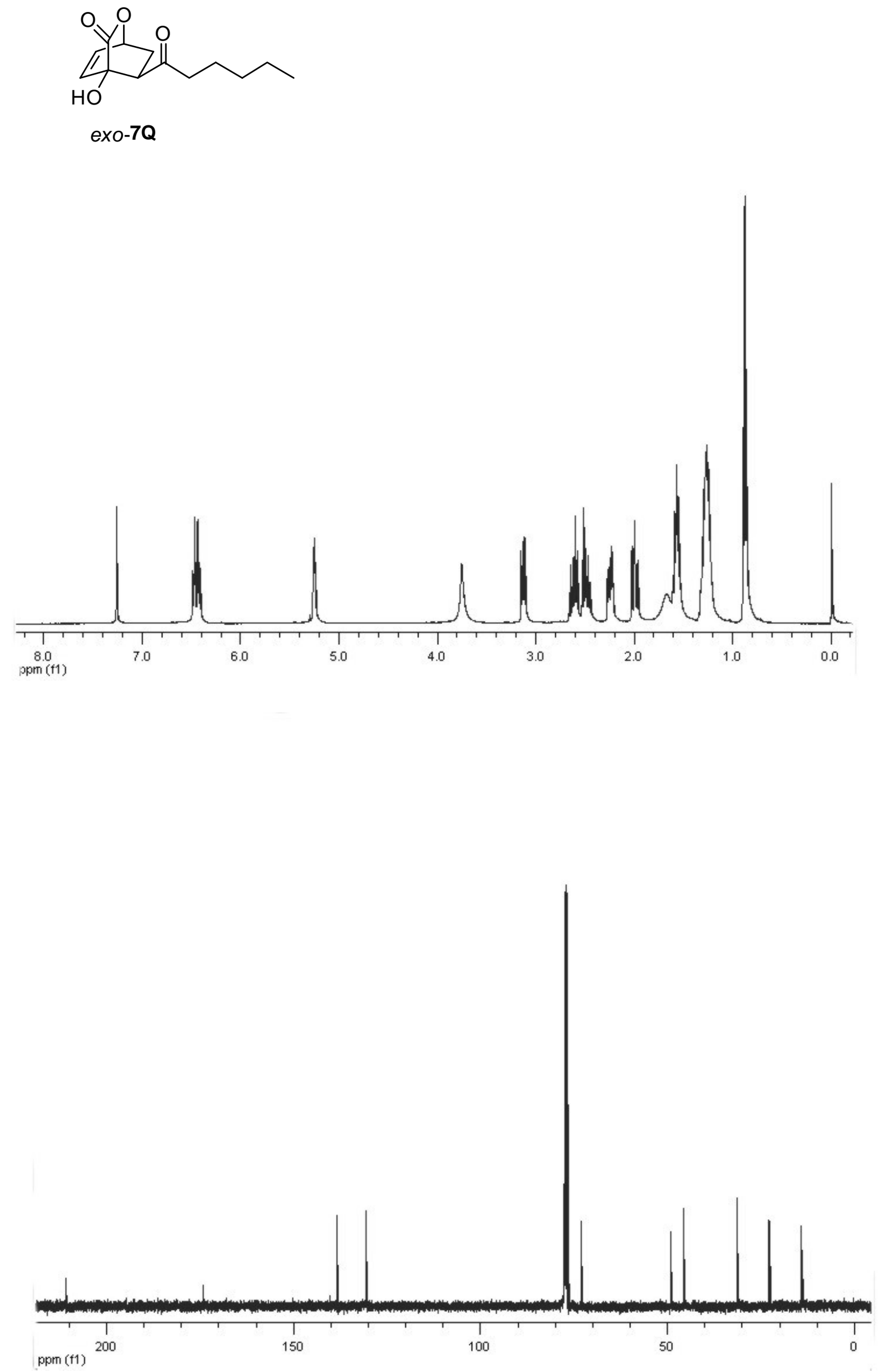

S 53 
HPLC, Chiralpak AD, hexanes: isopropanol $=90: 10,1.0 \mathrm{~mL} / \mathrm{min}, \lambda=220 \mathrm{~nm}$

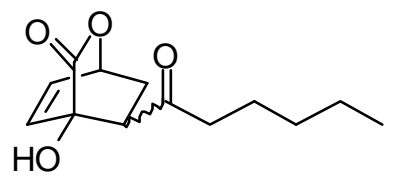

racemic-7Q
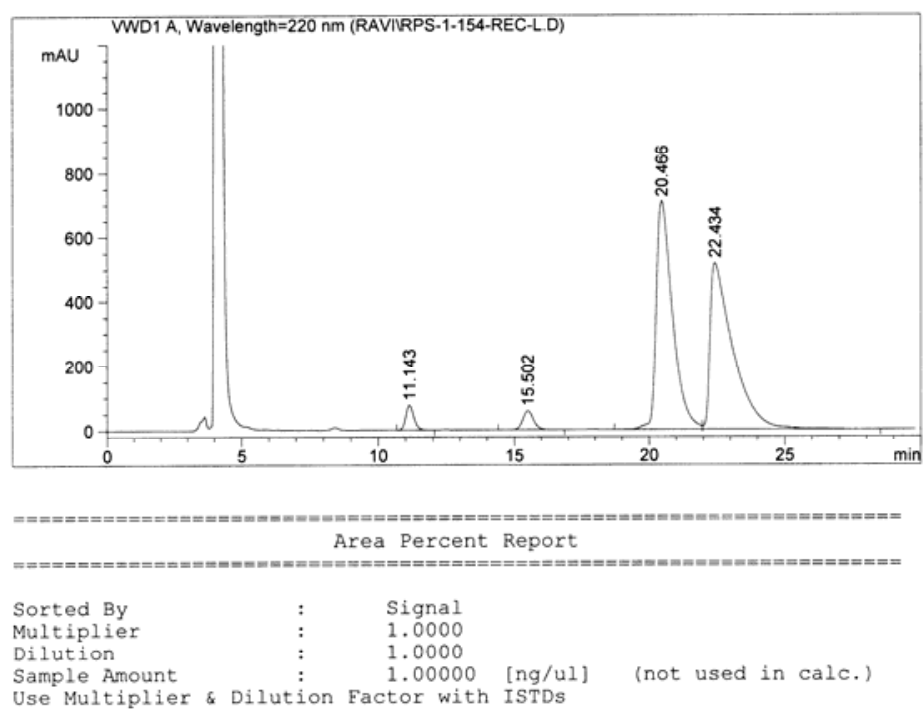

Signal 1: VWD1 A, Wavelength=220 nm

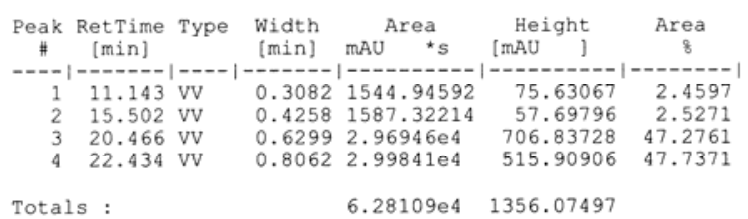

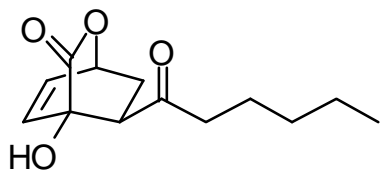

exo-7Q, $96 \%$ ee obtained from QD-4 catalyzed reaction
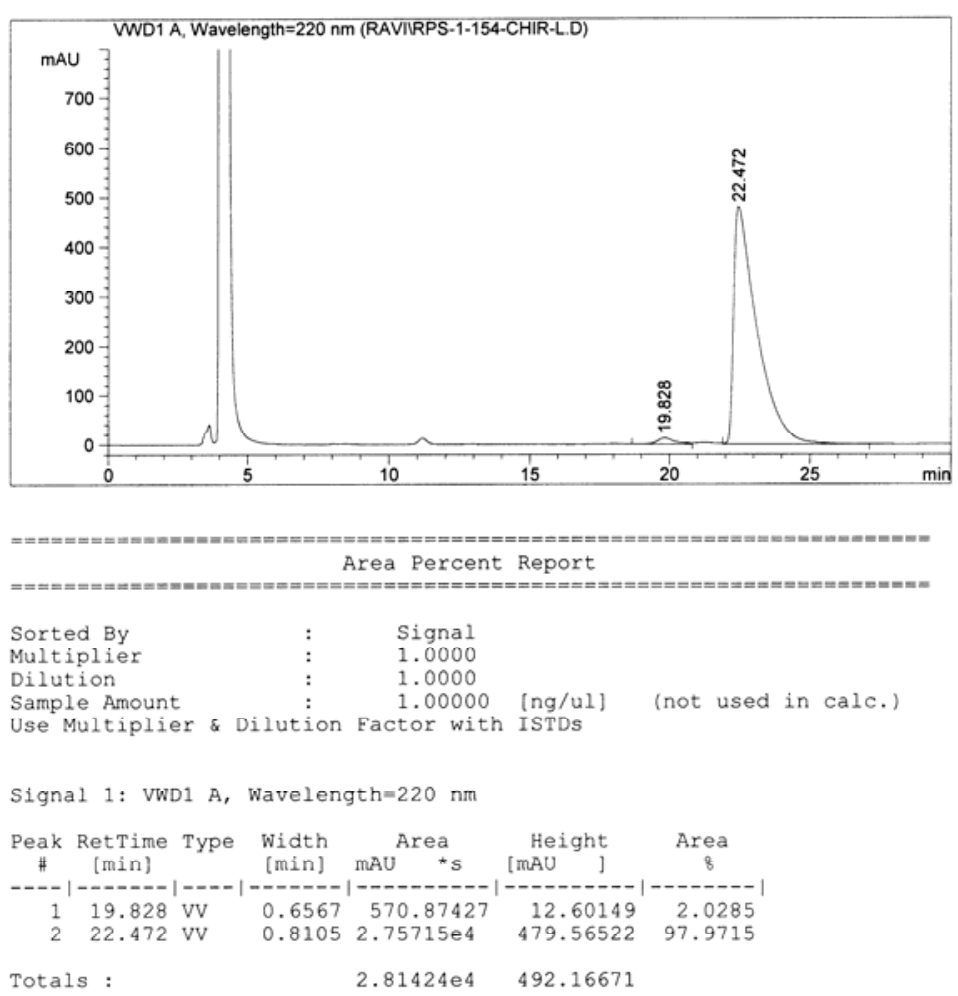


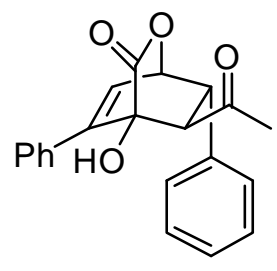

exo-7A'
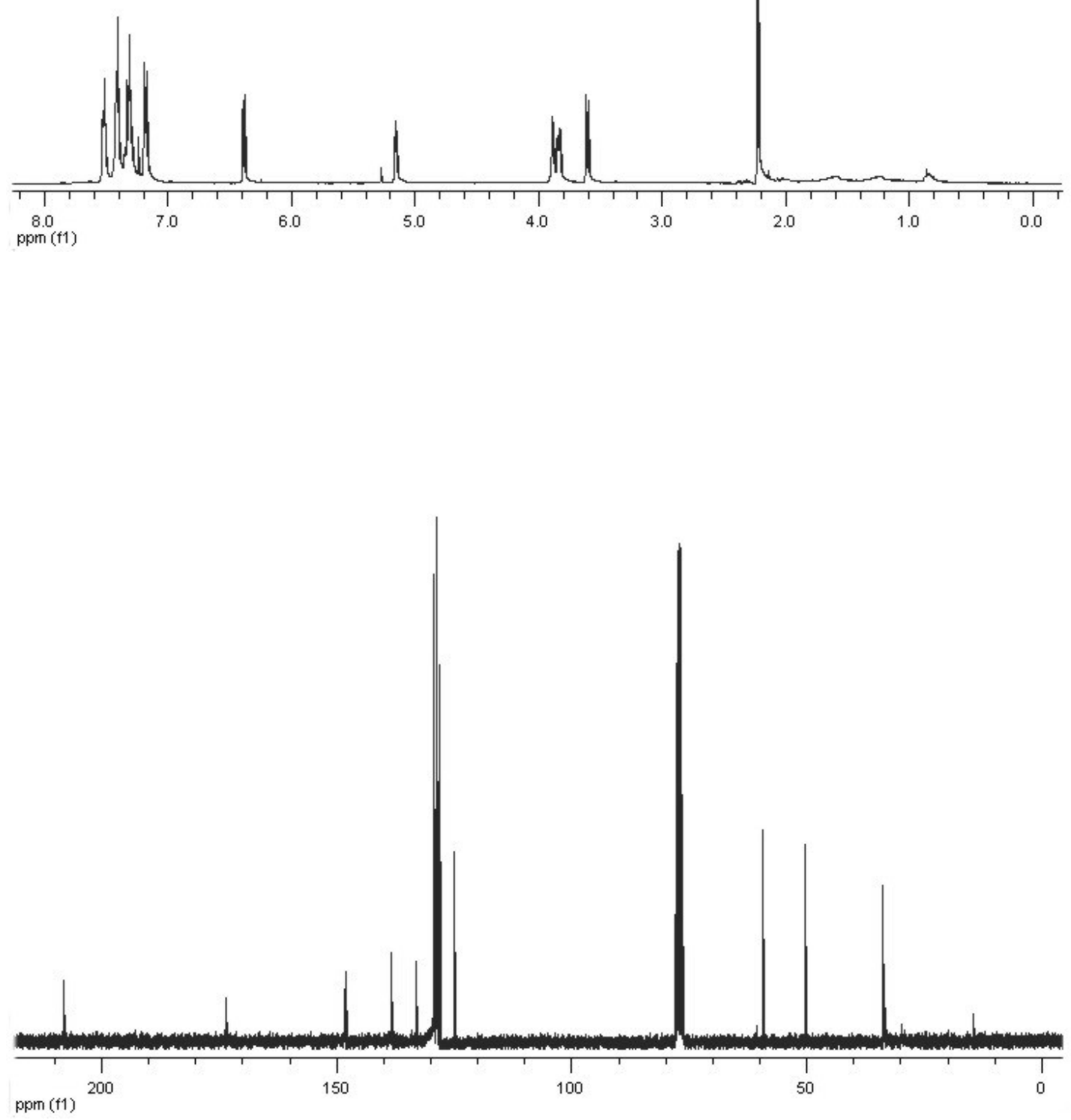

S 55 
HPLC, Chiralpak AD, hexanes: isopropanol $=90: 10,1.0 \mathrm{~mL} / \mathrm{min}, \lambda=220 \mathrm{~nm}$<smiles>CC(=O)CC1(O)C(c2ccccc2)=CC2OC(=O)C1O2</smiles>

racemic-7A'
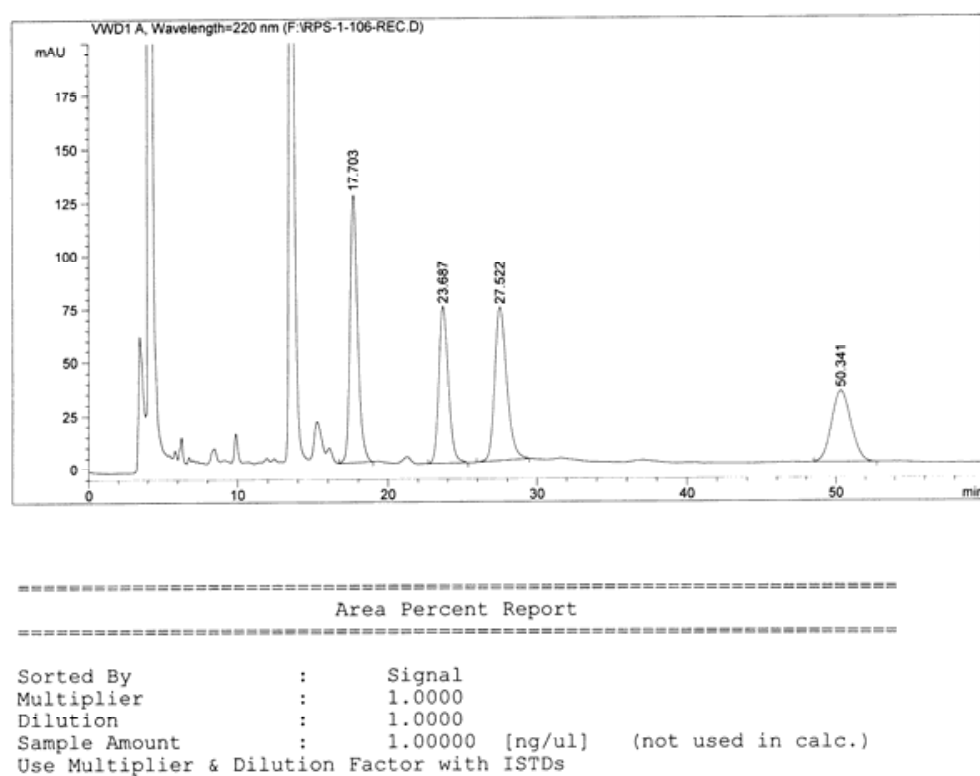

Signal 1: VWD1 A, Wavelength=220 nm

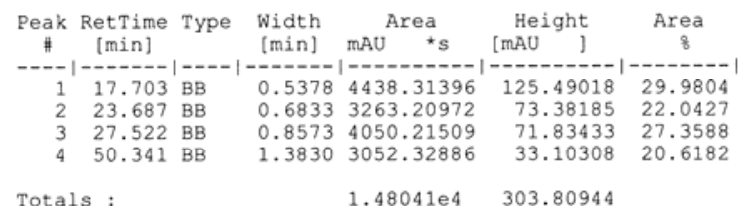

Totals :

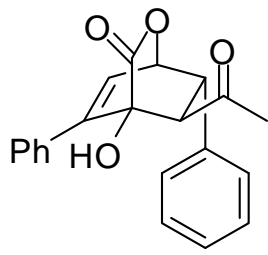

exo-7A', $96 \%$ ee obtained from QD-4 catalyzed reaction
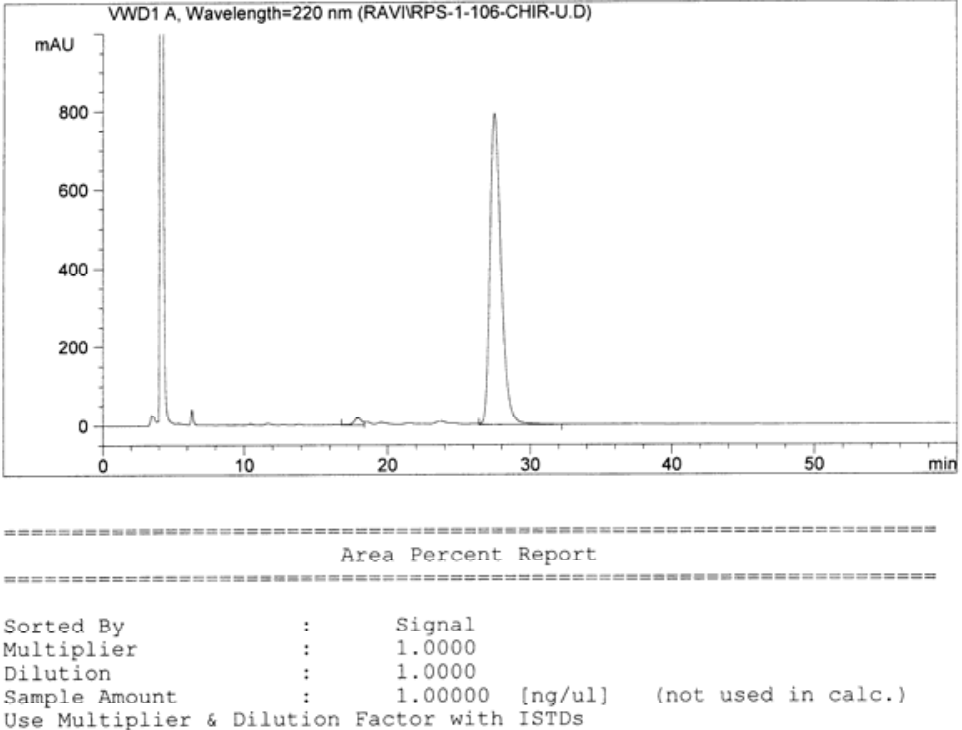

Signal 1: VWD1 A, Wavelengt $h=220 \mathrm{~nm}$

\begin{tabular}{|c|c|c|c|c|c|c|}
\hline $\begin{array}{c}\text { Peak } \\
\#\end{array}$ & $\begin{array}{c}\text { RetTime } \\
\text { [min] }\end{array}$ & Type & $\begin{array}{l}\text { Width } \\
\text { [min] }\end{array}$ & mAU ${ }^{\text {Area }}{ }^{*} \mathrm{~s}$ & $\begin{array}{c}\text { Height } \\
{[\mathrm{MAU}]}\end{array}$ & $\begin{array}{c}\text { Area } \\
8\end{array}$ \\
\hline $\begin{array}{l}1 \\
2\end{array}$ & $\begin{array}{l}17.933 \\
27.500\end{array}$ & $\begin{array}{l}\text { VV } \\
\text { VV }\end{array}$ & $\begin{array}{l}0.5705 \\
0.8318\end{array}$ & $\begin{array}{l}709.02301 \\
4.29977 \mathrm{e} 4\end{array}$ & $\begin{array}{r}18.69728 \\
791.47363\end{array}$ & $\begin{array}{r}1.6222 \\
98.3778\end{array}$ \\
\hline Total & : & & & $4.37067 \mathrm{e} 4$ & 810.1 & \\
\hline
\end{tabular}




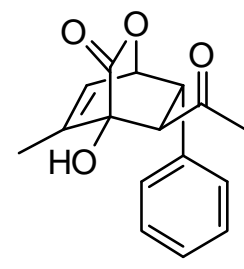

exo-7A"
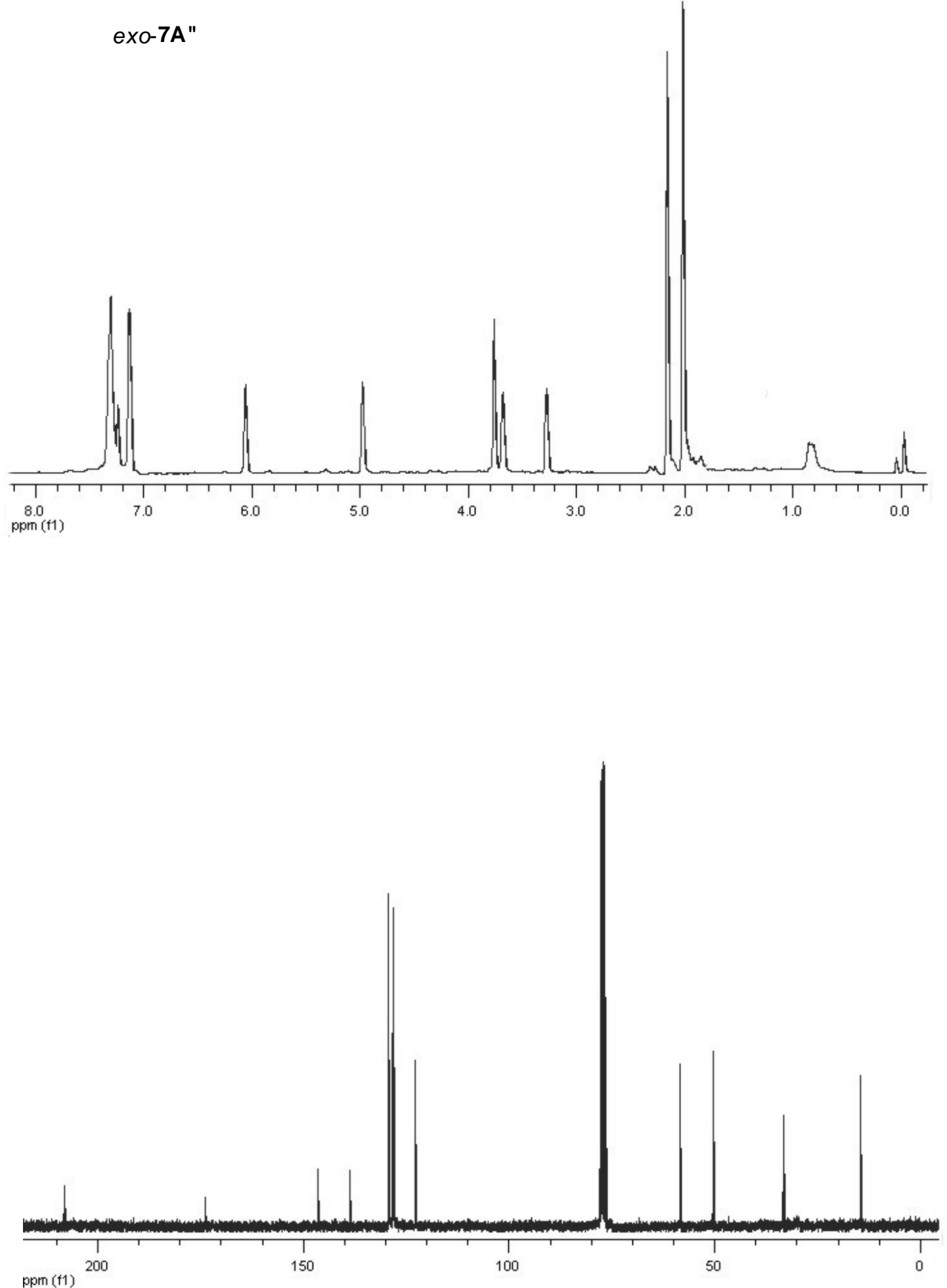

S 57 
HPLC, Chiralpak AD, hexanes: isopropanol $=95: 5,1.0 \mathrm{~mL} / \mathrm{min}, \lambda=220 \mathrm{~nm}$
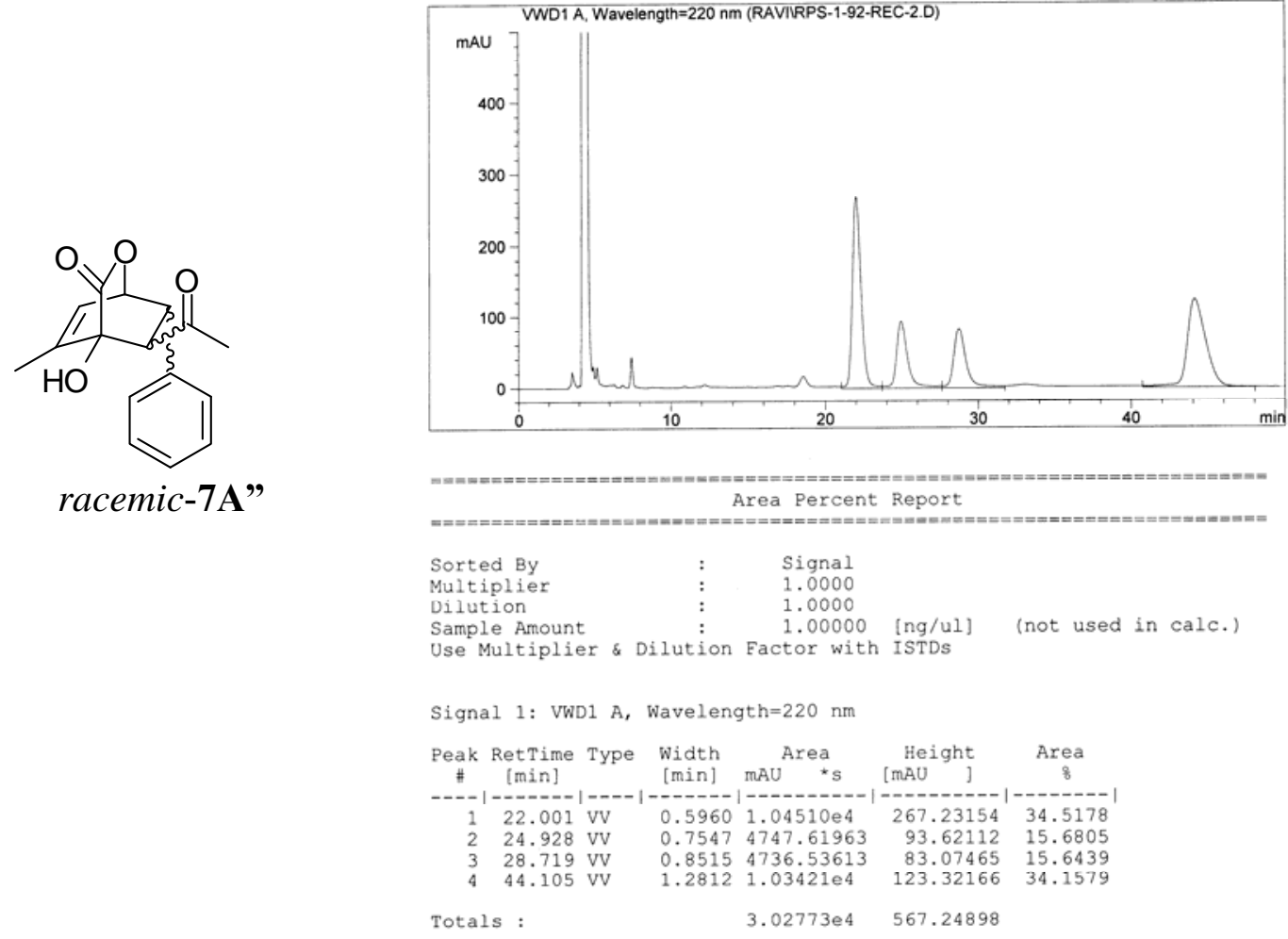

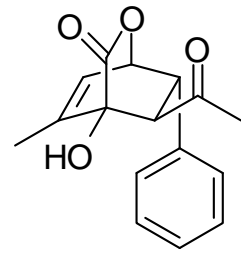

exo-7A", $96 \%$ ee obtained from QD-4 catalyzed reaction

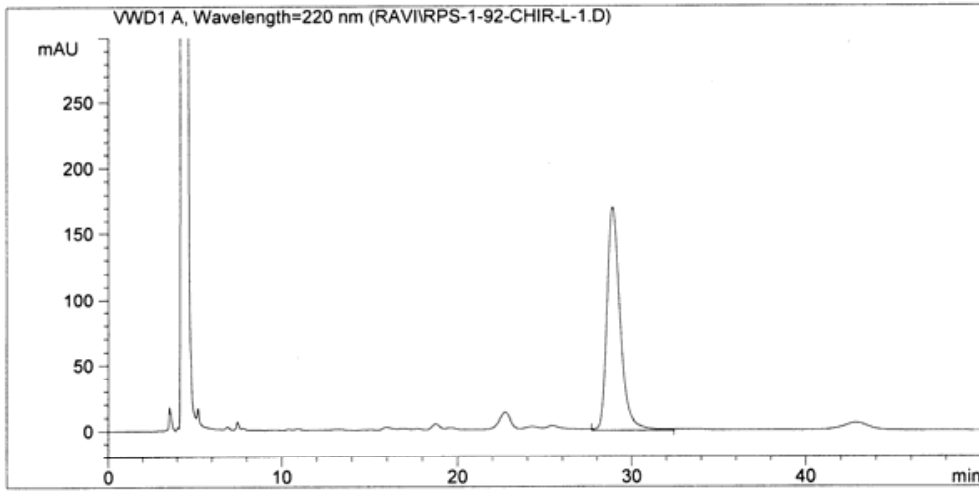

Area Percent Report

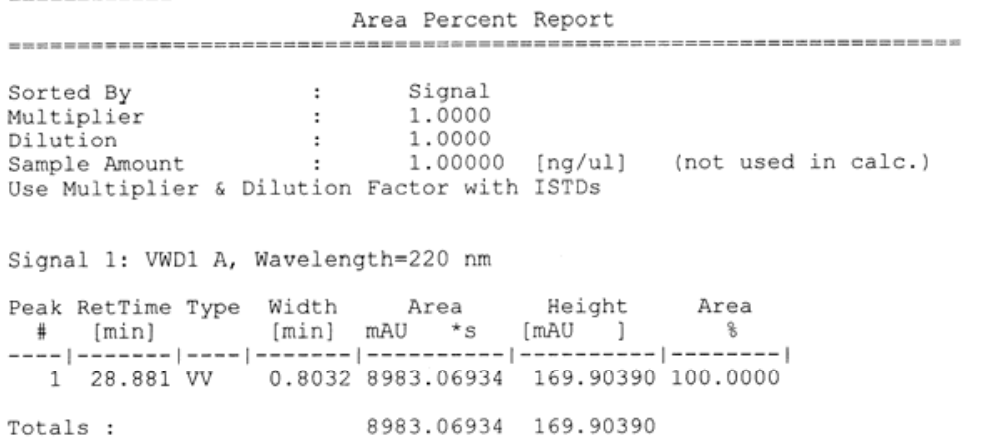



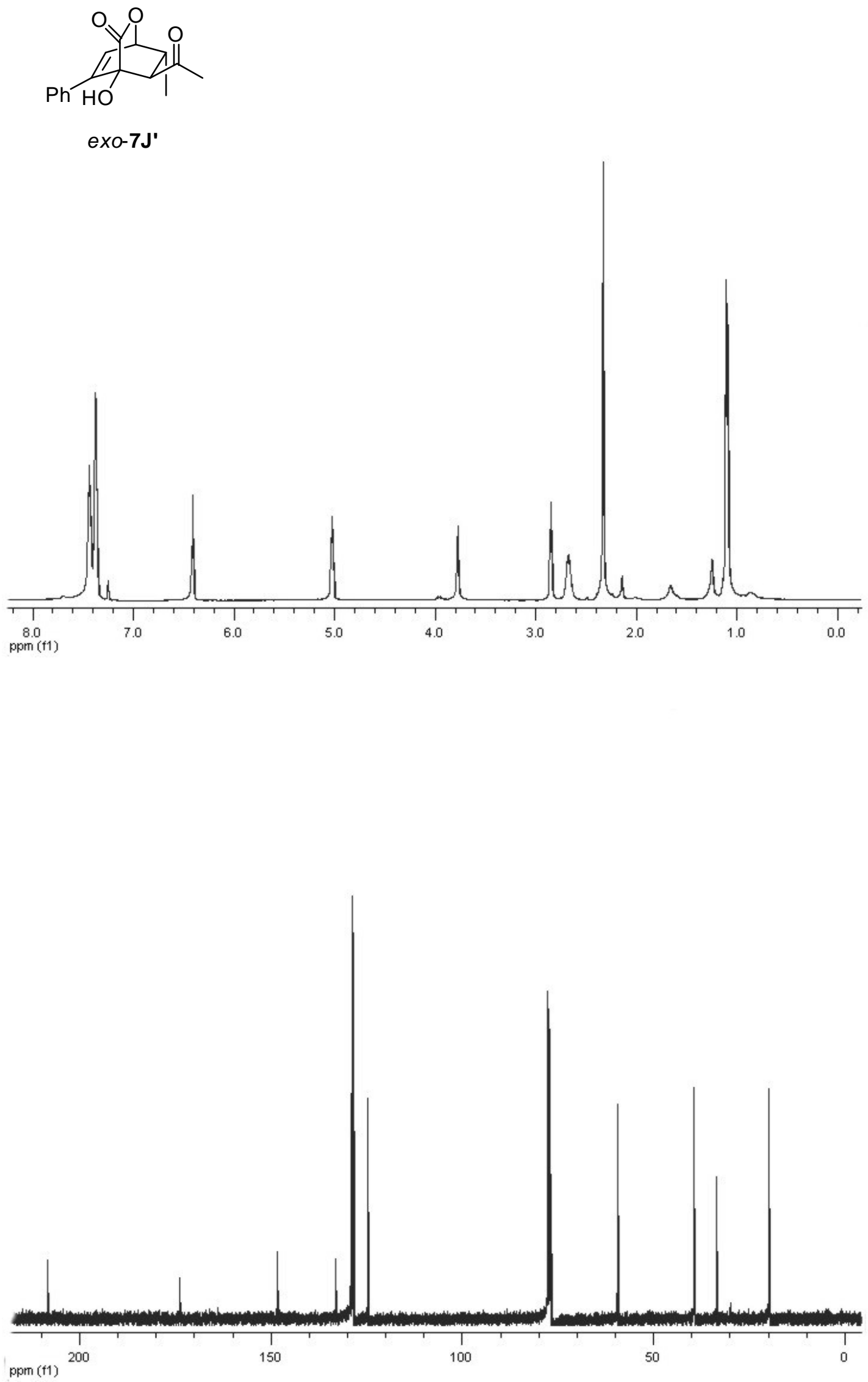

S 59 
HPLC, Chiralpak AD, hexanes: isopropanol $=90: 10,1.0 \mathrm{~mL} / \mathrm{min}, \lambda=220 \mathrm{~nm}$

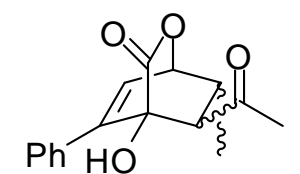

racemic-7J'
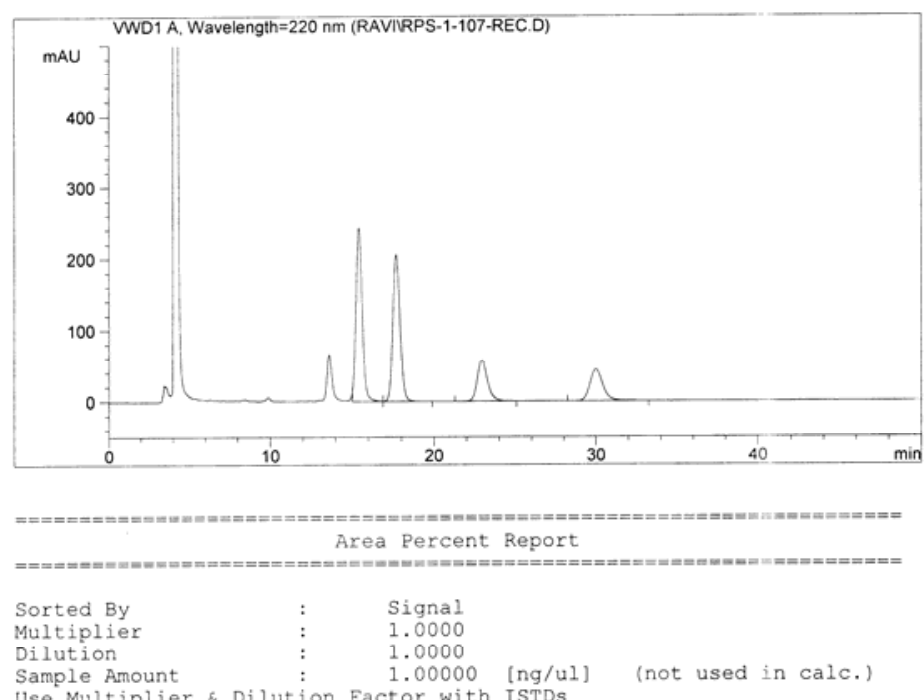

Use Multiplier \& Dilution Factor with ISTDs

Signal 1: VWD1 A, Wavelength $=220 \mathrm{~nm}$
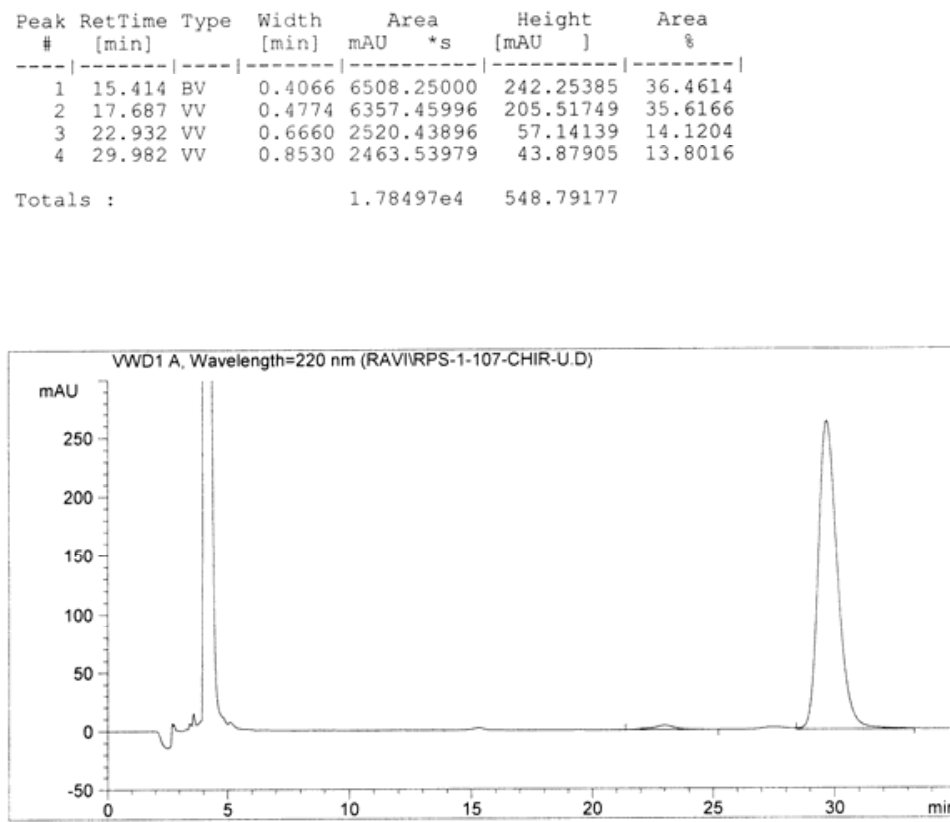

exo-7J', $96 \%$ ee obtained from QD-4 catalyzed reaction 

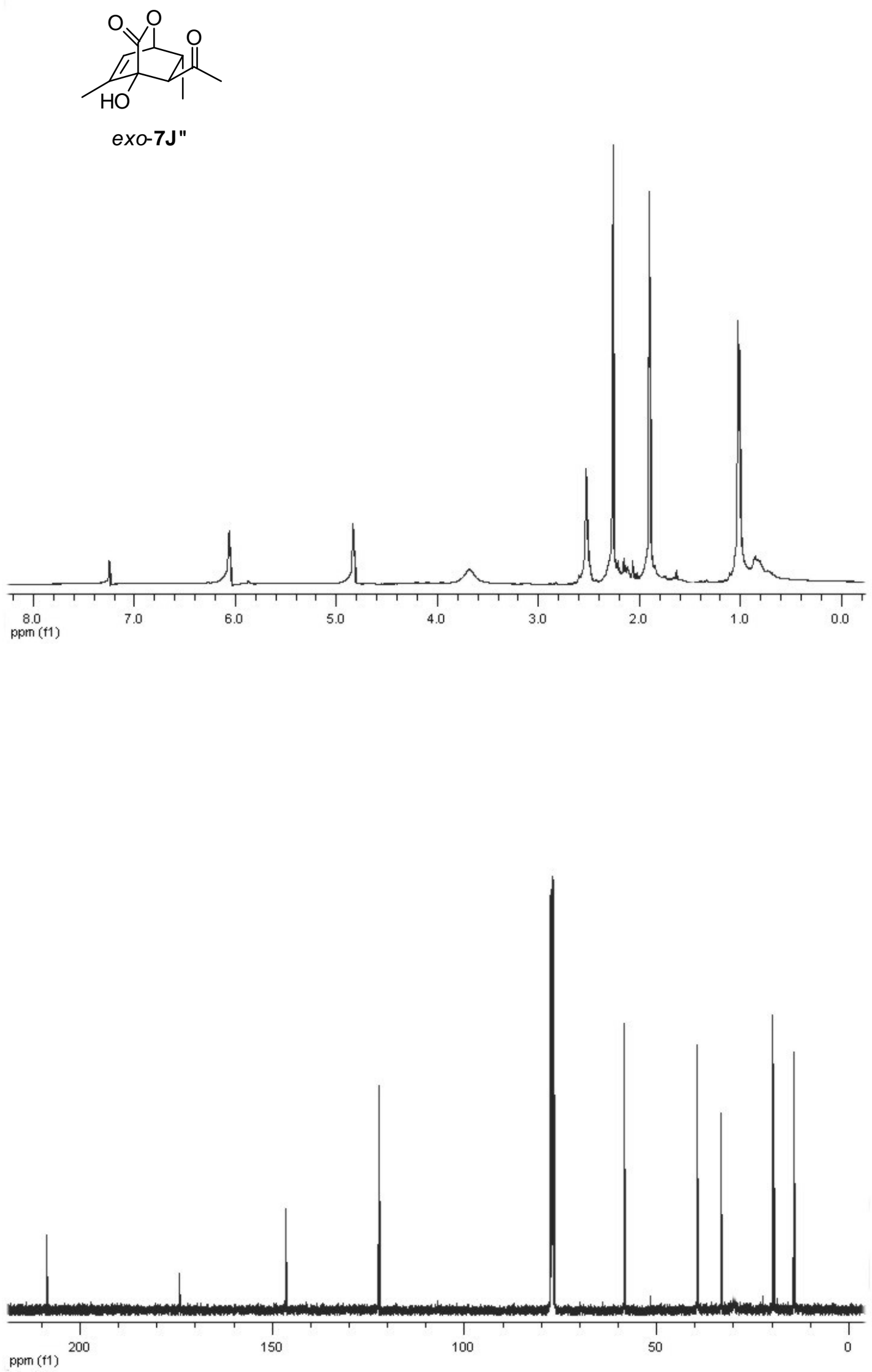

S 61 
HPLC, Chiralpak AD, hexanes: isopropanol $=95: 5,1.0 \mathrm{~mL} / \mathrm{min}, \lambda=215 \mathrm{~nm}$

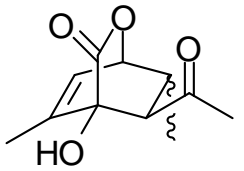

racemic-7
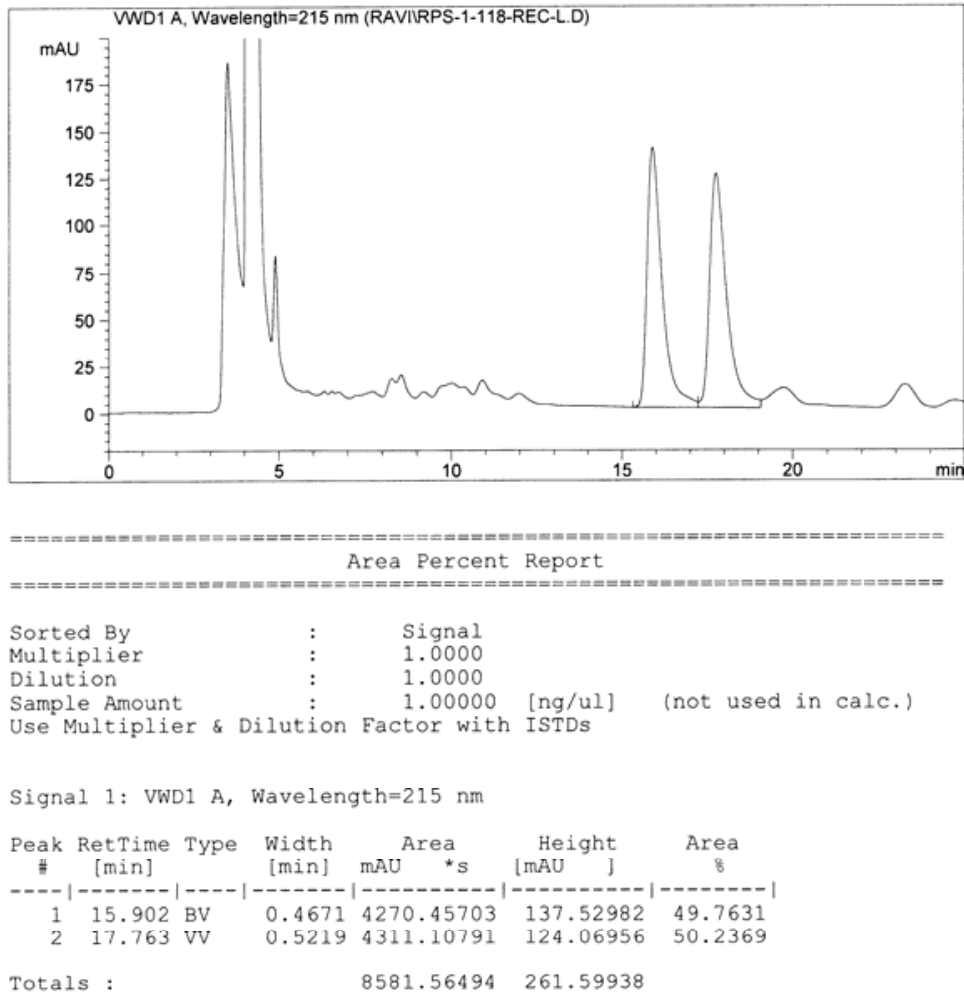

Totals :

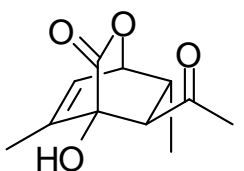

exo-7J", $98 \%$ ee obtained from QD-4 catalyzed reaction
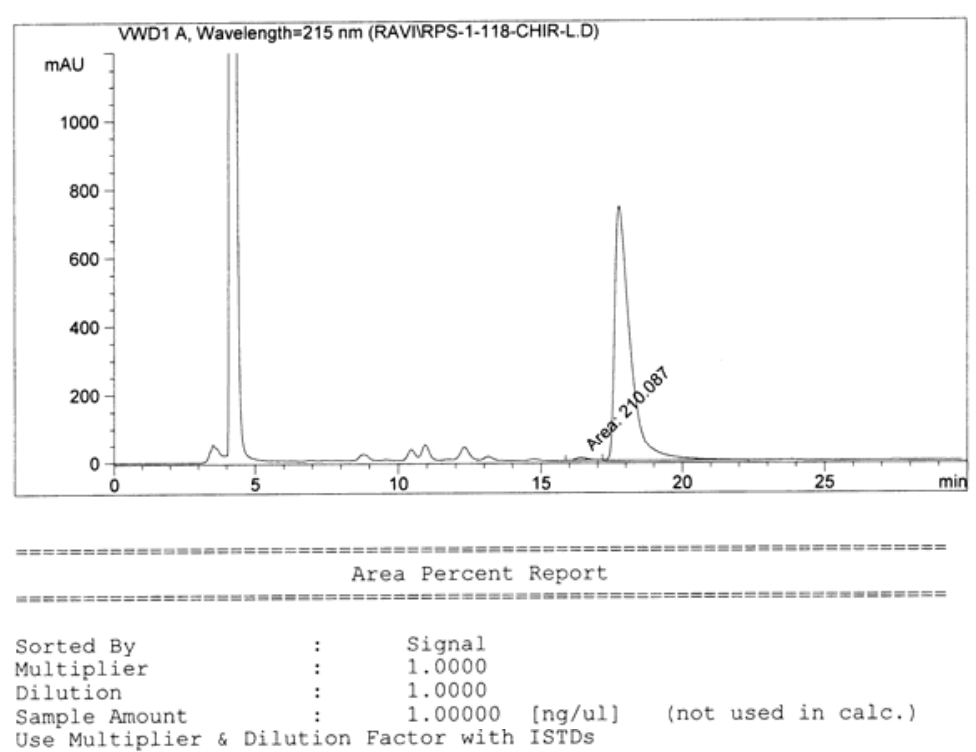

Signal 1: VWD1 $A$, Wavelength=215 nm

\begin{tabular}{|c|c|c|c|c|c|c|}
\hline Peak & RetTime & Type & Width & Area & Height & Area \\
\hline \# & [min] & & [min] & mAU & {$[\mathrm{mAU} \quad]$} & 8 \\
\hline $\begin{array}{l}1 \\
2\end{array}$ & $\begin{array}{l}16.417 \\
17.771\end{array}$ & $\begin{array}{l}\text { MM } \\
\text { VV }\end{array}$ & $\begin{array}{l}0.4450 \\
0.5610\end{array}$ & $\begin{array}{l}210.08725 \\
2.83451 \mathrm{e} 4\end{array}$ & $\begin{array}{r}7.86848 \\
744.01239\end{array}$ & $\begin{array}{r}0.7357 \\
99.2643\end{array}$ \\
\hline 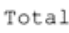 & & & & $2.85552 \mathrm{e} 4$ & 751.88087 & \\
\hline
\end{tabular}




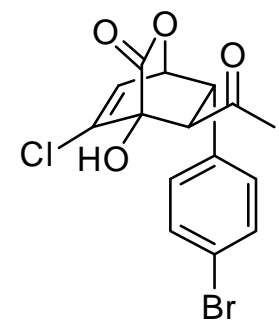

exo-7D'

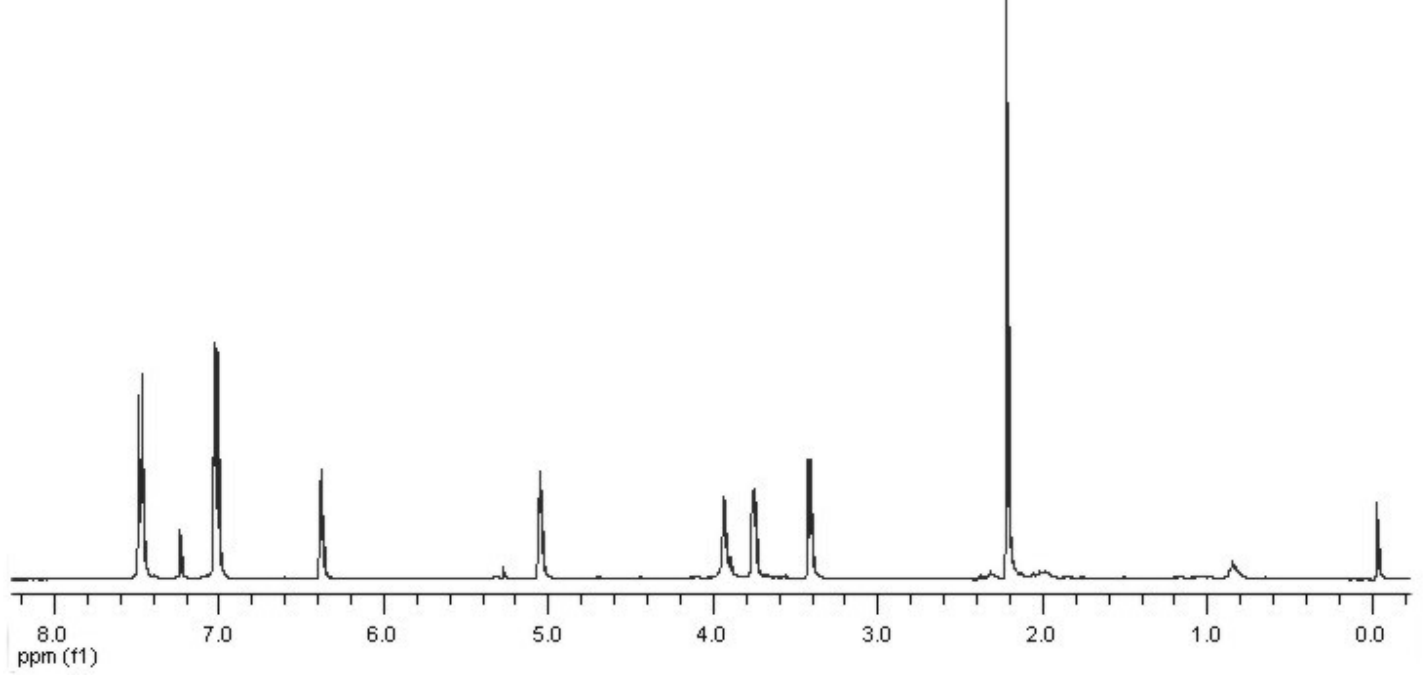

$13 \mathrm{c}$ onszave

Pulse Bequence: spoul

Solvent: CDC13

Nabient tesporature

nova-500 - gamblo"

prulae 53.1 dogroes

Aeq. timo 1.199.

844 repotitions

osazave c13, 100.5180130 neat

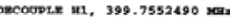

power $37 \mathrm{~dB}$

on during acquisition

GXRP-1 modulated

axp ppocegsro

tine broadening $1.0 \mathrm{~Hz}$

r size 65536

Total timo $1 \mathrm{hx}, 20 \mathrm{~min}, 40 \mathrm{sec}$

1.4.

200

180

160

140

120

100

80

60

40

20

ppm 
HPLC, Chiralcel OD, hexanes: isopropanol $=90: 10,1.0 \mathrm{~mL} / \mathrm{min}, \lambda=220 \mathrm{~nm}$.

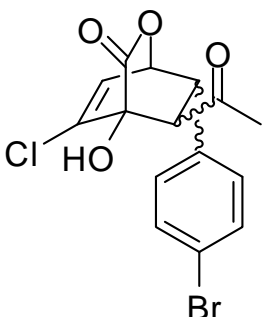

racemic-7D,

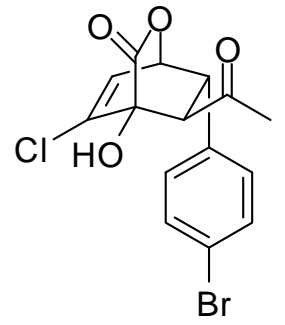

exo-7D', $90 \%$ ee obtained from QD-4 catalyzed reaction.
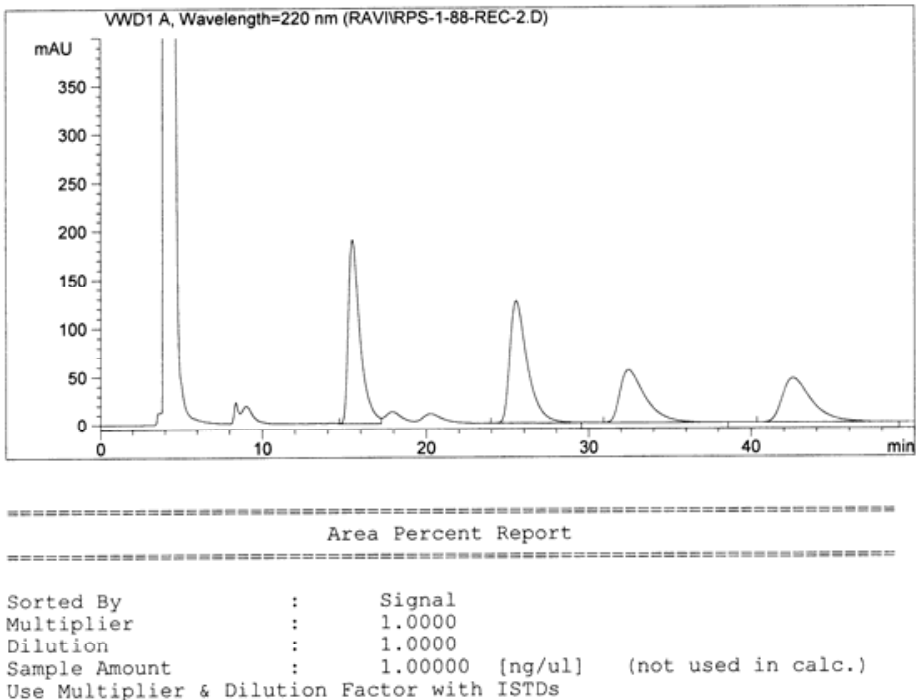

Use Multiplier \& Dilution Factor with ISTDs

Signal 1: VWD1 $A$, Wavelength $=220 \mathrm{~nm}$

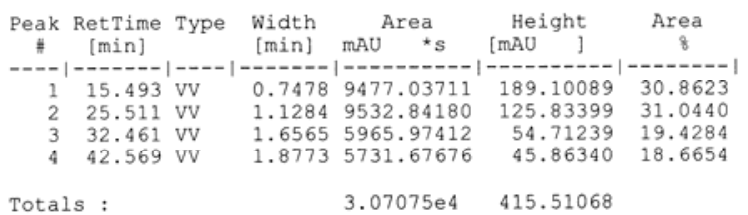

Totals : $\quad 3.07075 \mathrm{e} 4 \quad 415.51068$
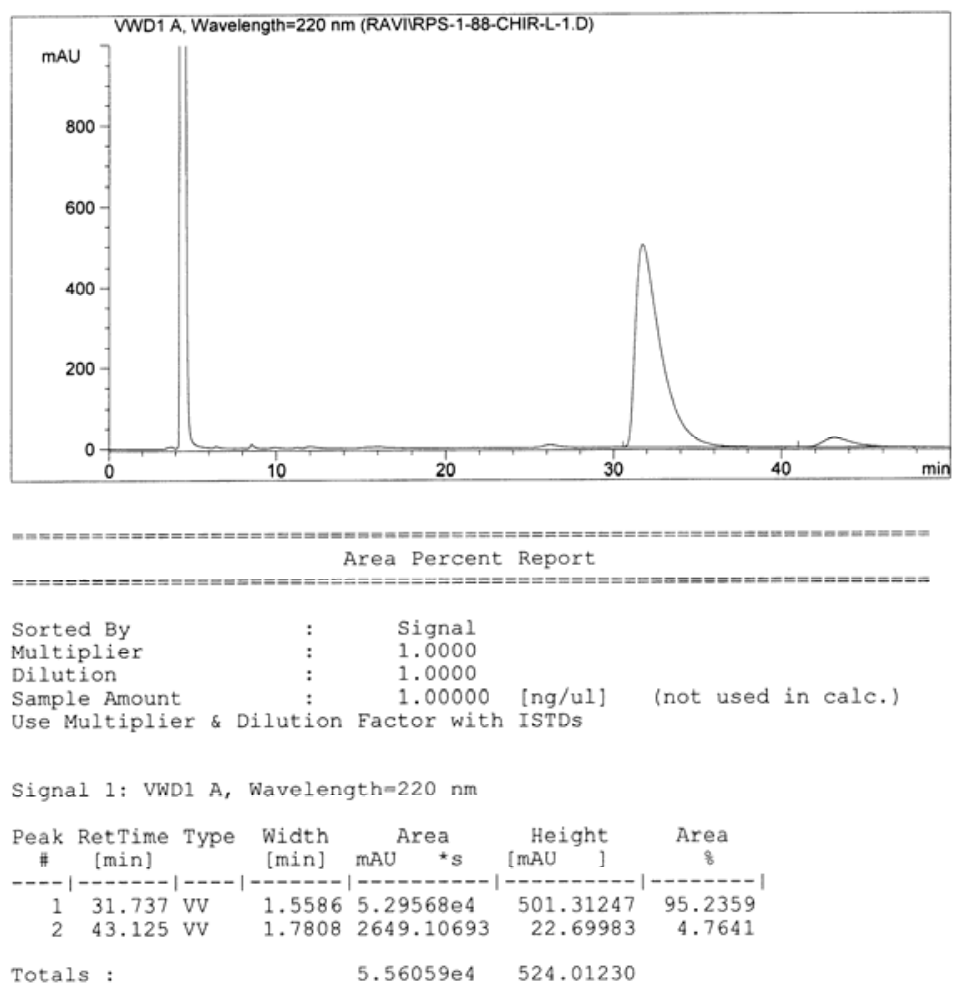
STMMDNRD 28 OBSERVE

pulae sequence: s2pul Solvent: CDC13 Amblent temperatur

IMOVA-400 "Tid" Pulee 46.1 degrees
Acq. time 1.638 sec Width $5000.0 \mathrm{~Hz}$

OBSzRVE $81,399.7532481$ Not

DATA PROCrssmo

Tratal timo $4 \mathrm{hr}, 34 \mathrm{~min}, 32 \mathrm{sec}$<smiles>CC(=O)C1=C(O)C=CC[C@H]1Pc1ccccc1</smiles>

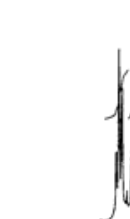

1
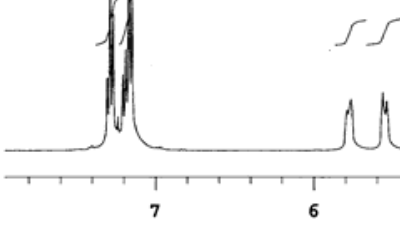

$13 \mathrm{C}$ OBservg

Pul ae sequence: s2pul

Solvent: $\mathrm{CDC}_{13}$

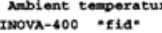

Pulse 53.1 degrees

width $25000.0 \mathrm{~Hz}$

404 repetitions

ODSzRVZ C13, 100.5180102 Mat

DECOUTLE H1, 399.7552472

Power 37 dB

on during acquisitio

off during delay

DMTA PROCESSING

Line broadening $1.0 \mathrm{~Hz}$

TT size 65536

Total time $335 \mathrm{hr}, 36 \mathrm{~min}, 45 \mathrm{sec}$

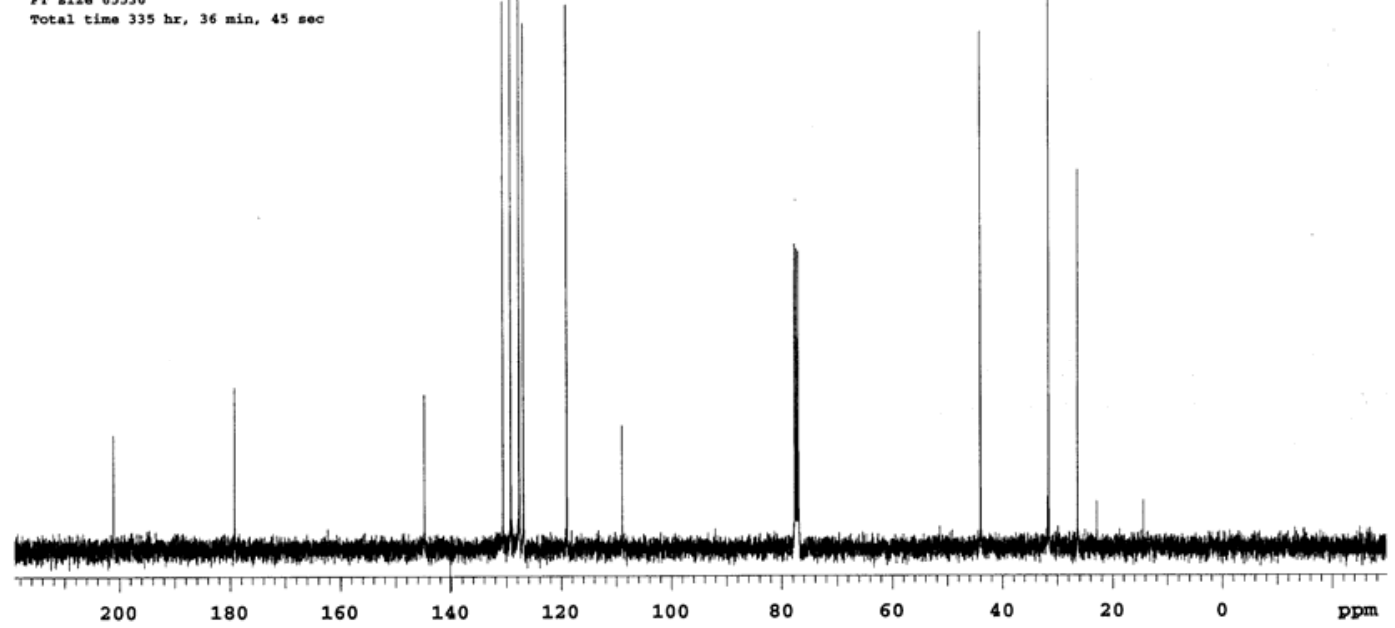


HPLC, Chiralcel OD, hexanes: isopropanol $=95: 5,1.0 \mathrm{~mL} / \mathrm{min}, \lambda=220 \mathrm{~nm}$.<smiles>CC(=O)C1=C(O)C=CC[C@H]1c1ccccc1</smiles>

racemic- $\mathbf{1 0}$
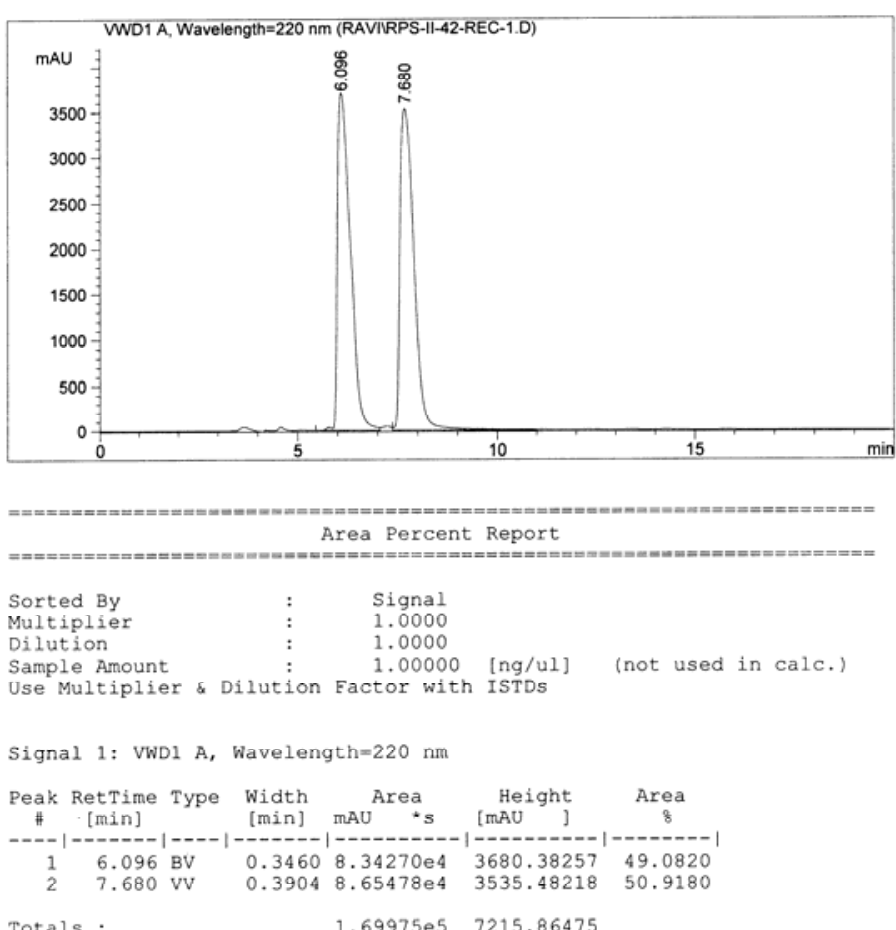

Totals :

$1.69975 \mathrm{e} 5 \quad 7215.86475$

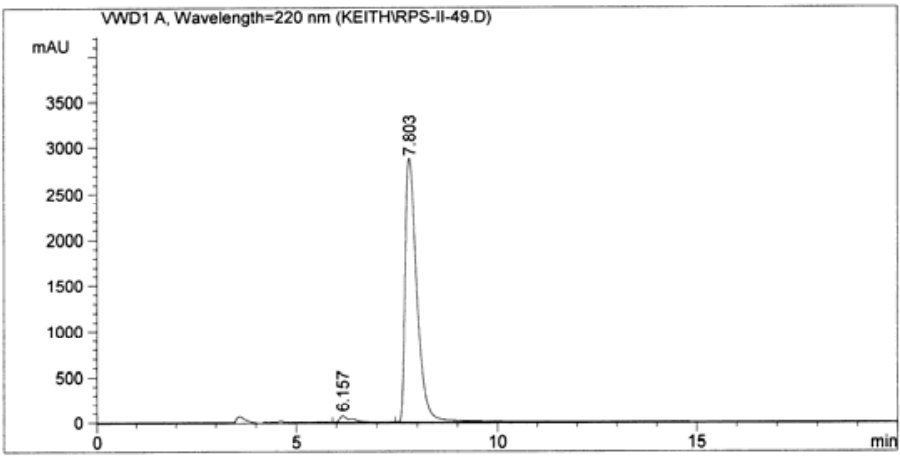

